\title{
Jean-Joseph MOISSET
}

Professeur, Département des fondements et pratiques en éducation, Université Laval

(1972)

\section{L’Alcan et la croissance économique du Saguenay -Lac-St-Jean}

\section{THÈSE \\ présentée à la Faculté de droit et des sciences économiques et sociales de l'Université de Fribourg (SUISSE)}

pour l'obtention du grade de docteur ès sciences économiques et sociales

Acceptée par la Faculté de droit et des sciences économiques et sociales, le 19 décembre 1972, sur la proposition de M. le professeur G. Gaudard (premier rapporteur) et de M. le Professeur J. Valarché (second rapporteur)

Un document produit en version numérique par Michel Fortin, bénévole, Adjoint à la mairie de Ville de Saguenay, province de Québec

Courriel: micfortin@videotron.ca ou micfor@ville.chicoutimi.qc.ca

Dans le cadre de: "Les classiques des sciences sociales" Une bibliothèque numérique fondée et dirigée par Jean-Marie Tremblay, professeur de sociologie au Cégep de Chicoutimi Site web: http://classiques.uqac.ca/

Une collection développée en collaboration avec la Bibliothèque Paul-Émile-Boulet de l'Université du Québec à Chicoutimi Site web: http://bibliotheque.uqac.ca/ 
Cette édition électronique a été réalisée par Michel Fortin, bénévole, adjoint à la mairie de Ville de Saguenay, à partir de :

Jean-Joseph Moisset

\section{L'ALCAN ET LA CROISSANCE ÉCONOMIQUE DU SAGUENAY-LAC-ST-JEAN (QUÉBEC)}

THÈSE présentée à la Faculté de droit et des sciences économiques et sociales de l'Université de Fribourg (SUISSE) par Jean J. MOISSET (Meaux, HAITI)

pour l'obtention du grade de docteur ès sciences économiques et sociales. Acceptée par la Faculté de droit et des sciences économiques et sociales, le 19 décembre 1972, sur la proposition de M. le professeur G. Gaudard (premier rapporteur) et de M. le Professeur J. Valarché (second rapporteur)

M. Jean-Joseph Moisset, professeur au Département des fondements et pratiques en éducation, de l'Université Laval, nous a accordé son autorisation de diffuser sa thèse de doctorat le 6 septembre 2006 dans Les Classiques des sciences sociales.

9f Courriel : Jean-Joseph.Moisset@fse.ulaval.ca

Polices de caractères utilisée :

Pour le texte: Times New Roman, 12 points.

Pour les citations : Times New Roman, 12 points.

Pour les notes de bas de page : Times New Roman, 10 points.

Édition électronique réalisée avec le traitement de textes Microsoft Word 2004 pour Macintosh.

Mise en page sur papier format : LETTRE (US letter), 8.5'’ x 11’’)

Édition numérique réalisée le 25 février 2007 à Chicoutimi, Ville de Saguenay, province de Québec, Canada. 


\title{
L'ALCAN ET LA CROISSANCE ÉCONOMIQUE DU SAGUENAY-LAC-ST-JEAN (QUÉBEC)
}

\author{
THÈSE \\ présentée à la Faculté de droit \\ et des sciences économiques et sociales \\ de l'Université de Fribourg (SUISSE) \\ par
}

Jean J. MOISSET

(Meaux, HAITI)

pour l'obtention du grade

de docteur

ès sciences économiques et sociales

Acceptée par la Faculté de droit et des sciences économiques et sociales, le 19 décembre 1972, sur la proposition de M. le professeur G. Gaudard (premier rapporteur) et de

M. le Professeur J. Valarché (second rapporteur) 


\section{Table des matières}

$\underline{\text { Avant-propos }}$

Liste des principales abréviations utilisées

LISTE DES CARTES ET FIGURES

LISTE DES TABLEAUX

INTRODUCTION

\section{PREMIÈRE PARTIE \\ Le Saguenay-Lac-St-Jean avant l'implantation de l'Alcan}

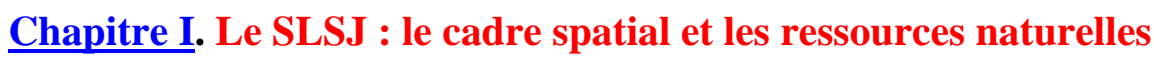

Section 1. Le cadre spatial

Section 2. Potentialité du Saguenay_Lac-St-Jean

2.1. Le complexe relief - climat - sols - eaux

2.2. Les ressources énergétiques

2.3. Les ressources minérales

Chapitre II. Le SLSJ : les éléments humains

Section 1. La démographie régionale

1.1. Les chiffres de population

1.2. La structure de la population

\section{Section 2. La main-d'œuvre régionale}

2.1. Le problème quantitatif et structurel

2.2. Le problème qualitatif 


\section{DEUXIÈME PARTIE \\ L'Alcan et l'évolution de l'économie du SLSJ}

Chapitre III. L'implantation de l'Alcan dans le SLSJ

Section 1. La métallurgie de l'aluminium

1.1. Le procédé Bayer

1.2. Le procédé électrolyse

Section 2. Les facteurs de la localisation de l'Alcan dans le SLSJ

2.1. Les facteurs défavorables

2.2. Les facteurs favorables

\section{Section 3. L'implantation}

3.1. Les préalables

3.2. Les installations

Chapitre IV. La triple nature de l'Alcan

Section 1. Alcan, grande unité inter-territoriale

1.1. Les origines de l'Alcan

1.2. L'activité industrielle de l'Alcan

Section 2. Alcan, entreprise nationale

2.1. Le contexte canadien de l'Alcan

2.2. L'expansion de l'Alcan et la position du Canada sur le marché de l'aluminium

Section 3. Alcan, entreprise saguenayenne

3.1. Développement hydroélectrique du SLSJ

3.2. L'industrie saguenayenne d'aluminium

3.3. L'Alcan et les transports au SLSJ 
Chapitre V. Le repérage des indicateurs de la croissance économique du SLSJ

Section 1. La production régionale et évolution

1.1. Le secteur primaire

1.2. Le secteur secondaire

1.3. Le secteur tertiaire

Section 2. Le revenu et le niveau de vie

2.1. Le revenu : évaluation et évolution

2.2. La structure du revenu

2.3. Population, main-d'œuvre et revenu

\section{TROISIÈME PARTIE}

Le rôle moteur de l'Alcan

\section{Chapitre VI. Les effets de l'Alcan sur le SLSJ}

Section 1. Les effets directs

1.1. L'Alcan et les investissements régionaux

1.2. L'Alcan et l'emploi régional

1.3. L’Alcan et les revenus régionaux

\section{Section 2. Les effets indirects et induits}

2.1. La polarisation technique

2.2. La polarisation financière et la polarisation par les revenus

2.3. La polarisation psychologique

2.4. La polarisation spatiale 
Chapitre VII. Les limites du processus de développement du SLSJ à partir de l’Alcan

Section 1. Les obstacles provenant du milieu

1.1. Les structures économiques

1.2. Le problème humain

Section 2. Les obstacles provenant de l'Alcan

2.1. La théorie du comportement de la GUI

2.2. L'Alcan-GUI et l'économie du SLSJ

CONCLUSION GÉNÉRALE

BILBIOGRAPHIE 


\section{AVANT-PROPOS}

$\underline{\text { Retour à la table des matières }}$

Il y aura bientôt vingt ans, le Professeur François Perroux lançait lidée de "pôles de croissance ». La faveur que cette nouvelle idée a connue auprès des milieux académiques et les responsables économiques non seulement ne s'est pas démentie mais encore s'est consolidée avec le temps. Elle s'est enrichie progressivement pour devenir la théorie du développement régional polarisé, aujourd'hui partie intégrante de la science économique.

Le but de cette étude est d'examiner, à la lumière de cette théorie, l'évolution d'une région, le Saguenay_Lac-St-Jean au Québec, face à un complexe industriel d'aluminium, celui de l'Alcan, et de montrer indirectement la marge nécessaire entre la théorie et la réalité, entre l'outil d'analyse et l'instrument de politique économique.

Nous tenons à exprimer d'abord notre profonde reconnaissance à l'égard du Professeur G. Gaudard pour sa direction attentive et bienveillante.

Nous remercions également le Service des Relations Publiques de l'Alcan, à Arvida, pour sa précieuse collaboration, ainsi que tous les organismes et bibliothèques qui ont accepté aimablement de mettre leurs documents à notre disposition.

Nous remercions enfin tous ceux qui, de près ou de loin, nous ont permis de poursuivre nos études et de mener à terme cette tâche.

Jean J. Moisset 


\section{LISTE DES PRINCIPALES ABRÉVIATIONS UTILISÉES}

Retour à la table des matières

ALCAN : $\quad$ Aluminium du Canada Limitée et ses filiales

BFS : $\quad$ Bureau Fédéral de la Statistique

BIT : $\quad$ Bureau International du Travail

BRE : $\quad$ Bureau de Recherche Économique

CÉR : $\quad$ Conseil Économique Régional

COÉQ: $\quad$ Conseil d'Orientation Économique du Québec

CSN : $\quad$ Confédération des Syndicats Nationaux

GUI : Grande Unité Inter-territoriale

I.SEA : Institut de Science Économique Appliquée

MIC : $\quad$ Ministère de l'Industrie et du Commerce

OPDQ : $\quad$ Office de Planification et de Développement du Québec

Ry. : $\quad$ Railway

SLSJ : $\quad$ Saguenay-Lac-St-Jean 


\section{LISTE DES CARTES ET FIGURES}

$\underline{\text { Retour à la table des matières }}$

1. Le Saguenay_Lac-St-Jean dans le Québec

2. Le réseau hydrographique du Saguenay_-Lac-St-Jean

3. Évolution de la population du Saguenay_Lac-St-Jean, 1857-1966

4. Pyramide des âges comparées de la province de Québec, 1881-1951

5. Production d'aluminium, Canada, 1910-1968

6. Centrales hydroélectriques du Saguenay_Lac-St-Jean, 1968

7. Les agglomérations du Saguenay_Lac-St-Jean : pôles et zones d'influence

\section{LISTE DES TABLEAUX}

1. Canada : régions économiques

2. Les forces hydrauliques du Saguenay-Lac-St-Jean,1928

3. $\quad$ Saguenay_Lac-St-Jean, population, 1851-1966

4. Structure par figes de la population du Québec et du Saguenay_Lac-St-Jean, 1961-1966

5. $\quad$ Répartition de la population canadienne selon les occupations, 1881-1951

6. $\quad$ S.LSJ : main-d'œuvre selon l'occupation, 1941

7. $\quad$ SLSJ : population agglomérée, 1931

8. Chronologie de la mise en place des chemins de fer du Saguenay_Lac-St-Jean

9. $\quad$ Les usines d'alumine de 1'Alcan, Monde, 1972

10. Les usines d'aluminium de 1'Alcan, Monde, 1972

11. Alcan : répartition des ventes d'aluminium

12. Alcan : bilan consolidé au 31 décembre 1971

13. Alcan : répartition géographique du personnel

14. Alcan : rétrospective décennale, 1962-1971

15. Alcan : filiales et participations

16. Alcan : activité industrielle au Canada, 1971

17. Production d'aluminium, Monde et Canada, 1900-1971

18. Le développement hydroélectrique au S.L.S.J

19. Centrales hydroélectriques du Saguenay-Lac-St-Jean appartenant à l'Alcan

20. Alcan : main-d’œuvre et salaires au S.L.S.J.,1967 
21. Port-Alfred : trafic global et bauxite,1952-1965

22. Port-Alfred : structure du trafic, 1965

23. Produit intérieur brut par tète, Québec et régions, 1951-1966

24. Valeur ajoutée brute par secteurs, Saguenay—Lac-St-Jean, 1951-1966

25. SLSJ : état de l'agriculture, 1921

26. SLSJ : production et consommation d'aliments

27. SLSJ : Principaux outillages agricoles

28. SLSJ : valeur ajoutée par principaux types d'industries, 1961-1965

29. SLSJ : nombre et poids relatif des établissements manufacturiers, 1965

30. SLSJ : développement industriel, 1932-1943

31. SLSJ : le commerce de détail, 1931-1941

32. Revenu disponible par tète, Québec et régions 1951-1966

33. SLSJ : structure du revenu disponible,1951-1966

34. Zone urbaine de Chicoutimi et Province de Québec : salaires hebdomadaires moyens

35. SLSJ : répartition du personnel d'Alcan

36. Ville d'Arvida : relevé des taxes payées par la Compagnie Alcan, 1963-1972

37. Saguenay - Lac-St-Jean : industries de services et industries utilisant l'aluminium

38. Saguenay—Lac-St-Jean : poids relatif des industries de produits métalliques

39. Saguenay—Lac-St-Jean : production et consommation de viandes, 1965

40. Saguenay_Lac-St-Jean : dépendance industrielle envers Arvida

41. Saguenay_Lac-St-Jean : caractéristiques des centres urbains

42. Saguenay - Lac-St-Jean : secteur manufacturier, poids relatif des groupes majeurs, 1965

43. SLSJ : Salaire annuel moyen et valeur ajoutée par employé dans le secteur manufacturier, 1965

44. Les industries manufacturières du Québec par régions administratives, 1964 


\section{ALCAN 1 ET LA CROISSANCE ÉCONOMIQUE DU SAGUENAY-LAC ST-JEAN (QUÉBEC)}

\section{INTRODUCTION}

$\underline{\text { Retour à la table des matières }}$

«Le problème du développement économique a toujours été l'un des pôles fondamentaux d'intérêt de l'économie politique » 2. Déjà Adam Smith, développant ses « Recherches sur la nature et les causes de la richesse des nations », concluait à « une progression historique généralisée des nations, liée à l'extension des marchés » et à son corollaire, «la division du travail ». Faisant preuve de perspicacité dans l'observation et de lucidité dans le raisonnement, il constatait cependant « une variation considérable de la marche du progrès de l'opulence entre les peuples et les siècles », qu'il expliquait par le fait que « la ville ne tire pas toujours la totalité de ses subsistances de la campagne qui l'avoisine, ni même du territoire auquel elle appartient, mais qu'elle les tire souvent de campagnes fort éloignées » 3.

Il y a là une intuition géniale à laquelle on ne se réfère certes plus aujourd'hui, mais qui est sous-jacente à l'hypothèse de base de toutes les théories modernes de la croissance économique, à savoir que «l'inégalité de croissance entre les nations et les régions accompagne inévitablement la croissance elle-même » 4.

1 ALCAN : désignation abrégé propre à l’Aluminium du Canada Limitée et à ses filiales.

2 P. A. Baran, Économie politique de la croissance, Maspero, Paris, 1967, p. 4.

3 A. Smith, Recherches sur la nature et les causes de la richesse des nations, Paris, 1843, p. 740.

4 A. Hirschman, Stratégie du développement économique, traduction de F. Pons, Les Éditions Ouvrières, Paris, 1964, p. 210. 
L'analyse, relativement récente, de la croissance comme « processus de polarisation, puis de diffusion» procède également de cette idée de base. Partant de l'observation de l'inégale distribution de la croissance, le Professeur François Perroux en posa ainsi les bases : «le fait grossier, mais solide, est celui-ci : la croissance n'apparaît pas partout à la fois; elle se manifeste en des points ou pôles de croissance, avec des intensités variables ; elle se répand par divers canaux et avec des effets terminaux variables pour l'ensemble de l'économie » 5.

Quel que soit le terrain sur lequel on se place, de la géographie ou de la théorie économique, on peut très difficilement contester l'idée que des phénomènes d'inégalité et de déséquilibre sont inhérents à la croissance. En fait, souligne J. Freyssinet, « personne ne soutient plus aujourd'hui la possibilité d'un développement également distribué entre toutes les unités économiques et qui reproduise, de période en période, les conditions de l'équilibre marginaliste » 6.

Axiomatique, la notion de pôle de croissance eût été vaine, ne fût-ce le caractère fonctionnel que voulut lui donner son auteur. En effet, écrit Perroux, « scruter cette modalité de la croissance (la polarisation), c'est rendre explicite et scientifiquement maniable une vue présentée déjà dans plusieurs élaborations théoriques, imposée par l'observation des pays à croissance retardée, apparente dans la politique des États modernes » 7.

Du fait de son caractère d'outil d'analyse, d'instrument de politique économique, le concept de pôle de croissance, souvent mal compris, si l'on en croit Jean Paelinck, a déjà fait l'objet de nombreuses études. D'après cet auteur, «on l'a confondu avec les notions d'industrie clef, d'industrie de base, d'ensemble industriel,... de point de croissance, dont l'action serait spontanée et géographiquement concentrée. » J. Paelinck s'élève contre ces interprétations qu'il estime mauvaises, car « constitue un pôle de croissance une industrie qui, par les flux de produits et de revenus qu'elle engendre, conditionne le développement et la croissance d'industries

5 F. Perroux, Note sur la notion de pôle de croissance, Cahiers de l'I.S.É.A., p.143.

6 J. Freyssinet, Le concept de sous-développement, Mouton, Paris, 1966, p. 276.

7 F. Perroux, L'économie du XXe siècle, PUF, Paris, 1964, pp. 143-144. 
techniquement liées à elle, détermine la prospérité du secteur tertiaire par les revenus qu'elle engendre et produit une croissance du revenu régional grâce à la concentration de nouvelles activités dans une zone donnée, moyennant la perspective de pouvoir disposer de certains facteurs de production $» 8$.

On savait bien déjà avec le Professeur Perroux que le pôle de croissance est une unité motrice dans un milieu donné 9. Mais, la définition de Paelinck, plus exhaustive, permet de dégager d'emblée les quatre types fondamentaux de modalités d'action du pôle de croissance, à savoir : la polarisation technique, la polarisation des revenus, la polarisation psychologique et la polarisation spatiale.

À côté des analyses théoriques dont le but a été d'apporter plus de systématisation et de rigueur au concept de " pôle de croissance », beaucoup d'autres, plus modestement, se sont penchées sur les applications du développement régional polarisé. Du reste, ce deuxième aspect de la théorie du pôle de croissance est complémentaire du premier et d'une grande utilité. Il est vrai, comme le fait ressortir Bridgman, que «la valeur d'une conception, comme son sens profond, doivent être trouvés en observant ce qu'un homme accomplit (ou peut accomplir) avec elle et non ce qu'il dit sur elle » 10. Tenant ou s'opposant à la théorie de la croissance polarisée, on a ainsi senti la nécessité de la confronter avec la réalité. Le champ offert par les pays sous-développés et les régions à croissance retardée des pays industrialisés s'y prêtait d'ailleurs fort bien.

Les démarches effectuées dans cette perspective peuvent être classées en deux catégories : d'une part, l'application des idées de polarisation à l'élaboration de plans de développement régional ; d'autre part, l'analyse des problèmes soulevés par l'implantation d'une industrie ou d'un complexe industriel dans une région, à la lumière de la théorie du développement régional polarisé.

8 J. Paelinck, La théorie du développement régional polarisé, Cahiers de l'ISEA, mars 1965, pp. 11-12.

9 F. Perroux, La firme motrice dans la région et la région motrice, Cahiers de l’ISEA, mars 1961, p. 301 ss.

10 W. Bridgman, «The Logic of Modern Physics », dans J. Boudeville, Les espaces économiques, Collection QSJ, p.18. 
La présente étude se rangerait dans cette dernière catégorie. Son titre, à cet effet, est significatif : " l'Alcan et la croissance économique du Saguenay-Lac-St-Jean ». Quel rôle l'Alcan, grande société de production d'aluminium, a-t-il joué dans la croissance économique de cette région de la Province de Québec ? Quel rôle aurait-il pu y jouer ou peut-il encore y jouer ? Telles sont les questions essentielles auxquelles ce travail tâchera d'apporter une réponse.

Puisque, par définition, « tout développement est un processus impliquant deux aspects fondamentaux, le temps et l'espace » 11, cette étude se réfèrera à un cadre temporel plus ou moins précis, se situant entre la période d'implantation de l'Alcan dans la région et les années les plus récentes. Quant au cadre spatial, une première démarche sera de le définir.

Cette recherche revêtira un certain caractère partiel, car elle ne procède pas d'une enquête systématique sur le terrain, ce qui aurait exigé des moyens considérables. Or, dans ce domaine, des matériaux statistiques nécessaires font parfois défaut et ceux qui sont disponibles ne sont pas tous homogènes.

Cela conduit à ne pas envisager, pour cette analyse, l'élaboration d'un modèle de type inputoutput, dont on connaît cependant la valeur quand il s'agit de dégager les caractères structurels d'une économie et les effets quantifiables d'une industrie sur cette économie. Mais, comme l'a fait justement remarquer F. Perroux, «nous sommes souvent contraints de restreindre nos analyses à partir de la documentation au lieu d'étendre la documentation à la demande de l'analyse » 12.

Cette étude comportera trois parties. Dans la première, le Saguenay_Lac-St-Jean sera défini en tant que région et on en verra les données caractéristiques avant l'implantation de la Société Alcan. La deuxième partie sera consacrée à une présentation des différentes dimensions de l'Alcan et essaiera de saisir simultanément l'évolution de quelques variables stratégiques de

11 T. Hermansen, Growth and Growth centers in National and Regional Development, A Synthetical Approach, part 1, pp. 1-2.

12 F. Perroux, La firme motrice dans la région et la région motrice, op. cit., p. 294. 
l'économie régionale. Enfin, dans la dernière partie, on tentera, après avoir mis en relief les principaux effets de l'Alcan sur le Saguenay_Lac-St-Jean, de caractériser, à la lumière de la théorie du développement régional polarisé, le rôle de cette société dans la dynamique de l'économie saguenayenne. 


\section{PREMIÈRE PARTIE \\ Le Saguenay-Lac-St-Jean avant l'implantation d'Alcan}

$\underline{\text { Retour à la table des matières }}$

Il est presque banal de dire que l'activité économique en général et la croissance en particulier se déroulent dans un espace donné et que toute analyse dans ce domaine doit être précédée d'une définition du concept de « région » et d'une délimitation de la région concrète à laquelle on se réfère. En lui-même, le concept de région n'est pas très aisé à manier, tant il est vrai que les différentes disciplines, notamment la géographie et l'économie, par leurs querelles, relevant souvent de l'esprit de clocher, ont jeté plus de confusion que de lumière dans les esprits.

À l'évidence, la région du géographe, « territoire quelconque possédant une certaine originalité, une certaine spécificité »13, ne recouvre pas forcément celle de l'économiste, s'il est admis que « les espaces économiques se définissent par les relations économiques existant entre des éléments économiques » 14.

13 P. Claval, Régions, nations et grands espaces, Éd. M. Th. Génin, Paris, 1968 p. 299 et ss.

14 F. Perroux, L'économie du XXe siècle, op. cit., p. 127. 
Entre les deux définitions précédentes de la région, l'une concrète l'autre abstraite mais les deux trop vagues, il y a encore place pour de solides discussions que les économistes, d'ailleurs entre eux, n'ont pas cessé d'entretenir avec chaleur. Certes, on est loin de l'époque où l'on pouvait affirmer que la région est «un concept intellectuel, une entité pour la pensée par le choix de certaines caractéristiques importantes pour un problème régional » 15.

Incontestablement, l'apport du Professeur Perroux, «analysant la région économique en termes de région homogène, région polarisée et région plan ", a contribué à combler ce maquis foisonnant où les esprits les plus éclairés se perdaient 16. Cependant, il faut reconnaître que la région économique, définie comme « celle qui forme un tout absolument indépendant et séparé du reste du monde et où, par conséquent, il n'existe aucun mouvement de produits, de services, de maind’œuvre avec l'extérieur » 17 est idéale et n'existe pas. Ou si elle a une existence, elle coïncide avec le globe, ce qui revient au même. Dans cette perspective, toute analyse économique régionale se situe au départ dans une problématique difficile. Lors même que pour lever ce handicap majeur on définit la région comme « lieu d'échange de biens et services dont l'intensité interne est supérieure en chaque point à l'intensité externe »18, on est bien obligé de recourir, au départ tout au moins, à un espace banal, entendu dans le sens perrousien de territoire concret et défini. Auguste Lösch avait pressenti ces difficultés, qui écrivait : « une région économique claire est un heureux accident, plutôt qu'une subdivision naturelle des États »19.

De fait, dans les analyses concrètes d'économie régionale, « on en est venu dans bien des cas... à se rallier à des régions qui coïncident avec les entités administratives traditionnelles mais qui par ailleurs possèdent une certaine cohésion, celle-ci s'appliquant tant à la région

15 E.J. Preston, F.J. Clarence, dans Pierre Moran, L'analyse spatiale en science économique, Cujas, Paris, 1966, p. 7.

16 J. Boudeville, Les espaces économiques, op. cit., p.7.

17 A. Raynauld, Croissance et structure économique de la Province de Québec, Québec, 1961, p. 33.

18 J. Boudeville, L'économie régionale, espace opérationnel, Cahiers de l’ISEA, série L, no 3, p. 7.

19 A. Lösch, The Nature of Economic Regions, dans P. Moran, op. cit., p. 36. 
homogène qu'à la région polarisée »20. Ainsi en sera-t-il de cette étude, dont le cadre sera la région administrative du Saguenay_Lac-St-Jean. Il est cependant évident que la croissance économique, phénomène à faces multiples, ne saurait être réduite à un cadre spatial strictement limité. J. Paelinck a fort justement noté que «la croissance ne s'opère pas isolément au bénéfice d'une région déterminée »21. Dans la mesure où les données statistiques régionales sont en général réunies par unités administratives, le territoire choisi pour ce travail écartera les inconvénients qui découlent, selon P. Moran, de la nature même de la région, « ensemble ouvert, sans monnaie propre et présupposant une organisation territoriale plus vaste disposant du monopole de la contrainte publique et des attributs de la souveraineté »22.

20 R. Parenteau, Les problèmes du développement régional dans un État fédératif : l'expérience canadienne, Rapport au Congrès des Économistes de langue française, mars 1963, p.163.

21 J. Paelink, L. Davin, L. Degeer, Dynamique économique de la région liégeoise, Éd. de l’ASLB, le Grand Liège, 1959, p. 14.

22 P.Moran, op. cit., p. 44. 
Première partie : Le Saguenay_Lac-St-Jean

avant l'implantation de l'Alcan

\section{Chapitre I}

\section{Le Saguenay-Lac-St-Jean: le cadre spatial et les éléments naturels}

\section{Section 1 : Le cadre spatial}

$\underline{\text { Retour à la table des matières }}$

Il n'y a personne, pas plus aujourd'hui qu'hier, pour nier l'importance de la géographie d'un territoire en regard de son développement économique. Cette vérité prend un caractère particulièrement aigu en ce qui concerne l'économie canadienne en général et l'économie québécoise en particulier. Cette idée a été mise en relief avec une nette vigueur par R. D. Howland qui écrit :

Si l'on peut dire que le Canada représente une victoire politique sur la géographie, cette victoire n'a pas éliminé la géographie en tant qu'élément de l'économie. Le régionalisme est en fait un trait essentiel de l'économie canadienne, un phénomène qui remonte jusqu'à la constitution du pays, laquelle du reste subit l'influence des intérêts régionaux et des caractéristiques spéciales23.

De fait, le Canada, État fédératif, constitué de dix Provinces plus ou moins autonomes, est loin de former un tout homogène. Partant de cette observation, divers auteurs ont essayé de subdiviser le Canada en régions économiques. Pour sa part, Howland, laissant pour un traitement

23 R. D. Howland, Certains aspects régionaux du développement économique du Canada, Commission Royale d'enquête, Ottawa, 1957, p. 1. 
spécial le Nord du pays (soit le Yukon et les Territoires du Nord-Ouest, plus de 40\% de tout le sol canadien), distingue, en se basant sur la similarité de leurs structures socioéconomiques, cinq grandes régions : les Provinces de l'Atlantique, le Québec, l'Ontario, les Prairies et la ColombieBritannique.

On remarquera au passage que certains auteurs ne distinguent que quatre régions au Canada, considérant que les provinces de l'Ontario et du Québec ont des structures économiques suffisamment semblables pour être réunies en une région économique24.

Tableau 1

Canada : régions économiques

$\underline{\text { Retour à la table des matières }}$

\begin{tabular}{|c|c|c|c|}
\hline Région, province, territoire & $\begin{array}{c}\text { Superficie en } \\
\text { milles carrés }\end{array}$ & Les terres & $\begin{array}{c}\text { \% de la } \\
\text { superficie totale }\end{array}$ \\
\hline Provinces de l'Atlantique & 206601 & 198394 & 5,6 \\
\hline - Terre-neuve & 155364 & 147994 & 4,2 \\
\hline - Île-du-Prince-Édouard & 2184 & 2184 & -- \\
\hline - Nouvelle-Écosse & 21068 & 20743 & 0,6 \\
\hline - Nouveau-Brunswick & 27985 & 27473 & 0,8 \\
\hline Québec & 594860 & 523860 & 14,7 \\
\hline Ontario & 412582 & 333835 & 9,4 \\
\hline Les Prairies & 753497 & 688705 & 19,4 \\
\hline - Manitoba & 246512 & 219723 & 6,2 \\
\hline - Saskatchewan & 251700 & 220182 & 6,1 \\
\hline - Alberta & 255285 & 248800 & 7,0 \\
\hline Colombie-Britanique & 366255 & 359279 & 10,1 \\
\hline Yukon et Territoire du Nord-Ouest & 1511979 & 1458784 & 40,9 \\
\hline Total Canada & $\mathbf{3 8 4 5} \mathbf{4 7 4}$ & $\mathbf{3 5 6 2 8 5 7}$ & $\mathbf{1 0 0 , 0}$ \\
\hline
\end{tabular}

Source : Annuaire du Canada, BFS, (Bureau Fédéral de la Statistique), cité par R.D. Howland.

24 Caves, Holton, The Canadian Economy, Prospect and Retrospect, Harvard University Press, 1959, dans R. Parenteau, op. cit., p.180. 
Comme il ressort du tableau précédent, le Québec, à lui seul, couvre une superficie de près de 600000 milles carrés, soit environ trois fois celle de la République française. Cependant, l'immensité de son étendue ne doit en rien faire préjuger de son poids économique réel. En effet, la population de ce vaste territoire s'élève aujourd'hui approximativement à six millions d'habitants. Comparée avec celle de plus de cinquante millions de la France, la population du Québec est numériquement faible. Et même, en rapprochant ces deux grandeurs, population et superficie, on peut a priori imaginer des difficultés d'ordre économique, pour cette province, explicables par son état de souspeuplement relatif. Exprimé de façon différente, ce point de vue a été retenu par R. Parenteau qui cite «l'énormité des distances à parcourir» au nombre des «causes principales du retard de croissance de certaines régions du Canada »25. L'on comprend dès lors qu'il est non seulement possible mais encore nécessaire de porter le regard sur des ensembles moins vastes. La Province du Québec se partage en dix régions administratives (carte 1, page suivante), au nombre desquelles se trouve le Saguenay—Lac-St-Jean.

25 R. Parenteau, idem., p.198. 


\section{Carte 1}

\section{Le Saguenay—Lac-St-Jean dans la province de Québec}

$\underline{\text { Retour à la table des matières }}$

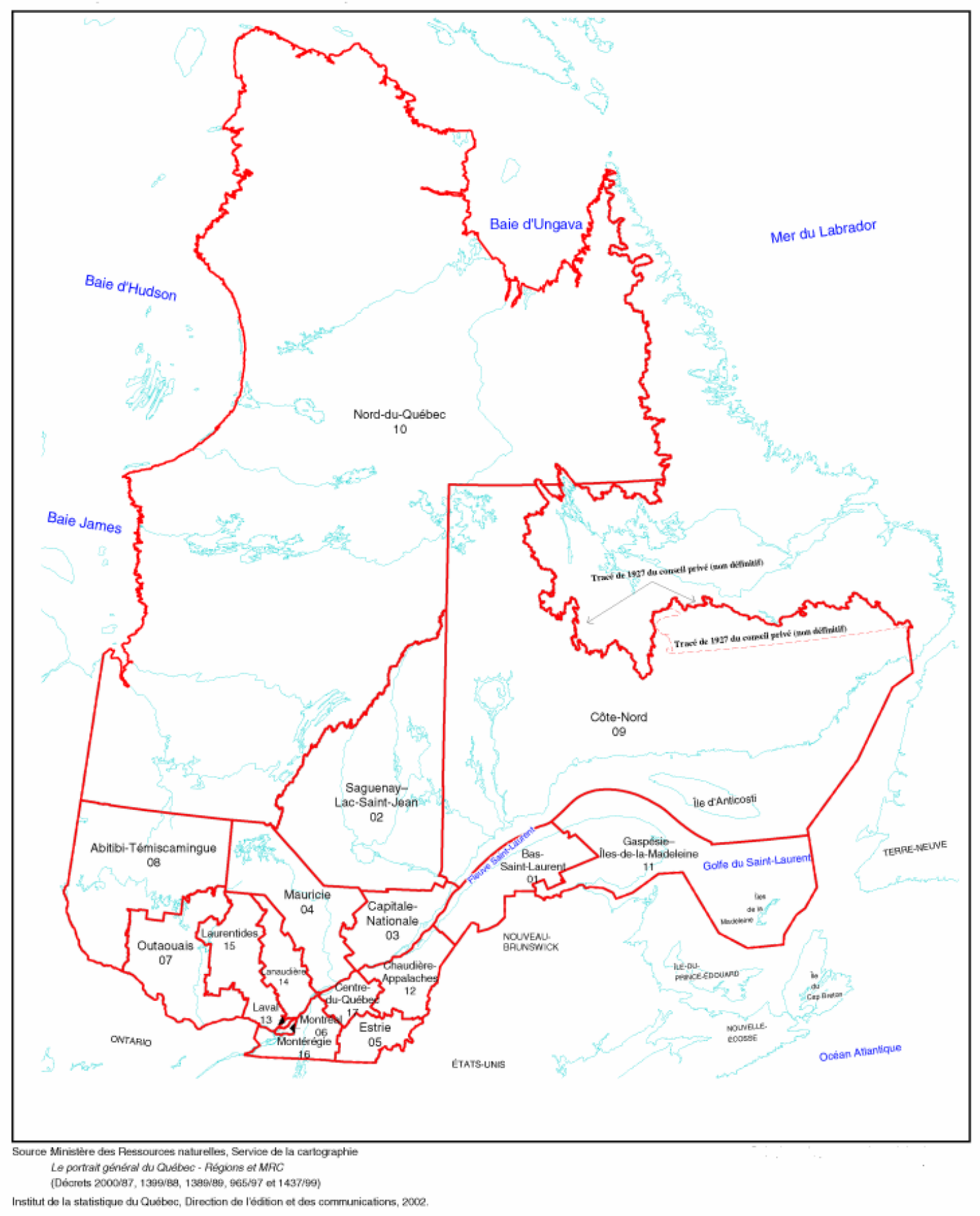


Cette région naturelle située au Nord-Ouest de l'estuaire du fleuve St-Laurent, sur la bordure orientale du Bouclier Canadien, coïncide de façon approximative avec le bassin de drainage du Lac St-Jean et la section supérieure de la vallée de la rivière du Saguenay, d'où son nom. Elle couvre une superficie de 41000 milles carrés, soit plus de deux fois celle de la Suisse. « Les arpenteurs qui ont établi le cadastre de la Province du Québec ont partagé la région en trois comtés municipaux : Chicoutimi (17 800 milles carrés), le Lac St-Jean Est (905 milles carrés) et le Lac St-Jean Ouest (22 818 milles carrés), dont la frontière orientale est le $70^{\circ}$ degré de longitude ouest, la frontière méridionale le $48^{\circ}$ degré de latitude nord et la frontière occidentale, en majeure partie, la ligne de partage des eaux entre le bassin du St-Laurent et celui des baies James et d'Hudson »26. À ces trois comtés primitifs devait s'ajouter en 1951 la sous-région de Chibougamau-Chapais, pour donner en définitive la région administrative du Saguenay-Lac-St-Jean. Dans la mesure où « l'environnement est le support de l'habitat et des activités, la condition du développement social et économique »27, il n'est peut-être pas inutile de faire une brève présentation des caractéristiques physiques de la région28. L'ensemble du Saguenay-Lac-St-Jean se compose de trois zones nettement distinctes : la cuvette du Lac St-Jean, le Fjord Saguenay et le Haut-Saguenay.

\subsection{La cuvette du Lac St-Jean}

$\underline{\text { Retour à la table des matières }}$

«La présence de larges étendues de faible altitude (environ 200 mètres), pourvues par l'alluvionnement de la mer Champlain de vastes terrasses fertiles et entourées pourtant de rudes plateaux élevés » forme le profil d'ensemble de cette première zone. On notera que la partie du

26 F.A. Angers et autres, Perspectives économiques de la région du SLSJ, 1952, p.4, Rapport préparé pour le Conseil d'Orientation Économique du SLSJ.

27 P.-Y. Pépin, Le Royaume du Saguenay en 1968, Ministère de l'Expansion Économique Régionale, Ottawa, 1969, p.19.

28 R. Blanchard, L'Est du Canada français, tome 2, Beauchemin, Montréal, pp. 7 à 155. 
Nord-Ouest, « la plus éloignée de l'estuaire » est dotée de « la plus grande étendue de terres basses et exploitables $» 29$.

\subsection{Le Fjord Saguenay}

$\underline{\text { Retour à la table des matières }}$

«Le glacier local du Lac St-Jean, alimenté par un vaste bassin versant, est responsable de la création de la rivière Saguenay, l'ombilic du lac. Plus loin, à l'aval, concentrant sa masse en un seul lit étroit entre les plateaux, le glacier y a foré une belle vallée en auge aboutissant au St-Laurent et dont la mer a fait un fjord. C'est une voie de passage naturelle, désenclavant le lac St-Jean à l'intérieur. »

\subsection{Le Haut-Saguenay}

« Le travail du glacier, aux prises avec le bloc du verrou de Kénogami, n'a d'ailleurs pas suffi à briser toute la résistance de l'obstacle. Les encoches qu'il a pratiquées de chaque côté, si elles ont eu l'immense intérêt de permettre à la mer d'envahir la région du Lac St-Jean, n'ont pu être poussées assez profondément pour aménager une pente régulière... Là est la région des paysages difficiles que l'homme saura utiliser pour de splendides réalisations industrielles » 30.

De cette description sommaire du paysage physique de la région, on peut déjà retenir, sans pour autant faire du fatalisme géographique en économie, que le Saguenay-Lac-St-Jean se compose de deux zones marquées du point de vue de l'activité économique, l'une à vocation agricole - celle du Lac St-Jean - et l'autre à vocation industrielle - celle du Saguenay. Mais, auparavant, il faut voir de plus près les ressources naturelles.

29 R.Blanchard, idem, p.59.

30 R. Blanchard, ibidem, pp. 59-60. 


\section{Section 2 : Potentialités du Saguenay—Lac-St-Jean}

$\underline{\text { Retour à la table des matières }}$

«L'analyse des activités créatrices de biens et services implique celle des facteurs dont elles résultent directement, les facteurs naturels... ou indirectement, les facteurs humains » 31. En ce qui concerne les potentialités physiques, plus ou moins permanentes, du Saguenay-Lac- St-Jean, on verra successivement, dans cette deuxième section, le complexe relief - climat -eaux - sols, les ressources énergétiques et les ressources minérales, laissant pour le deuxième chapitre l'étude des éléments variables, en particulier le facteur humain.

\subsection{Le complexe relief - climat - eaux - sols}

Comme il a été dit plus haut, le Saguenay_Lac-St-Jean appartient à l'unité structurale du Bouclier Canadien. Il bénéficie d'un « relief calme » qui, selon R. Blanchard, caractérise toute la province du Québec. Par la dépression du Lac St-Jean, la région constitue cependant une unité naturelle nettement tranchée, dont la partie habitée est « une sorte d'oasis plantée au milieu de la rude nature laurentienne $» 32$.

Cette nature est en effet particulièrement sévère eu égard à son climat continental, aux hivers excessivement rigoureux et aux étés relativement chauds. Pour en donner une idée, on indiquera que Chicoutimi, dans les environs du Lac St-Jean, enregistre une moyenne de moins $16,2^{\circ} \mathrm{C}$ en hiver ; un peu plus au nord, Albanel atteint la moyenne de $-17,2^{\circ} \mathrm{C}$. En été, par contre, la moyenne de juillet à Chicoutimi dépasse $17^{\circ} \mathrm{C}$; il en est de même pour Albanel, dont la moyenne de juillet est de $17,1^{\circ} \mathrm{C}$. Il faut encore ajouter, à côté de ces températures extrêmes, la brièveté de la saison

31 J. Lebret, Dynamique concrète du développement, Éditions Ouvrières, Paris, 1963, p. 196.

32 R. Blanchard, op. cit., p. 59. 
utile pour les activités agricoles : 100 à 120 jours pour une année, soit de la deuxième quinzaine de mai à la mi-septembre. Ces conditions défavorables sont heureusement compensées par l'abondance des eaux et la fertilité des sols.

Le Saguenay_Lac-St-Jean est pourvu d'un réseau hydrographique riche et bien distribué 33. Le Lac St-Jean, à lui seul, constitue un vaste réservoir naturel de près de 410 milles carrés de superficie, soit environ deux fois celle du Lac Léman. Son émissaire, la rivière Saguenay, coule d'ouest en est et est navigable sur la plus grande partie de sa longueur, soit sur une distance de 68 milles. En outre, les rivières convergeant vers ces deux châteaux d'eau constituent une constellation remarquable. Pour n'en citer que les plus importantes, on retiendra la Chamouchouane, la Péribonka et le Shipshaw. On ne soulignera jamais assez l'importance du facteur hydrologique qui deviendra, le moment venu, le principal moteur de la région. Mais, comme pour tout processus de développement, c'est sur les sols que devaient se porter les premiers efforts de production.

33 Carte 2 page suivante. 
Carte 2

Le réseau hydrographique du Saguenay_Lac-St-Jean

$\underline{\text { Retour à la table des matières }}$

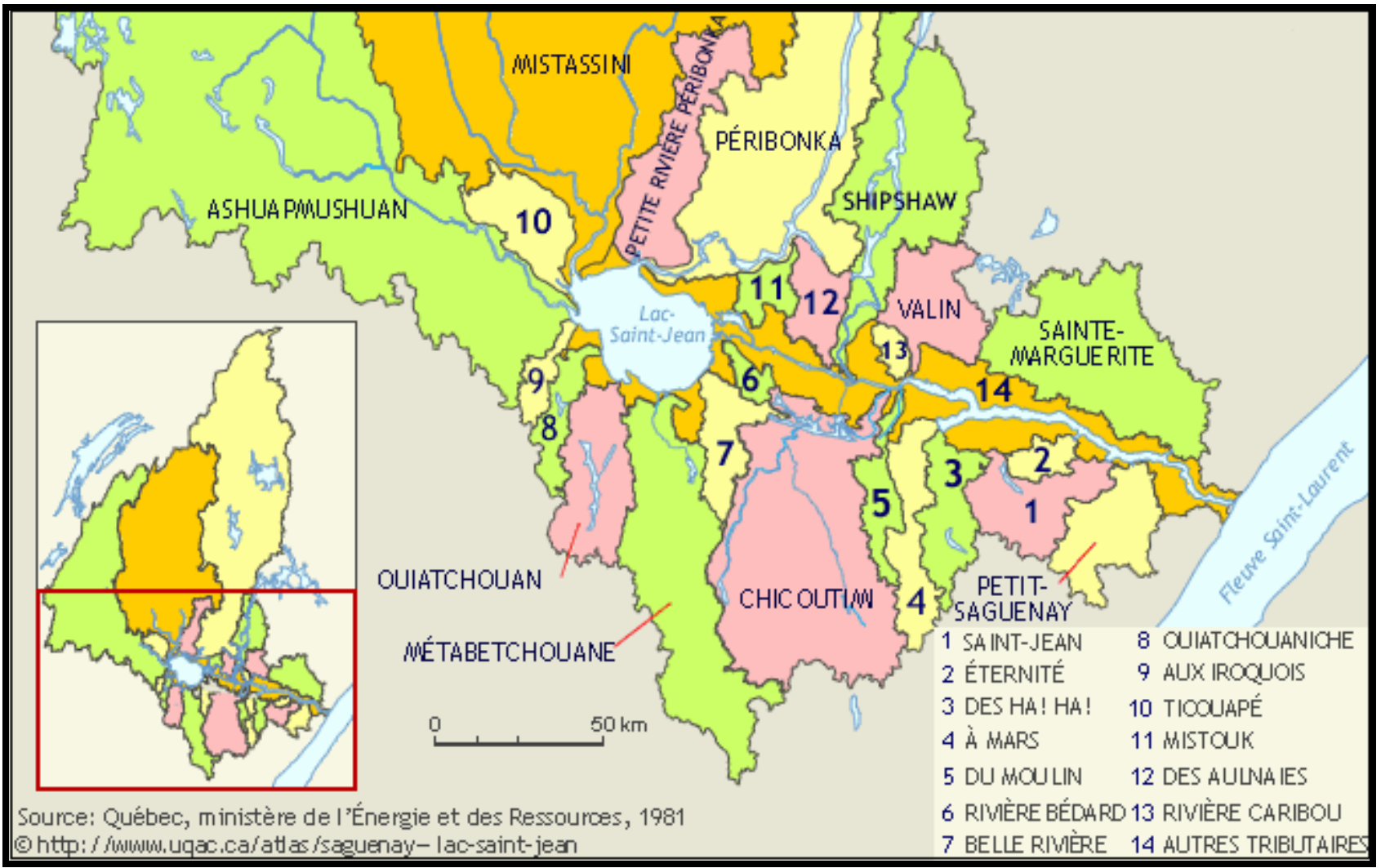


Or, ces sols, de l'avis de tous les observateurs, sont immenses et fertiles. Pour sa part, Raoul Blanchard a noté : « Aucune partie de la Province du Québec n'a été, touchant l'agriculture, l'objet d'aussi enthousiastes appréciations que la région du Saguenay-Lac-St-Jean. On l'appelle communément le Grenier de la Province » 34. Les caractéristiques pédologiques de la région, notamment de la partie du Lac St-Jean, ont été mises en évidence par des recherches plus récentes 35. Ce qui attire de prime abord l'attention, c'est la grande variété des sols, que les experts ont classés en quatre types principaux : « les sols lourds, les sols francs, les sols légers et les sols organiques ». On n'entrera pas ici dans les détails techniques de ces travaux, mais, on est en mesure de retenir que le caractère alluvial des terres basses, qui s'étendent sur le pourtour du Lac, et leur richesse en azotes les rendent très propres aux grandes cultures. Recouverts, durant le long hiver du pays d'un manteau de neige, arrosés de maintes précipitations (un mètre d'eau en moyenne par année), les sols du Saguenay_Lac-St-Jean sont peut-être les plus favorables qui soient dans l'Est de la Province.

Mais, « l'enclave agricole du Lac St-Jean et de la frange des Ha ! Ha ! n'est qu'un accident de la nature au sein d'un milieu géographique véritablement forestier » 36. La région se situe en effet au cœur de la grande zone forestière des Laurentides. Les bois les plus variés et les plus riches y abondent: tels «le merisier, le bouleau, le tremble, des érables, parmi les bois francs; les conifères y sont plus nombreux encore, tels le Pin blanc, le Pin rouge, le Pin gris, le sapin baumier et diverses épinettes » 37. D'après le Service de l'Aménagement Forestier du Québec, la superficie totale des bassins forestiers de la région est de 31322 milles carrés, dont 91,3 \% est considérée comme productive 38. Il y a là, à n'en pas douter, d'immenses possibilités pour une puissante industrie du bois et de ses dérivés.

34 R. Blanchard, op. cit., p. 44.

35 Aubé, Mailloux et Raymond, Pédologie de la région du Lac St-Jean, Québec, 1965, Ministère de l'Agriculture et de la Colonisation.

36 P.-Y. Pépin, Le Royaume du Saguenay en 1968, op. cit., p. 223.

37 R. Blanchard, L’Est du Canada français, op. cit., p.283.

38 P.-Y. Pépin, op. cit., p. 226. 


\subsection{Les ressources énergétiques}

$\underline{\text { Retour à la table des matières }}$

Après les richesses forestières, les ressources hydrauliques constituent la plus importante potentialité du Saguenay_Lac-St-Jean. C'est également la principale, sinon la seule, source connue d'énergie disponible dans la région. Cette richesse restait cependant quasi inexploitée. En effet, si l'on en croit R. Blanchard, «vers 1922, les forces hydrauliques aménagées dans le Saguenay-Lac-St-Jean représentaient à peu près 117000 chevaux-vapeur, dont 72000 à la disposition de la Maison Price » 39. Cette Maison, la plus importante de l'ensemble d'industries papetières qui constituaient alors l'essentiel de l'industrie régionale, possédait ainsi plus de la moitié de l'énergie disponible. La distribution de la capacité hydroélectrique saguenayenne se présentait alors comme suit :

Tableau 2

Capacité hydroélectrique au Saguenay, 1922

\begin{tabular}{|l|c|}
\hline Rivières Chicoutimi et aux Sables & 74650 c.v. \\
\hline Rivière Shipshaw & 28400 c.v. \\
\hline Val-Jalbert & 7300 c.v. \\
\hline Métabetchouan & 1400 c.v. \\
\hline Petite Péribonka & 1500 c.v. \\
\hline Ha Ha et Rivière à Mars & 3400 c.v. \\
\hline Total & $\mathbf{1 1 6} \mathbf{6 5 0}$ c.v. \\
\hline
\end{tabular}

Source : Tiré du tableau des Forces Hydrauliques de la Province, 1928, cité par Raoul Blanchard: idem, pp. 108-109.

Si on a gardé à la mémoire ce qui a été dit au paragraphe précédent, l'abondance du réseau hydrographique du Saguenay-Lac-St-Jean, le tableau des forces hydroélectriques

39 R. Blanchard, idem, p. 98. 
présenté plus haut témoigne d'une faiblesse à première vue paradoxale. À la vérité, la sousexploitation des potentialités existantes en ce domaine trouve une première explication dans l'état de sous-industrialisation qui caractérisait alors la région. Cette observation demande cependant certaines précisions. En effet, pendant longtemps, l'insuffisance de l'énergie électrique et, surtout, le caractère irrégulier de l'approvisionnement avaient constitué un frein non négligeable pour le développement de l'industrie régionale embryonnaire. La faiblesse de l'industrialisation de la région n'explique donc qu'imparfaitement les carences de la production énergétique. C'est plutôt l'insuffisance des capitaux disponibles, alliée à un manque de tradition industrielle, et par conséquent d'entrepreneurs dynamiques, qui explique le mieux le retard apporté à la mise en valeur des richesses hydrauliques du Saguenay-Lac-St-Jean. L'afflux postérieur des capitaux américains dans le circuit économique de la région allait modifier la situation du tout au tout. On aura ainsi à revenir sur le problème de l'énergie, dont le rôle a été déterminant dans l'évolution saguenayenne.

\subsection{Les ressources minérales}

$\underline{\text { Retour à la table des matières }}$

Du point de vue des richesses du sous-sol, le Saguenay_Lac-St-Jean n'apparaît pas particulièrement bien pourvu. Certes, des études géologiques 40 ont montré qu'il abonde en granit, pierre très recherchée pour la décoration. Les principaux gisements connus s'étendent aux abords du Lac St-Jean, dans les environs de Roberval, du côté de Chicoutimi et de la Baie des Ha! Ha! La région possède en outre d'immenses carrières de gravier, très répandu dans tout le Saguenay-Lac-St-Jean et qui constitue un matériau de premier choix pour la construction de routes et autres gros oeuvres. Ces ressources étaient connues, et sur une base artisanale, faisaient l'objet d'une certaine exploitation, bien avant l'arrivée de

40 J.A. Dresser et T.C. Denis, Géologie du Québec, Vol. III, Géologie économique, pp. 597-599. 
l'Alcan dans la région. Il n'y avait pas là toutefois les matières premières susceptibles de donner naissance à une industrie lourde et puissante.

Des recherches plus récentes 41 ont cependant révélé l'existence d'importantes ressources minières, notamment dans la zone de Chibougamau qui, comme on l'a déjà souligné, a été rattachée au Saguenay-Lac-St-Jean en 1951. Chibougamau s'étend sur la partie la plus au nord de la région géologique du Témiscamingue, la zone la plus minéralisée de l'Ouest du Québec. Ses gisements de cuivre, avec traces d'or et d'argent, ont donné lieu à une intense exploitation. En 1967, l'ensemble des réserves connues était évalué à une vingtaine de millions de tonnes, dont la teneur en cuivre serait en moyenne de 2,50\%. La région posséderait en outre des minerais d'amiante, de titane, de fer et d'uranium. Mais, ici, on est beaucoup plus sur le terrain des hypothèses que de la réalité. Quoiqu'il en soit, dans les années 1920, ces ressources étaient pour la plupart inconnues et ne donnaient par conséquent lieu à aucune activité économique.

Au total, on peut caractériser du signe « plus » le bilan de ce rapide inventaire des potentialités physiques du Saguenay-Lac-St-Jean : un territoire immense, doté de sols variés et propres à l'agriculture, de ressources forestières étendues favorables à l'industrie du bois et du papier, enfin d'un atout majeur, l'énergie hydroélectrique. Nombre de pays aujourd'hui industrialisés ne disposent pas de tous ces avantages. Mais, au-delà des richesses naturelles, il existe un facteur qui conditionne, plus que tout autre, la croissance économique : ce sont les hommes.

41 Ministère des Mines, Empire minier - Aperçu de la géologie et des ressources minérales de la Province de Québec, Québec, 1958. 
Première partie : Le Saguenay_Lac-St-Jean avant l'implantation de l'Alcan

\section{Chapitre II}

\section{Le Saguenay-Lac-St-Jean : les éléments humains}

$\underline{\text { Retour à la table des matières }}$

Aussi bien qu'il n'y a pas d'activité économique sans hommes, aussi bien une analyse de la croissance économique se doit d'être précédée d'une étude du facteur humain. Le Professeur Perroux apporte sa caution en ce sens, quand, soulignant l'importance du «dynamisme du psychisme humain » dans le domaine économique, il écrit : «Dès que l'économie est considérée comme une activité d'hommes concrets et non comme un ajustement mécanique des quantités, elle s'offre à nous travaillée par les dynamismes de la vitalité et par les dynamismes de la personnalité » 42. On peut laisser la passionnante tâche de l'analyse des éléments psychiques aux experts de la question. Il importe cependant pour ce travail de faire un diagnostic du problème humain, en particulier de la main-d'œuvre de la région, avant de tenter de dégager dans une brève conclusion les traits caractéristiques de l'économie du Saguenay_Lac-St-Jean avant l'implantation de l'Alcan. Ce chapitre comprendra deux grandes sections: dans la première, on étudiera les problèmes démographiques généraux de la région et dans la seconde, on se penchera sur les problèmes particuliers de la main-d’œuvre.

42 F. Perroux, L’économie du XXe siècle, op. cit., p. 469. 


\section{Section 1 : La démographie régionale}

\subsection{Les chiffres de population}

\section{$\underline{\text { Retour à la table des matières }}$}

Ce qui frappe au départ l'observateur, c'est d'un côté la discontinuité du peuplement régional et de l'autre l'extrême jeunesse de la population. C'est que le Saguenay_Lac-St-Jean, pour reprendre l'expression des géographes, est une zone de colonisation récente. Raoul Blanchard écrit à ce propos : « Les premiers Canadiens à risquer un établissement le long du Saguenay, des paysans de la Malbaie, se présentèrent comme bûcherons et commencèrent en effet en 1838 à couper du pin blanc au fond de la Baie Ha ! Ha ! » 43. Dans la même veine, l'auteur, citant un Rapport sur les Missions des Diocèses du Québec, affirme que, vers 1845, il n'y avait que 3000 hommes dans toute la région du Saguenay_Lac-St-Jean. En dépit d'une évolution relativement rapide, la région témoigne encore de cette faiblesse démographique, comme il ressort du tableau suivant.

Tableau 3

SLSJ : population 1851-1921

\begin{tabular}{|l|c|c|c|c|}
\hline & $\mathbf{1 8 5 1}$ & $\mathbf{1 8 7 1}$ & $\mathbf{1 8 9 1}$ & $\mathbf{1 9 2 1}$ \\
\hline Chicoutimi & 5199 & 11812 & 14244 & 37578 \\
\hline Lac St-Jean & 150 & 5681 & 14048 & 35539 \\
\hline Total région & $\mathbf{5 3 4 9}$ & $\mathbf{1 7 4 9 3}$ & $\mathbf{2 8 2 9 2}$ & $\mathbf{7 3 \mathbf { 1 1 7 }}$ \\
\hline & & & & \\
\hline & $\mathbf{1 9 3 1}$ & $\mathbf{1 9 5 1}$ & $\mathbf{1 9 6 1}$ & $\mathbf{1 9 6 6}$ \\
\hline Chicoutimi & 55924 & 115904 & 157196 & 167773 \\
\hline Lac St-Jean & 50253 & 82006 & 105230 & 105909 \\
\hline Total région & $\mathbf{1 0 5 9 7 7}$ & $\mathbf{1 9 7 9 1 0}$ & $\mathbf{2 6 2} \mathbf{4 2 6}$ & $\mathbf{2 6 7 6 8 2}$ \\
\hline
\end{tabular}

Source : BFS, Canada.

43 R. Blanchard, Le Canada français, op. cit., p. 96. 
Figure 1

Évolution du volume de population du Saguenay-Lac-St-Jean 1851-1966

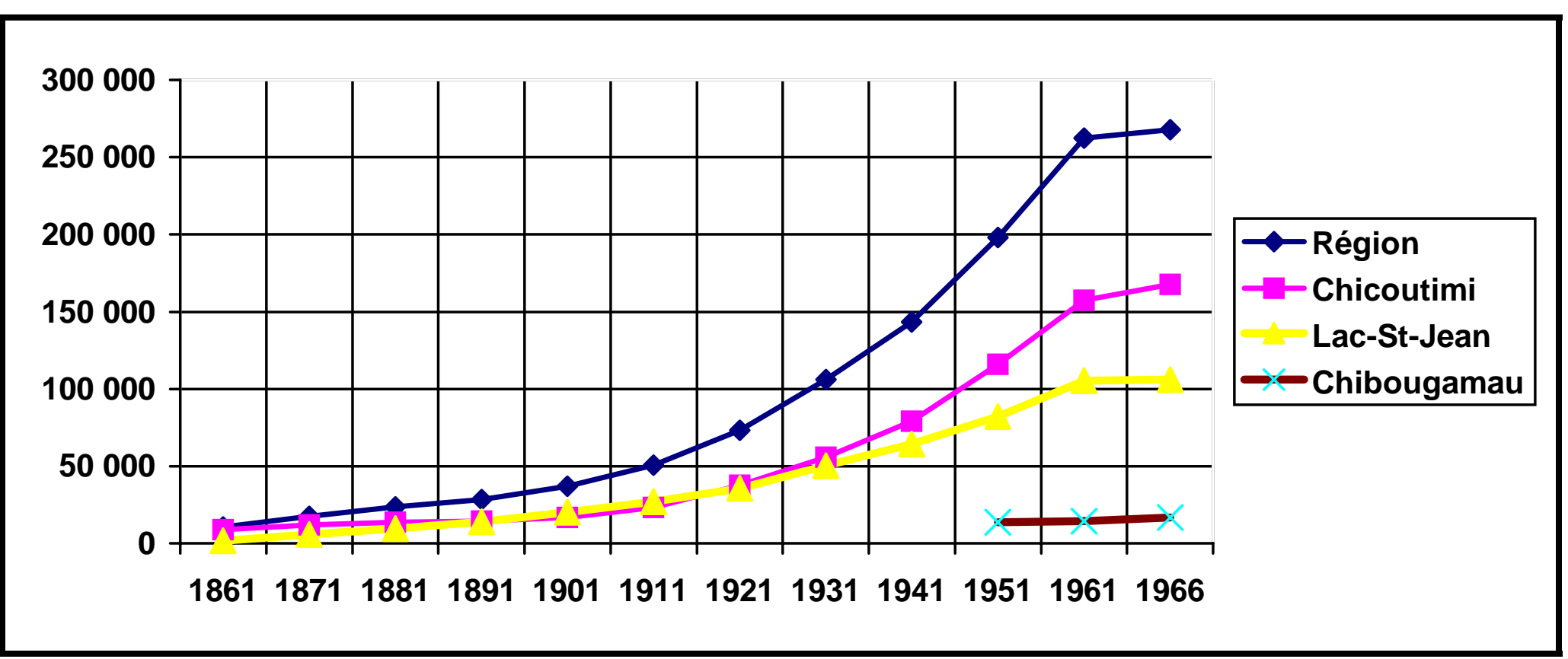

Source : BFS, Canada, dans P.-Y. Pépin, op. cit., p. 126. 
Si on a encore à l'esprit les données du chapitre précédent concernant l'étendue du territoire régional, l'examen même rapide du tableau 3 révèle l'extrême faiblesse quantitative de la population du Saguenay_Lac-St-Jean. Certes, la population a témoigné d'un dynamisme certain dans son accroissement. Lente, jusqu'au début du XXe siècle, l'évolution a pris une allure rapide à partir des années 1920. Sur la base des données fournies au tableau précité, il a été construit la figure 1 qui traduit le rythme relativement vigoureux de l'accroissement démographique régional.

Il importe de faire ressortir certains éléments qui paraissent très instructifs relativement à cette évolution. C'est d'abord un renversement de la tendance, à l'avantage du Lac St-Jean, d'un peuplement qui avait commencé dans le Saguenay. Du reste, cette situation ne sera maintenue que fort peu de temps, soit durant la période allant de 1891-92 à 1919-20. Mais, à partir de ces deux dernières années qui coïncident avec les débuts de l'industrialisation de la région, le comté de Chicoutimi gardera l'avance sur le comté plus agricole du Lac St-Jean. Quoiqu'il en soit, en 1921, peu de temps donc avant que l'Alcan n'arrive sur la scène économique régionale, la population globale du Saguenay_Lac-St-Jean s'élevait à 73117 habitants, partagés entre le comté de Chicoutimi (37 578) et le Lac St-Jean (35 539). Cette population était relativement homogène, puisque les éléments d'expression française en représentaient près de 95\%. Elle était également jeune. Ce dernier point sera mis encore plus en relief dans l'étude de la structure de la population en rapport avec la main-d’œuvre.

\subsection{Structure de la population}

Le régime démographique du Saguenay_Lac-St-Jean suit de près celui de la province du Québec. Or, ce qui caractérise la population québécoise, c'est sa grande jeunesse et, jusqu'à récemment, un dynamisme exceptionnel de la natalité. Cela s'explique, en partie tout au moins, par le fait que cette population s'est constituée, au départ, par des afflux successifs d'immigrants qui sont généralement jeunes et pleins de vitalité. La figure 2 donne une assez juste idée de la composition de la population régionale. Il s'agit de deux pyramides d'âges superposées de la Province du Québec pour 1881 et 1951. 
Figure 2

Pyramide d’âge de la population de la province de Québec, 1881 et 1951

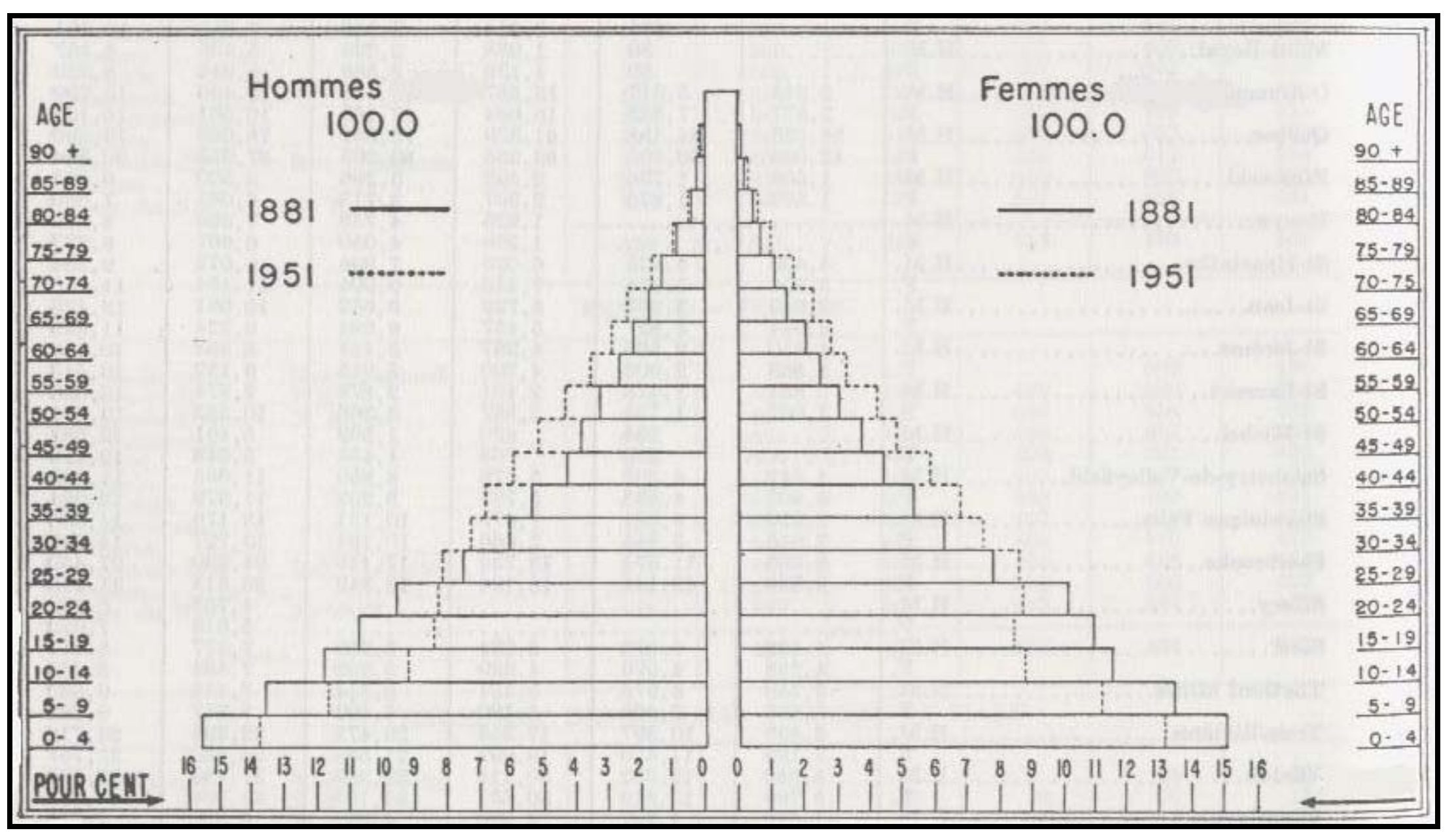


Pour les deux années considérées, il est remarquable de voir combien sont larges les bases des pyramides des âges de la province du Québec. Cependant, entre 1881 et 1951, sont apparus des signes d'une certaine maturité, comme la diminution notable des classes d'âges variant entre 1 et 25 ans. Ce phénomène n'est pas spécifique au Québec. On peut certes en faire la constatation dans la plupart des pays où l'élévation du niveau de vie s'accompagne généralement d'une baisse de la natalité de la population. On peut admettre que la population du Saguenay-Lac-St-Jean, à quelques différences près, présentait les mêmes caractéristiques. En fait preuve, du reste, le tableau suivant qui compare les régimes démographiques du Québec et de la région pour des années plus récentes.

Tableau 4

Structure par âges de la population au Québec et au Saguenay—Lac-St-Jean

\begin{tabular}{|c|c|c|c|c|c|c|c|c|}
\hline $\begin{array}{c}\text { Groupes } \\
\text { d'âge }\end{array}$ & $\begin{array}{c}\text { Région } \\
1961 \\
\text { H }\end{array}$ & $\begin{array}{c}\text { Région } \\
1961 \\
\text { F }\end{array}$ & $\begin{array}{c}\text { Québec } \\
1961 \\
\text { H }\end{array}$ & $\begin{array}{c}\text { Québec } \\
1961 \\
\text { F }\end{array}$ & $\begin{array}{c}\text { Région } \\
1966 \\
\text { H }\end{array}$ & $\begin{array}{c}\text { Région } \\
1966 \\
\text { F }\end{array}$ & $\begin{array}{c}\text { Québec } \\
1966 \\
\text { H }\end{array}$ & $\begin{array}{c}\text { Québec } \\
1966 \\
\text { F }\end{array}$ \\
\hline $0-4$ & 8,05 & 7,75 & 6,50 & 6,20 & 3,75 & 3,55 & 5,35 & 5,65 \\
\hline 5-9 & 7,45 & 7,15 & 6,00 & 5,80 & 7,75 & 7,45 & 5,80 & 6,05 \\
\hline $10-14$ & 6,60 & 6,25 & 5,50 & 5,20 & 7,20 & 6,80 & 5,30 & 5,55 \\
\hline $15-19$ & 5,45 & 5,25 & 4,45 & 4,40 & 6,40 & 5,90 & 4,85 & 4,90 \\
\hline $20-24$ & 3,90 & 3,85 & 3,40 & 3,60 & 4,95 & 4,65 & 4,15 & 4,00 \\
\hline $25-29$ & 3,50 & 3,40 & 3,50 & 3,40 & 3,60 & 3,30 & 3,35 & 3,25 \\
\hline $30-34$ & 3,25 & 3,25 & 3,55 & 3,60 & 3,20 & 3,10 & 3,20 & 3,20 \\
\hline 35-39 & 3,00 & 2,85 & 3,35 & 3,50 & 3,10 & 3,05 & 3,30 & 3,20 \\
\hline $40-44$ & 2,45 & 2,25 & 2,90 & 2,90 & 2,85 & 2,70 & 3,10 & 3,05 \\
\hline $45-49$ & 2,00 & 1,80 & 2,65 & 2,60 & 2,20 & 2,20 & 2,60 & 2,55 \\
\hline $50-54$ & 1,60 & 1,45 & 2,30 & 2,20 & 1,90 & 1,70 & 2,35 & 2,30 \\
\hline $55-59$ & 1,30 & 1,15 & 1,70 & 1,80 & 1,45 & 1,35 & 1,90 & 1,90 \\
\hline $60-64$ & 1,00 & 0,80 & 1,30 & 1,40 & 1,10 & 1,00 & 1,60 & 1,45 \\
\hline 65-69 & 0,70 & 0,55 & 1,00 & 1,10 & 0,85 & 0,70 & 1,25 & 1,10 \\
\hline $70-74$ & 0,50 & 0.45 & 0,70 & 0,80 & 0,55 & 0,40 & 0,90 & 0,80 \\
\hline \multirow[t]{3}{*}{75 et +} & 0,55 & 0,50 & 0,80 & 0,90 & 0,65 & 0,55 & 1,15 & 0,90 \\
\hline & 51,30 & 48,70 & 49,60 & 50,40 & 51,60 & 48,40 & 50,15 & 49,85 \\
\hline & $100 \%$ & $100 \%$ & $100 \%$ & $100 \%$ & $100 \%$ & $100 \%$ & $100 \%$ & $100 \%$ \\
\hline
\end{tabular}

Source ; OPDQ, Variables économiques du SLSTJ, 1969, page 6. 


\section{Section 2 : La main-d'œuvre régionale}

\subsection{Le problème structurel et quantitatif}

$\underline{\text { Retour à la table des matières }}$

Des développements qui précèdent, il est possible de tirer au moins une conclusion, à savoir que la faiblesse numérique et la jeunesse de la population de la région - plus de 50\% âgés de moins de vingt ans en 1961 - laissent une population active relativement peu considérable. On pressent déjà les conséquences que cette situation peut entraîner pour la main-d’œuvre et le niveau des revenus régionaux.

Jusqu'au début du XXe siècle, l'agriculture et l'exploitation brute des ressources naturelles ont constitué l'essentiel de l'activité économique du Saguenay-Lac-St-Jean. Cette situation, bien connue dans les pays peu développés, n'était pas, à la vérité, spécifique à cette région. Elle caractérisait certes l'économie de tout le Québec et même, dans une mesure moindre cependant, celle du Canada. Jusqu'en 1941, précise R. Blanchard, la province du Québec comptait 839000 hommes occupés aux tâches agricoles, ce qui représentait plus de 25\% de la population totale 44 . De son côté, R. Parenteau, soulignant l'immaturité de l'économie canadienne, écrit : « En dépit du très haut niveau de vie dont jouissent les habitants, en dépit du fait que certaines industries ultramodernes ne le cèdent en rien à celles des pays les plus avancés, l'économie canadienne manque singulièrement de maturité » 45. Cette immaturité transparaît au travers de la structure de la main-d'œuvre, marquée par la forte proportion des travailleurs du secteur primaire et la faiblesse relative de la maind'œuvre proprement industrielle, comme on peut s'en rendre compte dans le tableau qui suit.

44 R. Blanchard, Le Canada français, op. cit., p. 137.

45 R. Parenteau, op. cit., pp. 172-173. 
Tableau 5

\section{Répartition de la population canadienne selon les occupations}

\begin{tabular}{|l|c|c|c|}
\hline Secteurs & Année & Année & Année \\
\hline & $\mathbf{1 8 9 1}$ & $\mathbf{1 9 2 1}$ & $\mathbf{1 9 5 1}$ \\
\hline Primaire & $56,3 \%$ & $46,1 \%$ & $26,0 \%$ \\
\hline Secondaire & $20,1 \%$ & $17,9 \%$ & $24,9 \%$ \\
\hline Tertiaire & $23,4 \%$ & $35,7 \%$ & $47,8 \%$ \\
\hline
\end{tabular}

Source : Roland Parenteau, idem, page 173.

En ce qui concerne le Saguenay_Lac-St-Jean et pour la période sous référence, on ne dispose pas de données aussi précises. Cependant, il est possible de se faire une idée valable de la maind'œuvre régionale en partant d'autres considérations, notamment de la répartition de la population selon le critère d'urbanisation. Ce critère ne définit pas à coup sur le type d'occupation. Mais, il y a une coïncidence significative entre les tâches dites primaires et le caractère rural, entre les activités secondaires-tertiaires et le caractère urbain.

Or, en 1921, sur un total de 73117 habitants, répartis pour 37578 à Chicoutimi et 35539 au Lac St-Jean, le pourcentage d'urbanisés était relativement faible. Les deux comtés du Lac St-Jean accusaient 25\% d'urbains contre 75\% de ruraux alors que le comté de Chicoutimi, plus urbanisé, comptait 62\% de citadins. Ces pourcentages sont malgré tout relativement trompeurs. En effet, seule l'agglomération de Chicoutimi, avec ses 10000 habitants, pouvait être réellement considérée comme une ville, toutes les autres localités n'étant que de grosses bourgades. Une exception cependant pouvait être faite pour Kénogami et Jonquière, deux petites villes voisines, facilement jumelables.

Partant de ces considérations, il est aisé de conclure que le gros de la main-d'œuvre régionale, pour cette période, devait s'occuper à des tâches primaires. Cette observation se confirme du reste, à la lumière de la structure de la main-d'œuvre de la région, telle qu'elle apparaît à travers les statistiques disponibles pour l'année 1941. 
Tableau 6

Région : main-d'œuvre selon l'occupation, 1941

\begin{tabular}{|l|c|c|c|}
\hline Secteurs & Chicoutimi & Lac St-Jean & Main-d'œuvre \\
\hline Agriculture & 4451 & 8250 & 12701 \\
\hline Forêts, mines, etc. & 1411 & 1832 & 3243 \\
\hline Journaliers & 4274 & 1334 & 5608 \\
\hline Construction & 2085 & 789 & 2872 \\
\hline Manufactures & 4572 & 1688 & 6260 \\
\hline Transports & 1391 & 751 & 2142 \\
\hline Commerce, finance & 1622 & 952 & 2574 \\
\hline Services & 3895 & 2383 & 6278 \\
\hline Commis de bureau & 1053 & 376 & 1429 \\
\hline Total & $\mathbf{2 4 8 9 1}$ & $\mathbf{1 8 4 2 2}$ & $\mathbf{4 3 3 1 3}$ \\
\hline
\end{tabular}

Source : BFS, Canada, cité par P.Y. Pépin : op. cit., p. 117.

En 1941, la main-d'œuvre totale de la région s'élevait donc à 43313 unités, volume relativement faible quand on le rapporte à la population globale d'alors, soit 143187 habitants. On sait désormais que cette faiblesse tenait pour une bonne part à la jeunesse de cette population. Par ailleurs, les remarques faites plus haut quant à la structure de l'emploi dans la région en 1921 semblent valables, puisqu'elles sont encore justifiées vingt ans plus tard. En effet, si on classe dans le secteur primaire les activités agricoles, forestières et minières, 15944 personnes y trouvaient leur emploi en 1941, soit environ 37 \% du volume total de main-d'œuvre. Mais, à la vérité, ce secteur était d'un poids relatif plus important encore, si on y ajoute les «journaliers », dont les tâches se situaient «à mi-chemin entre la mise en valeur des ressources naturelles et l'industrie proprement dite» 46. Le seul secteur primaire représenterait ainsi $50 \%$ environ du volume régional de main-d'œuvre. Le secondaire, quant à lui, compterait les employés des manufactures et de la construction, soit un total de 9132 hommes ou 21\% de l'emploi régional. Ainsi, il ne resterait au secteur tertiaire que 12423 travailleurs, soit 29\% du volume global de main-d'œuvre.

46 B. Brouillette, « L’habitat et la population au Saguenay », dans L'Actualité économique, no 4, janvier 1947, p. 664. 
Cette structure de l'emploi régional révèle un fait important, à savoir que, si le Saguenay—LacSt-Jean n'était pas une région sous-développée au sens premier du terme, c'est-à-dire pauvre, il n'était pas non plus une région de grande industrialisation. Certes, on peut relever, pour 1941, que 45\% de la main-d’œuvre régionale gagnaient entre 1000 et 2000 dollars ; 50\% touchaient un peu moins de 1000 dollars et les 5\% restants, plus de 2000 dollars. Il s'agit, bien entendu, de salaires annuels. Sur l'ensemble de la population active, on ne comptait qu'un millier de chômeurs, soit le pourcentage relativement peu élevé de $4 \%$. Somme toute, on peut bien dire que sous certains aspects la région se rapprochait davantage des pays industriels que des pays sousdéveloppés 47.

\subsection{Le problème qualitatif de la main-d'œuvre}

\section{$\underline{\text { Retour à la table des matières }}$}

Cette partie de l'étude se révèle particulièrement délicate, vu l'inexistence de données précises sur les problèmes régionaux de l'enseignement en général et de la formation professionnelle en particulier, du moins pour la période sous référence. Cette difficulté a été signalée par P.-Y. Pépin qui, traitant de l'organisation de l'enseignement dans $L e$ royaume du Saguenay en 1968, écrit : « l'approche est tout particulièrement ingrate sur le plan des effectifs scolaires, données fuyantes bien que le système d'enseignement luimême... bénéficie de profondes transformations » 48. C'est déjà là un indice de la faiblesse du système qui avait prévalu jusque-là dans la Province. On peut rappeler, à cet effet, que la loi de l'instruction obligatoire ne date que de 1943. Il faut se résigner à restreindre l'analyse aux possibilités de la documentation. Or, ce qui semble caractériser l'enseignement dans la province du Québec, c'est l'influence traditionnellement prépondérante de l'Église catholique. Cette institution a eu la haute main sur tout le système

\footnotetext{
47 B. Brouillette, «L'habitat et la population au Saguenay », dans L'Actualité économique, no 4, janvier 1947, p. 664.

48 P.-Y. Pépin, op. cit., p. 137.
} 
d'éducation, depuis la fondation du Canada français jusqu'encore récemment. R. Blanchard a résumé la situation ainsi :

L'Église a complètement gardé la maîtrise de l'enseignement. Elle contrôle de très près les écoles primaires, ainsi que les écoles normales d'où sortent les maîtres, par l'autorité du Conseil Provincial de l'Instruction Publique, dont font partie, de droit, tous les évêques assistés d'un nombre égal de laïcs choisis par l'épiscopat. Elle dirige les petits séminaires et collèges où se dispense l'enseignement secondaire. Les trois universités françaises de la Province sont catholiques, leurs recteurs sont des prélats, leurs chanceliers sont l'archevêque du diocèse, beaucoup de leurs professeurs sont des religieux49.

On peut aisément deviner que ce système d'enseignement n'était pas naturellement tourné vers la formation scientifique, technique et professionnelle. Peut-être, compte tenu de l'échelle des préférences collectives des populations de l'époque, il répondait à d'autres objectifs tout aussi importants. Mais, il explique en partie l'arrivée plutôt tardive du Québec dans le peloton des pays industriels. Il n'est pas non plus étonnant, comme le souligne encore Blanchard, que « les vrais leviers de commande soient aux mains d'étrangers confondus sous le vocable Anglais, tandis que les Français sont relégués dans les professions libérales, le petit commerce, l'agriculture, les emplois mineurs de l'industrie... L'enseignement pratiqué dans la Province en est largement responsable » 50.

Ainsi, on peut admettre qu'avant l'implantation de l'Alcan la main-d'œuvre du SaguenayLac-St-Jean n'avait pas un degré très élevé de qualification professionnelle. L'enseignement technique y était très faible; l'université était réservée à une mince élite qui se tournait plus volontiers vers les études littéraires que vers les carrières scientifiques, plus facilement vers les professions libérales que vers les tâches industrielles. Ce n'est donc pas sans raison que certains auteurs vont jusqu'à considérer l'industrialisation du Québec, dans le premier quart du XXe siècle, comme « la simple manifestation régionale de l'ensemble de l'évolution économique du continent nord-américain » 51. De fait, les structures d'accueil et les mentalités étaient peu favorables au

49 R. Blanchard, Le Canada français, op. cit., p. 295.

50 R. Blanchard, idem, p. 297.

51 A.Faucher, M. Lamontagne, «History of Industrial Development », dans M. Rioux, A. Martin, Edition French Canadian Society, Vol. I, The Carlton Library, no 18, 1966, p. 257. 
phénomène nouveau de l'industrialisation qui , en dépit de la faible participation initiale des éléments francophones largement majoritaires, allait se développer dans cette Province aux potentialités immenses. Cette évolution ne manquera pas d'entraîner de profondes transformations sur les esprits et la façon de vivre des populations.

Au terme de cette première partie de l'étude, il est bon de rappeler l'objectif fixé au départ, à savoir, dégager les caractéristiques fondamentales du Saguenay-Lac-St-Jean avant l'implantation de l'Alcan dans cette région. En résumé, au travers des développements qui précèdent, on a découvert une région naturellement bien pourvue, mais relativement faible du côté de son potentiel humain. On a décelé une certaine fragilité des structures d'une économie peu diversifiée, reposant essentiellement sur l'agriculture et quelques petites industries basées sur l'exploitation des ressources forestières. Certaines parties de ces analyses auront paru, par moments, longues et laborieuses. Mais, on a essayé d'expliquer chaque fois les démarches y présidant, dont l'ultime justification peut être trouvée dans cette observation si à propos de M. Penouil : «Le phénomène de polarisation parait jouer un rôle essentiel dans le processus de développement, mais l'amplitude de ces effets et leurs modalités dépendent, dans une large mesure, des réactions du milieu » 52. Maintenant que le milieu a été présenté, il importe d'aborder les composantes de la machine financière et industrielle de l'Alcan. Ce sera l'objet essentiel de la deuxième partie de cette étude.

52 M. Penouil, Pôles de développement en régions sous-développées et en pays sous-développés, Colloque du 18 au 23 mai 1968, Genève, p. 45. 


\section{DEUXIÈME PARTIE}

\section{L’Alcan et l'évolution de l'économie du Saguenay -Lac-St-Jean}

$\underline{\text { Retour à la table des matières }}$

L'observation des rapports entre une unité industrielle de production et un ensemble économique plus vaste, régional ou national, est une tâche délicate. Cela provient de ce que les deux ordres de fait obéissent à des dynamiques spécifiques et à des motivations distinctes. Ainsi en est-il de l'Alcan et de l'économie du Saguenay_Lac- St-Jean, dont l'évolution diffère aussi bien dans le temps que dans l'espace. D'un côté, la vie économique régionale est antérieure à l'implantation de la société Alcan ; de l'autre, le cadre spatial des activités économiques de la Compagnie déborde largement celui de la région. Cette double différence, pour ne souligner que celle-là, suggère que la dépendance réciproque de ces deux réalités que sont l'Alcan et l'économie saguenayenne ne saurait être analysée qu'en termes très relatifs. Plus explicitement, cette étude n'envisagera que la période se situant entre 1925 (année de l'implantation de l'Alcan dans la région) et les années les plus 
récentes, en ce qui concerne les phénomènes économiques régionaux liés directement ou indirectement aux activités de cette Société.

Cette deuxième partie comprendra trois chapitres :

- l'implantation de l'Alcan dans la région ;

- la nature fondamentale de cette Société ;

- le repérage des indicateurs de la croissance économique régionale. 
Deuxième partie :

L’Alcan et l'évolution de l’économie du S.-L.-S-.J.

Chapitre III

\section{L’implantation de l'Alcan dans le Saguenay_Lac-St-Jean:}

$\underline{\text { Retour à la table des matières }}$

Selon J. Mac Neil, « la localisation de la firme résulte de la minimisation des coûts totaux pour une production donnée et de la maximisation des ventes, compte tenu d'un niveau minimum de profits » 53. Ce type de calcul traduit des préoccupations d'ordre microéconomique. Il exclut, en apparence tout au moins, les économies externes. L'entreprise, ne sachant pas en général les mesurer correctement, ne les intègre pas dans sa comptabilité. Mais, elle n'en recherche pas moins les avantages, lorsqu'elle est en quête d'un milieu d'accueil. Entre autres, la nouvelle entreprise essaie de s'implanter près de son marché potentiel ou à proximité de ses fournisseurs d'inputs, ce qui, souligne Jean le Pas, « constitue des économies externes, parce que le coût qu'elle supporterait en s'installant ailleurs serait grevé de frais de transport supplémentaires » 54.

Cela explique la coexistence, dans presque tous les pays, de zones sur-industrialisées et de régions économiquement déprimées. En ce domaine, les tendances naturelles des entreprises contrecarrent

53 J.M. Neil, cité par Maillat et Mathey, «Industrialisation et développement régional », Revue économique et sociale, juin 1970, p. 115.

54 J. Le Pas, Cahiers de l'ISEA, no 6, juin 1968, p. 1255. 
les meilleurs programmes établis par les pouvoirs publics, en vue de la décentralisation ou d'une moindre disparité entre les régions ou les composantes de la nation.

L'objectif de la rentabilité positive, c'est-à-dire la maximisation du profit de l'entreprise isolée de tout le contexte économique, est cependant la règle d'or de tout homme d'affaires. En termes de localisation, elle implique donc la proximité des sources de matières premières, d'énergie, du marché du travail et des produits. On doit souligner toutefois que la baisse des coûts de transports a atténué le caractère contraignant de ces conditions. La marge de manœuvre de l'entreprise contemporaine est certes plus importante que celle des fabriques du XIXe siècle. On n'est plus à l'époque où le cycle de production dépendait presque complètement des conditions géographiques. L'observation de Weber garde encore son sens, à savoir que « la répartition d'une branche d'activité dépend de la localisation des ressources, de la main-d’œuvre et du marché »55.

La présence ou l'absence de ces facteurs s'avère ainsi déterminante à l'entreprise pour le choix d'un site. Dépendant du type d'industrie, on accordera la priorité à l'un ou l'autre d'entre eux. Qu'en a-t-il été de l'Alcan, Société de production d'aluminium, dans le choix du Saguenay-Lac-St-Jean, comme site de ses activités ? La première démarche devra consister à dégager les traits caractéristiques de ce type d'industrie, les éléments techniques de la production d'aluminium.

55 A. Weber, Über den Standort der Industrien, cité par P. Claval, op. cit., p. 413. 


\section{Section 1. La métallurgie de l’aluminium}

$\underline{\text { Retour à la table des matières }}$

La fabrication de l'aluminium se fait principalement à partir de la bauxite, le plus riche en teneur des minerais d'oxyde d'aluminium très répandus dans la croûte terrestre. Elle tient son nom des Baux, village de Provence, en France, où les premiers gisements ont été découverts. La métallurgie de l'aluminium comporte deux phases essentielles : la première consiste, au moyen d'un procédé chimique devenu classique : le procédé Bayer, à extraire l'alumine de la bauxite et la seconde, à réduire l'alumine obtenue en aluminium par un procédé électrolytique.

\subsection{Le procédé Bayer}

On commence par broyer en poudre fine la bauxite qui est fondamentalement un composé d'oxyde d'aluminium hydraté et d'autres oxydes métalliques, de fer ou de silicium. Cette poudre est ensuite mise en contact dans une autoclave avec une solution de soude concentrée et chaude. L'oxyde d'aluminium se dissout dans la soude pour former une solution d'aluminate de sodium, tandis que les composés secondaires restent à l'état solide. Filtrée sous pression, la solution d'aluminate se sépare de ses impuretés, puis est décomposée en présence de cristaux d'alumine. Le produit ainsi obtenu est un précipité de trihydrate d'alumine, dont l'eau est chassée par calcination dans les fours rotatifs portés à environ mille degrés centigrades. Le résidu de cette première opération est l'oxyde d'aluminium anhydre ou plus simplement l'alumine calcinée.

\subsection{Le procédé électrolytique}

Cette deuxième phase de la métallurgie de l'aluminium est de loin la plus importante. Elle fait appel à d'énormes quantités d'énergie électrique : environ 20000 kilowatts pour une tonne du 
métal. Elle se déroule en majeure partie dans la cuve d'électrolyse, formée de deux éléments : d'une part, un bloc de carbone, obtenu par cuisson d'un mélange de brai et de coke de pétrole, appelé anode ou électrode positive ; d'autre part, une couche de carbone, constituant le revêtement intérieur de la cuve, obtenue par cuisson d'un mélange de brai et de coke métallurgique. Le deuxième élément de la cuve est appelé cathode ou électrode négative.

Dans cette cuve est versé un électrolyte en fusion, composé de cryolite et de fluorure d'aluminium, auquel on ajoute l'alumine qui s'y dissout. Un courant électrique lancé à travers cette solution la décompose : de l'oxygène se dégage et réagit avec le carbone de l'anode pour donner du gaz carbonique et l'aluminium en fusion se dépose au fond de la cuve. Essentiellement, la fabrication du métal est achevée. Le produit obtenu subira cependant quelques autres transformations mineures avant d'être présenté sur le marché.

Cette description sommaire a permis de prendre connaissance des divers inputs-matières exigés pour la fabrication de l'aluminium. Précisant les aspects techniques de cette production, Ramus a synthétisé ainsi les coefficients d'inputs de l’alumine. Pour obtenir une tonne de ce produit, il faut :

- 2,5 tonnes de bauxite,

- $110 \mathrm{~kg}$ de soude,

- $110 \mathrm{~kg}$ de chaux,

- $450 \mathrm{~kg}$ de fuel,

- 277 kWh d'électricité 56.

Les données suivantes compléteront le tableau. «Une tonne d'aluminium demande pour sa fabrication :

- 1890 d'alumine,

- $450 \mathrm{~kg}$ de carbone, sous forme de coke de pétrole et de coke de brai,

- $50 \mathrm{~kg}$ de cryolite,

- $50 \mathrm{~kg}$ de fluorure d'alumine,

- 17500 kWh d'électricité de basse tension » 57.

56 Y.Ramus, Proposition no 127, article cité, par G. Bell dans «Le pôle électro-métallurgique de Fria », Cahiers de l'ISEA, 1964, p. 46.

57 Ramus et Lerat, « Bauxites et aluminium dans le monde », cité par G. Bell, idem, p. 62. 
De ces données, deux conclusions peuvent être tirées, à savoir que la quantité de matières premières nécessaires à la production de l'aluminium est relativement énorme, le rapport ici étant environ de sept à un, et qu'une usine d'aluminium exige, pour son fonctionnement, d'importantes disponibilités énergétiques, notamment l'électricité. En quoi le Saguenay_Lac-St-Jean pouvait-il répondre à ces contraintes? Plus précisément, pourquoi cette région, faiblement industrialisée, donc faible génératrice d'économies externes, a-t-elle attiré les hommes d'affaires de l'Alcan? Cette question essentielle marquera la prochaine étape de cette étude.

\section{Section 2 : \\ Les facteurs de la localisation de l'Alcan dans le Saguenay-Lac-St-Jean}

$\underline{\text { Retour à la table des matières }}$

Quelles que soient les normes retenues pour en juger, il est rare qu'un site ne présente que des avantages, surtout en ce qui a trait à la localisation d'un complexe industriel. Aussi la présente section comportera-t-elle deux parties. Dans la première, on cherchera à mettre en relief les éléments qui auraient pu constituer des obstacles à l'implantation de l'Alcan dans la région et dans la seconde les facteurs qui ont favorisé cette implantation.

\subsection{Les facteurs défavorables}

On a souligné précédemment que la fabrication de l'aluminium exige un assez fort volume de matières premières. À ce chapitre, le Saguenay_Lac-St-Jean ne se présente nullement comme un milieu idéal. D'autre part, si la proximité d'un marché intéressant constitue une condition favorable à la localisation d'une grande entreprise, l'absence dans la région de puissantes 
agglomérations industrialisées aurait constitué au départ un motif de découragement pour les promoteurs de la Société.

\subsubsection{La délocalisation par rapport aux inputs de base}

Se référant à la première partie de cette étude où a été fait l'inventaire des ressources naturelles de la région, on se rend vite compte que le Saguenay_Lac-St-Jean ne possède aucun des produits de base entrant dans la fabrication de l'aluminium. Cette absence quasi complète des matières premières du métal, à première vue, constitue un facteur absolument défavorable à toute implantation d'une industrie d'aluminium. Ou, tout au moins, la localisation d'une telle industrie dans la région serait appréciée en termes d'importantes différences de rentabilité. Il est vrai que dans cette dernière hypothèse l'entreprise devrait supporter d'énormes frais de transport, à la mesure des distances la séparant de ses sources de matières premières. Or, souligne P. Claval, «les problèmes de frais de transport, de facilité de desserte de localisation... sont primordiaux pour tous les fabricants de produits lourds »58. Le problème de l'implantation de l'Alcan dans le Saguenay-Lac-St-Jean présentait un caractère d'autant plus aigu que la région n'avait pas un réel débouché à offrir à l'industrie d'aluminium.

\subsubsection{L'étroitesse du marché et la main-d'œuvre}

La recherche d'un marché capable d'absorber la production d'une entreprise rentre dans les préoccupations normales de l'entrepreneur en quête d'un milieu d'installation. Mais, un marché large et en expansion régulière suppose l'existence au sein de la région visée d'un certain degré d'industrialisation. De ce point de vue encore, le Saguenay-Lac-St-Jean n'était pas particulièrement apte à accueillir l'Alcan.

En effet, comme il a été déjà souligné, la région était largement sous-industrialisée, à la veille de l'implantation de l'Alcan. L'utilisation du bois dans les scieries d'abord, dans les

58 P. Claval, op. cit., p. 412. 
pulperies et les papeteries ensuite, constituait, en 1925, la très simple formule de l'industrie saguenayenne. En 1924, la région, avec ses neuf usines papetières, pouvait produire jusqu'à 575 tonnes par jour de papier journal, de carton et autres produits connexes. La faiblesse de l'industrie locale et le caractère peu diversifié de l'activité économique sont à mettre en rapport avec l'étroitesse du marché. La population de la région, vers 1925, s'élevait à 100000 habitants environ. La main-d'œuvre atteignait alors à peine 28000 personnes.

Ces chiffres traduisent l'exiguïté du marché régional. Cette population cependant se concentrait sur un espace relativement réduit. En effet, sur l'ensemble de la superficie de la région, «les parties qui étaient alors habitées de façon permanente et divisées en municipalités représentaient une superficie de 2436 milles carrés » 59. Certains auteurs pensent que cette population était très urbanisée, au moins en ce qui concerne le comté de Chicoutimi 60. On a déjà souligné combien il faut être prudent à l'égard de ces observations. On pourra à cet effet considérer le tableau suivant 61.

Comme on peut le voir, le caractère d'urbanisation de la région n'est pas aussi élevé qu'on voudrait le faire croire. Du reste, il est de peu d'intérêt de débattre ici du caractère urbain ou rural de la population régionale. Dans un premier temps, tout au moins, ce sont les industries de la région qui auraient pu servir de débouché à la production d'aluminium de l'Alcan. Or, on a déjà montré que l'industrialisation du Saguenay_Lac-St-Jean était peu poussée vers 1925. Les bases de l'activité économique régionale à cette époque ne permettaient donc pas la création de ces économies externes qui auraient pu attirer une industrie d'aluminium.

59 Statistiques municipales, Québec, 1930.

60 P.-Y. Pépin, op. cit., p. 83.

61 Tableau 7, page suivante. 
Tableau 7

Population agglomérée, région, 1931

\begin{tabular}{|c|c|c|c|}
\hline Comté Chicoutimi & Comté Chicoutimi & Comté du Lac St-Jean & Comté du Lac St-Jean \\
\hline Localités & Population & Localités & Population \\
\hline Chicoutimi (agglo.) & 11877 & Alma & 3970 \\
\hline Jonquière & 9448 & Roberval & 2770 \\
\hline Kénogami & 4500 & Dolbeau & 2032 \\
\hline Bagotville & 2468 & St-Félicien & 1599 \\
\hline Port-Alfred & 2342 & ST-Jérome & 1235 \\
\hline Arvida & 1790 & Mistassini & 970 \\
\hline St-Alexis & 1790 & N.-D. d'Hébertville & 933 \\
\hline Chicoutimi-Nord & 1102 & Hébertville Station & 922 \\
\hline Rivière-du-Moulin & 1040 & Normandin & 773 \\
\hline Laterrière & 529 & Lac-Bouchette & 746 \\
\hline \multirow[t]{8}{*}{ St-Ambroise } & 375 & St-Gédéon & 658 \\
\hline & & Desbiens & 646 \\
\hline & & S.-Cour de Marie & 535 \\
\hline & & Isle-maligne & 449 \\
\hline & & St-Bruno & 426 \\
\hline & & St-Prime & 370 \\
\hline & & Albanel & 329 \\
\hline & & Riverbend & 188 \\
\hline Total & 37261 & Total & 18551 \\
\hline Total général & \multicolumn{3}{|c|}{45812} \\
\hline
\end{tabular}

Source :BFS, Canada, cité par P.-Y. Pépin : op. cit., p. 83.

Il importe d'ajouter, à côté de ce handicap, que la main-d'œuvre régionale ne présentait pas un haut niveau de qualification professionnelle. Ainsi, comme source d'approvisionnement en matière premières, comme réservoir de main-d'œuvre techniquement qualifiée, comme débouché pour l'aluminium, le Saguenay—Lac-St-Jean souffrait de singulières carences. Mais, «les avantages que présente le type d'économie urbaine, notamment en matière de débouchés, sont directement 
fonction de la nature même des productions » 62. Ces avantages sont d'autant plus importants que les productions sont «banales », c'est-à-dire s'adressent au consommateur final. Tel n'est pas le cas de l'aluminium, production «spécifique », susceptible de répondre à une demande déterminée, dispersée dans l'espace. Ces considérations de J. Le Pas incitent donc à admettre que les inconvénients mis en évidence plus haut pouvaient être palliés. D'autant plus que dans le cas concret de la région étudiée des éléments positifs militaient en faveur de l'industrie d'aluminium.

\subsection{Les facteurs favorables}

$\underline{\text { Retour à la table des matières }}$

Délocalisé par rapport aux matières premières, éloigné des grands marchés, un complexe de fabrication d'aluminium, pour s'installer au Saguenay-Lac-St-Jean, avait besoin, de manière vitale, de voies et moyens adéquats de liaison avec l'extérieur. D'un autre côté, l'examen des conditions techniques de la production d'aluminium a révélé que cette industrie est grosse consommatrice d'électricité. Or, le moyen le plus rentable de produire l'électricité reste, encore aujourd'hui, la force hydraulique. Et, à la différence de certaines matières premières qui peuvent être transportées sur de longues distances à un coût acceptable, « l'électricité doit être utilisée près de sa source. » Cela implique la nécessité pour « toute grande usine d'aluminium de s'établir à proximité d'une source abondante d'énergie hydroélectrique » 63.

De plus, l'électricité constituant une portion importante du prix de revient de l'aluminium, il est préférable d'implanter l'usine consommatrice d'énergie loin des grands centres où les autres industries, pour lesquelles l'électricité intervient moins fortement dans le coût de production peuvent surenchérir pour l'achat de l'électricité disponible, alors que celle de l'aluminium ne le pourrait pas. À ce dernier égard, le Saguenay-Lac-St-Jean, où le climat a su s'allier au relief pour lui donner de vastes ressources hydrauliques, s'avérait être un site idéal. Mais, il importe d'examiner de

62 J. Le Pas, op. cit., p.1269.

63 Mc Guire et Sarrazin, « Aluminium, histoire de l’Alcan », traduction française, Canadian Geographical Journal, 1963, p. 6. 
plus près le double problème des conditions de transport et de la production hydroélectrique dans la région.

\subsection{Les facilités de transport}

Dans les années 1920, on peut admettre que la région était sortie de la période héroïque de la «colonisation», caractérisée par son excentricité et son isolement. Trois aspects retiendront l'attention : la navigation, les voies ferrées et le système routier.

\subsubsection{Navigation et ports}

Dans ce domaine, tout reposait et repose encore aujourd'hui sur l'émissaire du St-Laurent, « la voie royale du Saguenay ». Cette voie d'eau, déjà connue des Indiens qui la remontaient sur leurs canoës durant la belle saison, constituait le cordon ombilical des premiers explorateurs et des colons. Ses profondeurs les plus faibles, à marée basse, dépassent vingt pieds. Aussi est-elle navigable sur la plus grande partie de sa longueur, soit plus de 110 kilomètres, de son point de jonction avec le St-Laurent, à la hauteur de Tadoussac, jusqu'à la ville de Chicoutimi. Déjà en 1842, un bateau à vapeur avait atteint le port de Chicoutimi, ce qui cependant était considéré à l'époque comme une aventure. Mais, huit ans plus tard, la région commença à être régulièrement desservie par des bateaux à vapeur, d'abord jusqu'à la Baie Ha ! Ha ! ( PortAlfred), puis jusqu'à Chicoutimi, en 1870. La rivière Saguenay offrait ainsi une excellente voie d'entrée pour les matières premières nécessaires aux éventuelles usines de l'Alcan et de sortie pour les lingots d'aluminium. En outre, deux ports fonctionnaient déjà : celui de Chicoutimi et celui de Port-Alfred.

Le port de Chicoutimi, le plus ancien, constitue la limite de la partie navigable du Saguenay. Ses quais, parallèles à la rivière, mesurent près de 2500 pieds de long, ce qui permet au port d'accueillir plusieurs grands bateaux simultanément. Pourtant, il reste de 
loin moins important que celui de Port-Alfred, « création purement commerciale, destinée à ravitailler les industries en matières premières et en combustibles » 64.

Construits et aménagés, vers 1915, par la Société d'exploitation du port de Chicoutimi, The Port of Chicoutimi Ltd, les premiers quais de Port-Alfred ne mesuraient pas moins de 2 500 pieds et permettaient l'accostage simultané de plusieurs navires. Il présentait un avantage sur le port de Chicoutimi. En effet, construit au centre de la Baie Ha ! На !, ce port, large de plus de trois kilomètres, pouvait abriter des centaines de bateaux. À quai, les profondeurs de la rivière y atteignent trente pieds et s'accroissent très rapidement à faible distance de la berge. La navigation de haute mer s'y trouvait favorisée.

À ces avantages s'alliait toutefois un inconvénient majeur : la cessation des activités de la navigation durant la période relativement longue de l'hiver, où la rivière est prise dans les glaces. Mais, à ce problème, une première solution pouvait être trouvée dans l'entreposage, puisque les matières premières de l'aluminium ne sont pas périssables. Par ailleurs, c'est le propre des entrepreneurs dynamiques de miser sur les progrès techniques. En ce sens, l'avenir allait donner raison aux promoteurs de l'Alcan, car souligne M. Brochu, « ouvrir le Fjord Saguenay à la navigation d'hiver en 1967 n'est plus une entreprise téméraire, ni même audacieuse. C'est purement et simplement une technique très simple » 65. En effet, poursuit l'auteur, « il suffirait de donner dès le début de l'hiver un coup de butoir dans la nouvelle glace en voie de formation du Saguenay, puis ensuite, environ une fois par semaine, pour que ce fjord reste navigable sans difficulté tout l'hiver » 66.

\subsubsection{Les voies ferrées}

64 B. Brouillette et P. Dagenais, "Quelques aspects de l'économie du Saguenay-Lac-St-Jean », dans L’Actualité économique, janvier 1948, p. 680.

65 M. Brochu, «Les grands objectifs de la navigation d'hiver au Québec », dans L'Actualité économique, no 2, juillet-septembre 1967, pp. 358-359.

66 M. Brochu, idem, p. 359. 
Aux facilités qu'offraient le Saguenay et ses deux ports s'ajoutaient celles des voies ferrées. Déjà, en 1888, une ligne reliait la capitale provinciale à Roberval et à Chambord. Cette ligne devait se prolonger jusqu'à Chicoutimi, en 1893, puis à Bagotville, en 1910. Par des adjonctions successives, le réseau de voies ferrées de la région se développa. Le tableau 8 résume les principales phases de la mise en place du système régional de chemins de fer.

Plus de 75\% du réseau régional de voies ferrées était donc en place avant l'implantation de l'Alcan. Les différentes lignes appartiennent aujourd'hui aux Chemins de fer Fédéraux à l'exception de celles qui sont exploitées pour le compte de l'Alcan par les Sociétés Roberval and Saguenay et Alma and Jonquière.

Tableau 8

\section{Chronologie de la construction des lignes de chemin de fer du Saguenay-Lac-St-Jean}

\begin{tabular}{|c|c|c|}
\hline \multicolumn{1}{|c|}{ Ligne } & Année & $\begin{array}{c}\text { Longueur } \\
\text { (milles) }\end{array}$ \\
\hline Quebec and Lake St-John Ry (1) & & \\
\hline - Québec à Roberval & 1888 & 191,0 \\
\hline - Chambord-Chicoutimi & 1894 & 51,2 \\
\hline James Bay and Eastern Ry (1) & & \\
\hline - Roberval-St-Félicien & 1910 & 17,6 \\
\hline Roberval and Saguenay Ry (1) & & \\
\hline - Jonction Baie Ha ha (Arvida) à Bagotville & 1910 & 18,0 \\
\hline - Labrosse-Chicoutimi & 1912 & 3,4 \\
\hline - Chicoutimi-Laterrière & 1912 & 8,0 \\
\hline - Jutres embranchements & 1913 & 4,0 \\
\hline Alma and Jonquière Ry (1) & 1926 & 5,0 \\
\hline ' Hébertville-Isle-Maligne & & \\
\hline Canadian National Ry (1) & 1923 & 11,5 \\
\hline - St-Félicien-Dolbeau & & \\
\hline Total $\quad 1930$ & 28,0 \\
\hline
\end{tabular}

(1) Compagnie d'exploitation de la ligne.

Source : B. Brouillette et P. Dagenais : op. cit., p. 682. 


\subsubsection{Le réseau routier}

Pour les activités de l'Alcan, les voies terrestres s'avéraient relativement de peu d'importance. En effet, le caractère pondéreux de l'aluminium et des matières premières entrant dans sa fabrication rend le coût de transport par la route sinon prohibitif du moins très élevé. Mais, même indirectement, la présence d'un réseau routier convenable présentait un intérêt certain pour la localisation des usines de la Compagnie.

Selon P.-Y. Pépin, « la première automobile fut introduite dans la région vers 1905 ; à cette époque, le camionnage était à un stade plus qu'artisanal de développement » 67. On peut penser que cette situation était déjà dépassée en 1925. En effet, « depuis l'après-guerre 1914-18, le Québec s'était orienté vers l'amélioration de son réseau routier, tant pour répondre aux besoins régionaux que pour promouvoir le tourisme » 68. De fait, déjà, plusieurs routes de grande circulation reliaient la région au reste de la Province, telle, via le Parc des Laurentides, la route Québec - Hébertville, d'une longueur de plus de deux cents kilomètres. À l'intérieur, le Tour du Lac St-Jean ne comptait pas moins de 240 kilomètres, reliant entre elles les différentes localités situées sur le bord du Lac St-Jean. Dans l'ensemble, le réseau routier de la région totalisait plus de mille kilomètres.

Les conditions des transports régionaux compensaient largement la délocalisation d'éventuelles usines d'aluminium par rapport à leurs sources de matières premières et à leur marché. Mais, le facteur primordial de l'implantation de la Compagnie Alcan dans le Saguenay—Lac-St-Jean était l'abondance du potentiel des forces hydroélectriques.

67 P.-Y. Pépin, op. cit., p.89.

68 B. Brouillette et P. Dagenais, op. cit., p. 683. 


\subsection{Les forces hydroélectriques}

Au cours de la première partie de cette étude, on a fait état des immenses potentialités de la région dans le domaine des forces hydroélectriques. Le Saguenay-Lac-St-Jean, de l'avis de tous les observateurs, « forme un bassin de drainage remarquable par son étendue et sa puissance en sources d'énergie hydraulique » 69. Au total, le bassin hydrographique de la Rivière Saguenay couvre une superficie de 30000 milles carrés. Mais, il a été souligné que « jusqu'en 1925, ces vastes ressources étaient demeurées à peu près inexploitées » 70.

C'est que le régime nival qui caractérise les cours d'eau de la région en rend l'écoulement très irrégulier. Pour ne citer qu'un exemple, « les jaugeages effectués par la Commission des Eaux courantes du Gouvernement du Québec de 1913 à 1915 montrèrent que si le plus fort débit observé du Saguenay (11-12 mai 1913) était de 1370000 gallons (6 228 mètres cubes), ce débit pouvait tomber à la fin de l'hiver (avril 1915) à 7500 gallons (340 mètres cubes), soit 18 fois moins » 71. Pour tirer parti des ressources offertes par le bassin hydrographique du Saguenay, il fallait procéder à la régularisation préalable des cours d'eau.

Dès le début du XXe siècle, des entrepreneurs, appuyés par le Gouvernement de la Province, se lancèrent dans la construction de barrages pour régulariser les eaux du Lac Kénogami. Ces travaux furent complétés entre 1923 et 1925. Le niveau du lac fut relevé de trente-deux pieds et sa capacité d'emmagasinement, portée à 382 millions de mètres cubes. Ces résultats garantissaient un débit annuel régulier de 375 et 750 gallons respectivement pour les rivières Chicoutimi et Aux Sables. Dans le même temps, on entreprit de transformer le Lac St-Jean en un véritable réservoir. Une Société fut constituée, dont les principaux promoteurs étaient James Duke, le roi américain du tabac, et W. Price. Le premier était à la recherche de sources d'énergie pour la fabrication d'engrais chimiques azotés et le second, industriel déjà connu dans la région, voulait étendre les activités de ses usines papetières.

69 B. Brouillette et P. Dagenais, op. cit., p. 670.

70 Alcan, L'énergie électrique au service de l'aluminium, p. 3.

71 R. Blanchard, L’Est du Canada français, op. cit., p. 99. 
Les travaux débutèrent en 1923, sous limpulsion de la nouvelle Société, le Saguenay Development Company, créée une année auparavant. Par un barrage élevé à sa sortie, le niveau du Lac St-Jean enregistra une augmentation de 17,5 pieds. En outre, huit barrages, dont sept en béton, furent construits, dotés de vannes capables de déverser plus de 13000 mètres cubes d'eau à la seconde. Mais, de ce vaste programme, l'érection de la centrale d'Isle-Maligne constituait l'élément le plus intéressant pour les projets de l'Alcan.

En effet, la capacité prévue de cette centrale dépassait 525000 ch.-vap., soit 400000 KWh environ, ce qui ne représentait, somme toute, qu'un faible pourcentage des potentialités hydrauliques régionales, en attente d'être réalisées. Ces travaux prirent fin en 1925. Cependant, déjà avant cette date, l'Alcan avait opté pour son implantation dans la région. Les facteurs favorables avaient emporté l'adhésion.

\section{Section 3 : L'implantation}

\subsection{Les préalables}

\section{$\underline{\text { Retour à la table des matières }}$}

L'origine de l'implantation de l'Alcan dans le Saguenay reste assez curieuse 72. L'histoire des négociations qui y ont abouti prouve, si besoin en était, combien a été sévère la lutte entre les industriels pour la conquête des importantes sources d'énergie de la région. Comme l'a noté D. H. Wallace, « en dépit de la croissance accrue de la demande d'aluminium qui avait porté cette industrie au premier rang des industries métalliques, aucune nouvelle entreprise d'importance ne pouvait voir le jour qui ne fût lancée ou protégée par un gouvernement ou des firmes anciennes déjà solidement implantées » 73.

72 Pour une vue plus complète de cette question, on peut voir D. H. Wallace, Market Control in the Aluminium Industry, Cambridge University Press, 1937, p. 132 et ss.

73 D. H. Wallace, idem, p. 129. 
La concurrence fut d'autant plus âpre que le coût de l'énergie du Saguenay était relativement bas. En effet, le cheval-vapeur annuel de la région était estimé à cinq dollars environ pour un investissement de base de 65 dollars, alors qu'un investissement moyen de deux cents dollars, estimaient les experts de l'Aluminum Company of America était nécessaire pour l'obtention d'un cheval-vapeur aux États-Unis. Ainsi, on comprend que plus d'un compétiteur s'étaient présentés pour être acquéreurs des importantes sources d'énergie du Saguenay-Lac-St-Jean.

Les projets de Duke, de création d'une usine de fabrication d'engrais chimiques azotés, avaient échoué. Principal intéressé dans la construction de la centrale de l'Isle-Maligne, il devenait un fournisseur potentiel d'énergie électrique. Vers 1924, il fut mis en contact avec un industriel américain, G.D.Haskell, président de la Bausch Machine Tools Company de Massachussets. Fabricant de duralumin, Haskell projetait de produire lui-même l'aluminium nécessaire au fonctionnement de son entreprise et secouer ainsi la tutelle de son principal fournisseur, l'Aluminum Company of America (Alcoa). Mais, cette dernière société s'intéressait également à l'énergie d'Isle-Maligne. Elle avait, à l'insu de Haskell, entamé des négociations avec Duke. Dans cette compétition, l'Alcoa présentait un triple avantage sur son principal concurrent.

Tout d'abord, elle était mieux connue au Québec, où, par l'intermédiaire d'une de ses filiales, la Northern Aluminum Company, elle avait installé dès 1900 les premières usines d'aluminium, à environ trente milles au nord de la ville de Trois-Rivières. Ces usines avaient bien fonctionné et constituaient un apport relativement important à l'économie de cette région. Deuxièmement, l'Alcoa était financièrement beaucoup plus puissante que son adversaire. Elle contrôlait déjà la majeure partie de la production d'aluminium des États-Unis et du Canada. À ce titre, Pitaval a noté que «l'histoire de l'aluminium aux États-Unis et au Canada est en somme celle de l'Aluminum Company of America... En 1939, l'Alcoa venait en tête des quatre principales entreprises des États-Unis, dont le capital contrôlait 44\% de la production mondiale de bauxite et 52\% de celle de l'aluminium » 74. Enfin, troisième avantage et non le moindre, l'Alcoa pouvait se prévaloir d'un accord passé avec la Grande-Bretagne pour se faire considérer comme acheteur privilégié. Dès

74 R. Pitaval, Histoire de l'aluminium, métal de la victoire, Publications minières et métallurgiques, Paris, 1946, pp. 147-148. 
après la première guerre mondiale, elle avait obtenu une importante concession de bauxite sur les gisements de la Guyane britannique. Le contrat avait été ratifié sous la condition explicite que l'usine d'aluminium qui utiliserait cette bauxite soit installée sur les territoires de l'Empire. Le moment était venu de mettre cette clause en application et obtenir le droit d'implantation dans cette partie du Dominion Canadien qu'est la région.

Forte de ces trois atouts, l'Alcoa n'eut pas de peine à évincer son rival, Haskell. De fait, en juin 1925, un accord fut passé avec la Société Duke-Price qui assurait à l'Alcoa les droits sur la Chute-à-Caron et la Rivière Saguenay, jusqu'à son point de confluence avec le Shipshaw. En outre, une quantité de 100000 chevaux-vapeur de la centrale de l'Isle-Maligne était mise annuellement à la disposition des entreprises futures de l'Alcoa. Désormais, la voie était ouverte à l'installation des usines d'aluminium au Saguenay-Lac-St-Jean. L'Alcoa avait déjà dépensé dans cette première phase des opérations environ 16 millions de dollars.

\subsection{Les installations}

$\underline{\text { Retour à la table des matières }}$

La décision concernant le site de la nouvelle usine fut rapide : une vaste terrasse d'argile, située entre Kénogami et la ville de Chicoutimi, non loin des Chutes-à-Caron et du Shipshaw. Ce choix comportait le double avantage de la proximité des sources d'énergie et des possibilités importantes en vue d'éventuels agrandissements. On sait en effet que le transport à longue distance de l'électricité, même sur des lignes à haute tension, donne lieu à une déperdition coûteuse. D'autre part, il fallait prévoir les conjonctures où la Société aurait à augmenter rapidement sa production. Il est vrai que le marché mondial était alors relativement déprimé. Entre 1919 et 1922, la production mondiale avait baissé de plus de moitié, passant de 200000 tonnes à 100000 tonnes d'aluminium brut. Mais, pour les industriels, la progression de la consommation mondiale d'aluminium était irréversible. A.V. Davis, alors président de l'Alcoa, avait noté que la première guerre mondiale « gave a 
tremendous impetus to the aluminum industry » 75. Les espoirs dans une éventuelle expansion des activités étaient donc légitimes.

Pourtant, encore aujourd'hui, le choix de ce site continue à susciter les critiques de certains observateurs. On se réfère généralement à la distance qui sépare le site de l'usine et le point d'arrivée des ses matières premières, Port-Alfred. «On ne peut s'empêcher de penser, souligne Blanchard, que le choix de l'emplacement n'était pas particulièrement heureux. ... Grosse mangeuse de matières premières,... l'usine d'aluminium, installée au fond de la Baie Ha ! Ha !, à 19 milles plus à l'Est, se fût épargnée des transports onéreux » 76. Cette critique ne semble pas justifiée, car si on en croit Pitaval, « l'aluminium étant un métal léger et relativement cher, cette question de transport ne joue pas le même rôle que pour d'autres produits métallurgiques » 77 . Du reste, les 19 milles, dont fait état Blanchard, représentent un pourcentage relativement minime par rapport au parcours total que doivent emprunter les matières premières nécessaires au fonctionnement de l'usine. Par ailleurs, « si l'usine de 1926 se fût installée sur la rive de la Baie Ha ! Ha !, elle aurait dû rechercher à plusieurs milles à l'intérieur l'espace aplani suffisant pour s'agrandir plus tard, en $1940 » 78$.

Dès le milieu de 1925, les chantiers de l'Alcoa purent s'ouvrir, avec les milliers d'ouvriers qui avaient participé aux travaux d'aménagement du Lac St-Jean et à la construction de la centrale d'Isle-Maligne. Il s'agissait de mettre en place les installations pour la nouvelle usine d'aluminium et de créer les centres d'accueil et les logements nécessaires à la main-d’œuvre. Les opérations furent menées avec rapidité, puisque un an plus tard, en juillet 1926, la nouvelle usine put commencer ses activités de production. Elle ne fut achevée, cependant, sous sa forme définitive, qu'en 1927. Sa capacité de production était alors d'une trentaine de milliers de tonnes de lingots d'aluminium par an.

75 R. Pitaval, op. cit., p. 116.

76 R. Blanchard, L’Est du Canada français, op. cit., p. 105.

77 R. Pitival, op. cit., p. 125.

78 B. Brouillette, L'aluminium au Saguenay, op. cit., p. 419. 
Simultanément, les travaux de construction d'une véritable ville se poursuivaient. La nouvelle cité, ARVIDA, des premières syllabes du nom du président de l'Alcoa, Arthur Vining Davis, était une ville modèle, dont les plans avaient été conçus et mis en oeuvre par des ingénieurs et des architectes embauchés par la Compagnie 79. À l'origine, elle pouvait accueillir 3000 personnes, mais des possibilités d'agrandissement étaient prévues pour en faire une ville d'une trentaine de milliers d'habitants.

Pour l'économie du Saguenay—Lac-St-Jean, l'implantation de cette usine d'aluminium marquait une étape nouvelle de son évolution. En effet, jusque-là, l'exploitation des ressources forestières avaient constitué l'essentiel de l'industrialisation régionale. On pouvait désormais miser sur l'aluminium pour le futur développement économique de la région. Il importe, avant de voir si les espoirs ont été justifiés, de dévoiler la nature véritable de la Société qui venait ainsi de faire son entrée sur la scène de l'économie du Saguenay—Lac-St-Jean.

79 Pour plus de détails sur la fondation de la ville d'Arvida, on peut consulter B. Ellison, « Arvida, a Modern City », dans Engineering and Contract Record, juillet, 1945. 
Deuxième partie :

L’Alcan et l'évolution de l'économie du S.-L.-S-.J.

\section{Chapitre IV}

\section{La triple nature d’Alcan}

$\underline{\text { Retour à la table des matières }}$

L'Alcan, société aux visages multiples, dont les activités s'étendent à maints domaines et se déroulent dans toutes les parties du monde, est de nature fort complexe. Par exemple, le lecteur a pu croire à une certaine confusion, quand, traitant de l'implantation, on a parlé de l'Alcoa (Aluminum Company of America). Le présent chapitre tâchera de lever cette inévitable ambiguïté, en présentant l'Alcan sous sa triple dimension, de société multinationale, de compagnie canadienne et d'entreprise régionale. D'où les trois sections de ce chapitre.

\section{Section 1 : Alcan, grande unité inter-territoriale}

Par les moyens mis en oeuvre, la dimension de ses activités et la place qu'elle occupe dans l'industrie mondiale d'aluminium, l'Alcan est une grande unité de production, au sens que le Professeur Byé donne à cette expression, « un ensemble organisé de moyens soumis à un centre unique, capable d'autonomie à l'égard du marché » 80. Elle est multinationale,

80 M. Byé, « La grande unité interterritoriale », dans Revue d’Économie politique, mai-juin 1957, p. 271. 
car « ses moyens de production sont dispersés sur plusieurs territoires et servent le marché mondial » 81.

\subsection{Les origines de l’Alcan}

$\underline{\text { Retour à la table des matières }}$

La formation même de l'Alcan procède de la stratégie de grande unité inter-territoriale. En effet, l'Alcan, lors de son incorporation en 1902 sous le nom de Northern Aluminum Company, Limited, était une filiale de l'Alcoa, société américaine, fondée à Pittsburg, en 1887. Par « une politique fort habile qui prenait souvent l'apparence humanitaire de sauvetage d'entreprises malheureuse », l'Alcoa était devenue la principale entreprise productrice d'aluminium des États-Unis. Aussi n'eut-elle pas de peine à s'implanter au Canada et dans diverses autres parties du monde. Les participations et filiales de l'Alcoa à l'étranger étaient devenues tellement nombreuses qu'elle décida de les passer à une société de holding canadienne créée à cet effet, l'Aluminium Limitée. La Northern Aluminum Company, devenue Aluminum Company of Canada Limited en juillet 1925, était la principale filiale du nouvel holding. Au début, cette société n'avait de canadienne que le nom. En effet, « le 4 juin 1928, l'Aluminum Limited, constituée au Canada quelques jours auparavant, émit 490895 actions ordinaires qui furent toutes distribuées, sauf vingt actions des fondateurs, aux actionnaires de l'Aluminum Company of America » 82.

Le caractère des liens qui unissaient les deux sociétés, à l'origine, ajouté au fait que le président de l'Aluminium of Canada Limited, E. K. Davis, fut le frère du président de l'Alcoa, fit dire à Wallace que «the two companies were brotherly in other respects as well » 83. Il semble que, très tôt, ces liens se distendirent et que, octroyée ou acquise, l'Alcan en vint à avoir une certaine autonomie. Un jugement de la Cour d'Appel des États-Unis, le 12 mars 1945, proclamait en effet que « l'Aluminium Limited du Canada n'avait pas été créée par l'Alcoa pour lui servir d'agent,

81 M. Byé, idem, pp. 273-274.

82 B. Brouillette, « L’aluminium dans le Saguenay », op. cit., p. 420.

83 H. D. Wallace, op. cit., p. 106. 
qu'elle n'était pas sous le contrôle financier de l'Aluminum Company of America » 84. On est loin aujourd'hui de l'époque où l'indépendance financière de l'Alcan constituait le thème de débats passionnés. À toutes fins pratiques, les responsables de la Société soulignent à l'attention de tous les intéressés que «l'Alcan Aluminium Limitée est une entreprise canadienne dont le siège social est à Montréal... Elle est indépendante de tous les autres grands producteurs mondiaux d'aluminium et leur fait concurrence sur tous les marchés » 85 . Cette entreprise serait d'autant plus canadienne que ses actions ordinaires, au 31 décembre 1972, étaient détenues en majorité (55\%) par des personnes résidant au Canada, ainsi que la presque totalité des actions privilégiées. Quoiquil en soit, personne ne peut mettre aujourd'hui en doute l'importance vitale de l'Alcan pour l'économie canadienne, en général, et celle du Saguenay—Lac-St-Jean, en particulier, malgré son caractère indiscutable de grande entreprise multinationale.

\subsection{L'activité industrielle de l'Alcan}

\section{$\underline{\text { Retour à la table des matières }}$}

Déjà à ses débuts, et pour cause, l'Aluminium du Canada Limitée faisait figure de grande entreprise. Son actif était alors estimé à dix millions de dollars et, en 1929, année relativement défavorable à cause des effets de la grande crise, les bénéfices enregistrés de la Société atteignaient 2 millions de dollars. Mais, rapidement, ces chiffres-témoins allaient se multiplier. Évalué au prix coûtant, l'actif fixe de l'Alcan passait à 328,7 millions de dollars en 1944 et les profits nets à 12,1 millions 86. Un an plus tard, les mêmes variables atteignaient respectivement 768,4 et 33,1 millions de dollars 87. Aujourd'hui, la puissance économique et financière de cette société se reflète à travers ses multiples activités de production, s'étendant à l'extraction de la bauxite à la transformation des lingots

84 B. Brouillette, op. cit., p. 421.

85 Alcan, Précis Alcan 1969, p. 2.

86 Financial Statements, Aluminium Company of Canada, Limited, 31st December 1944.

87 Financial Statements, Aluminium Company of Canada, Limited, 31st December 1945. 
d'aluminium en produits finis. L'espace économique de l'Alcan est le globe terrestre, car il n'y a pas un seul continent où la Compagnie n'ait réussi à s'implanter.

\subsubsection{Les matières premières}

Par ses nombreuses filiales et participations, l'Alcan Aluminium Limitée possède d'immenses réserves de bauxite à travers le monde. D'après les experts de la Société, ces réserves sont de nature à répondre aux besoins des usines d'électrolyse de l'Alcan pendant plus d'un demi-siècle. C'est dire que la Compagnie est largement autonome face au marché, en ce qui concerne son approvisionnement en matières premières. En dépit de la nationalisation récente, par le Gouvernement, de la Demerara Bauxite Company, Ltd, filiale de l'Alcan qui faisait l'extraction de la bauxite des importants gisements de Guyane et produisait de l'alumine, les concessions appartenant encore à l'Alcan sont énormes, ainsi qu'on peut le voir à travers le tableau suivant.

Tableau 9

Les usines d'alumine de l'Alcan, 1972

\begin{tabular}{|l|l|c|c|}
\hline Pays & Usines & $\begin{array}{c}\text { Pourcentage de } \\
\text { participation }\end{array}$ & $\begin{array}{c}\text { Capacité nominale } \\
\text { Annuelle (1) }\end{array}$ \\
\hline Canada & Arvida & 100 & 1387 \\
\hline Jamaïque & Kirkvine & 100 & 613 \\
\hline & Ewarton & 100 & 613 \\
\hline Inde & Belgaum & 65 & 103 \\
\hline & Muri & 65 & 85 \\
\hline Brésil & Saramenha & 100 & 66 \\
\hline Total des filiales & & & 2867 \\
\hline Australie & Gladstone & 22 & 1428 \\
\hline Japon & Shimizu & 50 & 577 \\
\hline & Tomakomaï & 50 & 353 \\
\hline Total participations & & & 5224 \\
\hline Total général & & & \\
\hline
\end{tabular}

(1) Exprimée en milliers de tonnes, avril 1972.

Source : Précis Alcan 1972. 
Si on se rappelle qu'il faut environ 2,5 tonnes de bauxite pour une tonne d'alumine, on conclut par simple calcul que les entreprises de l'Alcan pouvaient extraire plus de dix millions de tonnes de bauxite en 1972. Or, la Société participe encore à d'autres consortiums, dont les plans d'exploitation sont projetés pour un futur très rapproché. C'est ainsi qu'elle détient 27\% des parts du consortium qui mettra en valeur les gisements de bauxite de Boké, en République de Guinée. La capacité initiale prévue de cette entreprise, dont les opérations débuteront au deuxième semestre de 1973, sera de 6 millions de tonnes environ. La fabrication de l'aluminium exige d'autres matières premières essentielles, comme le coke de pétrole, la cryolite et le spath fluor. En ce qui concerne la première, l'Alcan s'approvisionne au Canada et aux États-Unis. Elle procède ellemême à la fabrication de la cryolite artificielle et achète de la cryolite naturelle au Groenland. La fluorine, dont on tire le fluorure d'aluminium, lui provient de ses propres exploitations de TerreNeuve, au Canada, et elle en possède en outre des gisements au Mexique. Quant aux ressources énergétiques, la Société possède ses propres centrales hydroélectriques dont la puissance est de

plus de 3,5 millions de KW au Canada. À l'étranger, les 50\% des besoins des usines de l'Alcan sont satisfaits par ses propres centrales, le reste étant laissé aux soins d'autres établissements, comme en France. Mais, même dans ce dernier cas, la Compagnie est garantie de contrats d'approvisionnement à long terme.

\subsubsection{L'aluminium}

Indépendante du marché pour les matières premières, l'Alcan a développé sa production d'aluminium sur une très grande échelle. Elle possède treize usines d'aluminium de première fusion et participe à de très nombreuses réparties à travers le monde. « En décembre 1972, la capacité nominale annuelle de l'ensemble des usines, dont elle assure elle-même l'exploitation, s'élevait à 1360000 tonnes (y compris 1035000 tonnes au Canada) ». Le tableau qui suit donne la mesure de la puissance de l'Alcan dans le domaine de l'électrolyse de l'aluminium. 
Tableau 10

Alcan : les usines d'aluminium

\begin{tabular}{|c|c|c|c|}
\hline Pays & Usines & $\begin{array}{c}\% \text { de participation } \\
\text { d'Alcan }\end{array}$ & $\begin{array}{c}\text { Capacité annuelle } \\
\text { en tonnes }\end{array}$ \\
\hline \multirow[t]{6}{*}{ Canada } & Arvida & 100 & 458500 \\
\hline & Isle-maligne & 100 & 130000 \\
\hline & Shawinigan & 100 & 95000 \\
\hline & Beauharnois & 100 & 51500 \\
\hline & Kitimat & 100 & 300000 \\
\hline & & & 1035000 \\
\hline \multirow[t]{2}{*}{ Brésil } & Saramenha & 100 & 30000 \\
\hline & Aratu & 100 & 11000 \\
\hline Royaume-Uni & Lynemouth & 100 & 132000 \\
\hline Italie & Borgofranco d'ivrea & 100 & 4000 \\
\hline \multirow[t]{3}{*}{ Inde } & Bealgaum & 65 & 52000 \\
\hline & Hirakud & 65 & 25000 \\
\hline & Alupuram & 65 & 21000 \\
\hline Australie & Kurri Kurri & 70 & 50000 \\
\hline Total des filiales & & & 1360000 \\
\hline \multirow[t]{5}{*}{ Norvège } & Ardal & 50 & 193000 \\
\hline & Sunndal & 50 & 132000 \\
\hline & Hoyanger & 50 & 33000 \\
\hline & Tyssedal & 50 & 29000 \\
\hline & Eydehavn & 50 & 16000 \\
\hline \multirow[t]{2}{*}{ Espagne } & Valladolid & 25 & 26000 \\
\hline & Aviles & 25 & 64000 \\
\hline Suède & Sundsvall & 21 & 95000 \\
\hline \multirow[t]{3}{*}{ Japon } & Kambara & 50 & 123000 \\
\hline & Niigata & 50 & 108000 \\
\hline & Tomakomaï & 50 & 143000 \\
\hline Total participations & & & 962000 \\
\hline Total général & & & 2322000 \\
\hline
\end{tabular}

Source : Alcan, Service des Relations Extérieures, 1972 


\subsubsection{Le secteur de transformation}

Il a été déjà souligné que l'Alcan est une société de production industrielle parfaitement intégrée. Elle participe donc pour un fort pourcentage à la transformation de l'aluminium brut en produits semi-finis et articles de consommation finale.

Jusqu'à la seconde guerre mondiale, l'essentiel des activités de l'entreprise dans ce domaine, se déroulait uniquement dans les pays du Commonwealth, notamment au Canada, en Australie, en Inde et en Grande-Bretagne. Après 1945, les entreprises Alcan de transformation de l'aluminium se répandirent au Mexique, en Afrique du Sud, au Brésil, au Japon et dans la plupart des pays industrialisés d'Europe. L'expansion des activités de la Compagnie dans ce secteur procurait des débouchés sûrs à l'aluminium de première fusion. Il n'est pas question de faire ici l'inventaire, même rapide, des mille utilisations des lingots d'aluminium. De toute manière, l'éventail des produits en aluminium fabriqués par les usines de l'Alcan est très large.

Pour rendre compte de l'importance de ce secteur dans l'ensemble des activités de la Société, on signalera que de 1959 à 1971 plus de 600 millions de dollars y ont été engagés. Pour la seule année 1971, le poids des produits semi-finis ou finis d'aluminium livrés par les filiales de l'Alcan et ses participations atteignait 1027000 tonnes, soit un accroissement d'environ $10 \%$ par rapport à 1970 et $25 \%$ par rapport à 1968. Cette vigoureuse expansion du secteur de transformation n'aurait pas été possible sans le support d'un solide réseau de distribution, à la dimension des activités proprement industrielles de la Compagnie.

\subsubsection{Activité commerciale et structure financière}

2.4.1 L'activité commerciale de l'Alcan a suivi de près le même rythme d'évolution que son activité de production. L'organisation de la vente des produits de la Société reflète donc son caractère de grande unité inter-territoriale. Une filiale spécialisée dont le siège social 
est également à Montréal coiffe cette gigantesque pyramide qui se compose de huit grandes sociétés, chargées de la commercialisation des produits Alcan à travers le monde :

\section{Sociétés de commercialisation}

- Alcan Sales, New-York

- Alcan Aluminio,Ltd, Buenos Aires

- Alcan (U.K.) Ltd, Londres

- Alcan S.A., Zurich

- Alcan Metall GmbH, Francfort

- Alcan Asia Ltd, Osaka

- Alcan Southeast Asia Ltd, Hong-Kong

\section{Territoire}

États-Unis, Antilles

Amérique Latine

Royaume-Uni, Scandinavie

Europe continentale (à l'exception de la Scandinavie et de l'Allemagne), Afrique du Nord et Moyen-Orient

Allemagne

Japon, Afghanistan, Pakistan et certaines régions d'Asie

Hong-Kong, Inde, Philipines et autres régions d'Asie

Source : Alcan Trading Limited, Montréal,1971

Les bureaux de vente, représentants et agents de la Compagnie sont installés dans plus de cent pays. Le marché des produits de l'Alcan coïncide ainsi avec la planète, comme le traduit la distribution géographique des ventes d'aluminium. 
Tableau 11

Alcan Aluminium Limitée

Répartition géographique des ventes d'aluminium

(en milliers de tonnes)

\begin{tabular}{|l|c|c|c|c|c|c|c|}
\hline Territoire & $\mathbf{1 9 6 5}$ & $\mathbf{1 9 6 6}$ & $\mathbf{1 9 6 7}$ & $\mathbf{1 9 6 8}$ & $\mathbf{1 9 6 9}$ & $\mathbf{1 9 7 0}$ & $\mathbf{1 9 7 1}$ \\
\hline Canada & 114 & 137 & 130 & 150 & 152 & 160 & 189 \\
\hline États-Unis & 342 & 395 & 332 & 393 & 399 & 357 & 387 \\
\hline Royaume-Uni & 162 & 360 & 172 & 174 & 191 & 222 & 186 \\
\hline Marché Commun & 83 & 97 & 102 & 121 & 158 & 168 & 148 \\
\hline Autres pays & 292 & 326 & 368 & 382 & 463 & 439 & 488 \\
\hline Total & $\mathbf{9 9 3}$ & $\mathbf{1} \mathbf{1 1 5}$ & $\mathbf{1 1 0 4}$ & $\mathbf{1} \mathbf{2 2 0}$ & $\mathbf{1} \mathbf{3 6 3}$ & $\mathbf{1 3 4 6}$ & $\mathbf{1 3 9 8}$ \\
\hline
\end{tabular}

Source : Rapports annuels de l'Alcan

En dépit de certaines fluctuations, la progression de la Société, au double point de vue de ses activités industrielles et commerciales, a donc été relativement régulière. C'est que le marché mondial de l'aluminium, métal aux mille usages, n'a pas cessé de se développer. En un quart de siècle, soit de 1946 à 1968, la consommation mondiale de ce métal a plus que triplé, passant de 2 300000 à 9250000 tonnes. Cette évolution favorable n'a fait que consolider les assises financières internationales de l'Alcan.

2.4.2 Le caractère de grande unité inter-territoriale de l'Alcan apparaît à travers la répartition géographique des principaux postes de son bilan. Le tableau analytique suivant a l'avantage de faire ressortir cet important aspect de la Société. 
Tableau 12

Alcan Aluminium Limitée et filiales

Bilan consolidé au 31 décembre 1971, (en millions de \$)

\begin{tabular}{|l|c|c|c|c|c|}
\hline \multicolumn{1}{|c|}{ Postes } & $\begin{array}{c}\text { Amérique } \\
\text { du Nord }\end{array}$ & $\begin{array}{c}\text { Amérique } \\
\text { du Sud } \\
\text { et Antilles }\end{array}$ & $\begin{array}{c}\text { Royaume-Uni } \\
\text { et Europe } \\
\text { continentale }\end{array}$ & Autres pays & Total \\
\hline ACTIF & 416 & 86 & 224 & 103 & 829 \\
\hline Disponible & 12 & 8 & 96 & 58 & 174 \\
\hline Investissements & 1532 & 284 & 359 & 237 & 2412 \\
\hline Immobilisations & $(837)$ & $(142)$ & $(127)$ & $(82)$ & $(1188)$ \\
\hline (Moins amortissements) & 12 & 59 & $(1)$ & -- & 70 \\
\hline Autres & 1135 & 295 & 551 & 316 & 2297 \\
\hline & 181 & 57 & 131 & 60 & 429 \\
\hline PASSIF & 510 & 37 & 137 & 56 & 740 \\
\hline À court terme & 129 & 2 & 3 & 8 & 142 \\
\hline À plus d'un an & 45 & 11 & 17 & 41 & 114 \\
\hline $\begin{array}{l}\text { Impôts/revenus par } \\
\text { participation }\end{array}$ & 214 & 188 & 263 & 151 & 816 \\
\hline $\begin{array}{l}\text { Intérêts minoritaires } \\
\text { filiales }\end{array}$ & 56 & -- & -- & -- & 56 \\
\hline $\begin{array}{l}\text { Actions privilégiées } \\
\text { Alcan }\end{array}$ & 921 & 107 & 288 & 165 & 1481 \\
\hline & & & & & \\
\hline $\begin{array}{l}\text { CAPITAL-ACTIONS } \\
\text { Ordinaires et bénéfices } \\
\text { non répartis }\end{array}$ & & & & & \\
\hline
\end{tabular}

Source : Alcan, Rapport annuel 1971.

Comme on le voit, la Société et ses filiales avaient une valeur d'actif de plus de deux milliards de dollars U.S, à la fin de 1971, après déduction des amortissements, répartis à travers tous les continents. Il importe de souligner cependant que le développement de la Compagnie n'a pas atténué son caractère d'origine, d'entreprise nord-américaine. L'Amérique du Nord retient plus de 60\% des immobilisations de la Société. Les 56 millions 
de dollars d'actions privilégiées Alcan sont détenues par des résidents de ce continent et sur les 32946549 actions ordinaires en cours de la Compagnie, plus de 85\% se trouvent entre les mains de Nord-Américains, soit exactement 55\% au Canada et 32,5\% aux États-Unis. Plus du tiers du personnel international de la Société travaille en Amérique du Nord, comme il apparaît au tableau ci-dessous.

Tableau 13

Répartition géographique du personnel Alcan, fin 1971

\begin{tabular}{|l|c|}
\hline Canada & 18300 \\
\hline États-Unis & 4500 \\
\hline Royaume-Uni & 9100 \\
\hline Antilles & 4500 \\
\hline Europe Continentale & 6900 \\
\hline Amérique Latine & 5900 \\
\hline Asie & 7300 \\
\hline Afrique & 1900 \\
\hline Océanie & 2200 \\
\hline Total & $\mathbf{6 0 6 0 0}$ \\
\hline
\end{tabular}

Source : Précis Alcan 1972.

Tous les développements qui précèdent montrent sans équivoque que l'Alcan est une grande unité inter-territoriale. On prendra connaissance dans les pages qui suivent de deux tableaux significatifs, dont le mérite est de synthétiser la situation globale de cette entreprise et son évolution récente. On peut d'ores et déjà prévoir que l'Alcan, en tant que société multinationale, est susceptible d'exercer des effets de domination sur l'économie du Saguenay-Lac-St-Jean. Cet aspect sera analysé dans la troisième partie de ce présent travail. Auparavant, on tentera de situer la Compagnie dans son contexte national canadien et régional saguenayen. 
Tableau 14

Alcan Aluminium Limitée

Rétrospective décennale

\begin{tabular}{|c|c|c|c|c|c|}
\hline & 1962 & 1965 & 1967 & 1969 & 1971 \\
\hline $\begin{array}{l}\text { Production totale d'aluminium } \\
\text { (milliers de tonnes) }\end{array}$ & 790 & 997 & 1399 & 1689 & 1865 \\
\hline - $\quad$ au Canada & 596 & 728 & 878 & 969 & 945 \\
\hline hors du Canada & 194 & 269 & 521 & 720 & 920 \\
\hline Ventes totales d'aluminium & 730 & 995 & 1104 & 1363 & 1398 \\
\hline - $\quad$ métal brut & 471 & 505 & 563 & 742 & 626 \\
\hline produits finis et semi-finis & 259 & 490 & 541 & 621 & 772 \\
\hline $\begin{array}{l}\text { Chiffre d'affaires } \\
\text { (millions de dollars US) }\end{array}$ & 521 & 838 & 934 & 1250 & 1449 \\
\hline - $\quad$ métal brut & 194 & 224 & 249 & 342 & 284 \\
\hline produits finis & 231 & 461 & 514 & 611 & 821 \\
\hline autres produits & 40 & 87 & 104 & 224 & 277 \\
\hline revenus d'exploitation et autres & 56 & 66 & 67 & 73 & 67 \\
\hline Actif total & 1378 & 1586 & 1823 & 2047 & 2297 \\
\hline - $\quad$ capitaux propres & 461 & 592 & 710 & 808 & 872 \\
\hline
\end{tabular}

Source : Extraits des rapports annuels de l'Alcan Aluminium Limitée, 1971. 
Tableau 15

Alcan Aluminium Limitée. Filiales et participations

\begin{tabular}{|c|c|}
\hline AMÉRIQUE DU NORD & AMÉRIQUE LATINE \\
\hline Canada & Argentine \\
\hline Aluminium du Canada Ltée & Camea S.A.I.C.*** \\
\hline Alcan Pipe Limired* & Brésil \\
\hline Produit Alcan Canada & Alcan Aluminio do Brasil S.A. \\
\hline Aluminium Goods Limired & Aluminio do Brasil Nordeste \\
\hline Canada Foils Limited & Aluminio Minas Gerais S.A. \\
\hline Forces Motrices du Saguenay Ltée & Mineraçao Rio do Norte S.A. \\
\hline Chemin de fer Alma et Jonquière & Colombie \\
\hline Compagnie de Chemin de Fer Roberval-Saguenay & Aluminio Alcan de Colombia* \\
\hline Maisons Alcan Limitée & Mexique \\
\hline Produits de bâtiment Alcan Ltée & Alcan Aluminio S.A.* \\
\hline Saguenay Shipping Limited & Uruguay \\
\hline Suprême Aluminium Industries Ltd*** & Alcan Aluminio del Uruguay* \\
\hline Transport d’Énergie du Saguenay & Venezuela \\
\hline États-Unis & Alcan de Venezuela \\
\hline Alcan Aluminium Corporation & AFRIQUE \\
\hline Alcan-LSI Hawaian Homes** & Ghana \\
\hline Fabral Corporation** & Ghana Aluminium Products Ltd \\
\hline Bermudes & Guinée \\
\hline Alcan Bermuda Limited & Halco (Mining) Inc*** \\
\hline Jamaïque & Nigeria \\
\hline Sprotons Jamaica Limited & Alcan Aluminium of Nigeria* \\
\hline Alcan Jamaica Limited & Flag Aluminium Products* \\
\hline Alcan products of Jamaica Ltd & République Sud-Africaine \\
\hline Trinidad & Alcan Aluminium of South Africa Limited* \\
\hline Chaguaramas Terminals Limited & Republic Aluminium Company (Pty) Limited \\
\hline \multicolumn{2}{|l|}{ Sprotons Trinidad Limited } \\
\hline EUROPE & EUROPE (suite) \\
\hline Allemagne & Norvège \\
\hline Alcan Aluminiumwerkw GmbH & A/S Ardal og Sunndal Werk** \\
\hline $\begin{array}{l}\text { Alcan Folienwerke GmbH \& Co. Kommanditgesell- } \\
\text { schaft* }\end{array}$ & A/S Nordisk Aluminiumindustri** \\
\hline
\end{tabular}




\begin{tabular}{|c|c|}
\hline Aluminium Norf GmbH** & DNN Aluminium A/S** \\
\hline Belgique & République d'Irlande \\
\hline Alcan Aluminium Raeren S.A. & Unidare Limited $* * *$ \\
\hline Danemark & Royaume-Uni \\
\hline Aluminord A/S*** & Alcan Booth industries Ltd* \\
\hline Espagne & Alcan Castings and Fortings Limited* \\
\hline Empresa Nacional del Aluminio*** & Alcan Design Products Ltd* \\
\hline France & Alcan Ekco Limited*** \\
\hline Aluminium Alcan de France & Alcan Enfield Alloys Ltd \\
\hline Alcan-Schwartz, Filage et Oxydation*** & Alcan Foils Limited * \\
\hline S.A. des Bauxites et Alumines de Provence & Alcan Polyfoil Limited * \\
\hline $\begin{array}{l}\text { Société Industrielle de Transformation et de } \\
\text { Construction*** }\end{array}$ & Alcan (U.K.) Limited \\
\hline Italie & Alcan Wire Limited* \\
\hline Alcan Aluminio Italanio S.p.A. & P.J. Bailey Limited* \\
\hline Alcan Angeletti \& Ciucani Aluminio* & Thomas Bennett Ltd*** \\
\hline PACIFIQUE SUD & Bonallack \& Sons Ltd* \\
\hline Australie & Freight Deveolpment Co. Ltd* \\
\hline Alcan Austratlia Limited* & Hunter Aluminium Co. Ltd $* * *$ \\
\hline AlcanQueensland Pty Limited & $\begin{array}{l}\text { Johnson and Bloy Aluminium Pigments } \\
\text { Limited *** }\end{array}$ \\
\hline Kawneer Company Pty Limited* & E.C. Payter \& Co. Ltd * \\
\hline Queensland Aluminia Limited*** & Saguenay Shipping (U.K.) Ltd.* \\
\hline Wm. Breit \& Co. Pty Ltd* & Tenon Contracts Limited * \\
\hline Nouvelle-Zélande & Suède \\
\hline $\begin{array}{l}\text { Alcan New Zealand Ltd* et Aluminium Conductors } \\
\text { Limited*** }\end{array}$ & Granges Essem $\mathrm{AB} * * *$ \\
\hline ASIE & Suisse \\
\hline Inde & Aluminiumwerke A.G. Rorschach \\
\hline Indian Aluminium Co. Ltd* & ASIE (suite) \\
\hline Japon & Malaisie \\
\hline Toyo Aluminium Co. Ltd** & Alcan Malaysia Berhad * \\
\hline Nippon Light Metal Co. Ltd ** & Southeast Asia bauxite Ltd* \\
\hline Thaïlande & Johore Mining and Stevedoring Co. Ltd * \\
\hline Alcan Thai Company Ltd** & \\
\hline
\end{tabular}

Filiale à $100 \%$; * Filiale à moins de $100 \%$; ** Filiale à 50\% ; *** Filiale à moins de $50 \%$. Source : Précis Alcan 1972. 


\section{Section 2 : Alcan, entreprise nationale}

$\underline{\text { Retour à la table des matières }}$

Il a été établi précédemment comment l'Alcan a pris naissance au Canada. L'Aluminium du Canada Limitée, dont le siège social est à Montréal, possède un peu plus de la moitié des immobilisations du holding Alcan Aluminium Limitée. Ses immobilisations, à la fin de 1971, étaient estimées à 770 millions de dollars, soit $63 \%$ de l'ensemble des immobilisations d'Alcan Aluminium Limitée à travers le monde. La principale filiale, responsable de la commercialisation des produits fabriqués par la Compagnie, « Produits Alcan Canada Limitée », a son siège social à Toronto. Vers la fin de 1971, les effectifs engagés par la Société à travers ses entreprises canadiennes s'élevaient à environ 18000 personnes, soit 30\% de l'ensemble du personnel Alcan. Le caractère essentiellement canadien de la Compagnie ressort également de la nationalité de son capital-actions. Les actions privilégiées, à la valeur nominale de près de 55 millions de dollars, sont presque entièrement aux mains du public canadien. Au 31 décembre 1972, plus de 55 \% des porteurs des actions ordinaires de la Société avaient leur domicile au Canada. Le poids de l'Alcan dans l'économie du pays est donc déterminant à plus d'un titre. Certains auteurs notent même «un certain parallélisme entre la croissance du Canada et l'expansion de l'industrie de l'aluminium qui se range parmi les entreprises les plus importantes du pays » 88.

Deux aspects seront pris en considération dans les paragraphes suivants : la diversité des activités industrielles de l'Alcan au Canada et la forte position subséquente de ce pays sur le marché mondial de l'aluminium.

88 Mc Guire, J. Sarrazin, op. cit., p.5. 


\subsection{Le contexte canadien de l'Alcan}

$\underline{\text { Retour à la table des matières }}$

L'Aluminium du Canada, Limitée est responsable d'environ 80\% de la production canadienne d'aluminium. L'activité industrielle de la Société est très diversifiée et s'étend à travers tout le pays, en particulier, dans les provinces de Québec, de l'Ontario et de la Colombie-Britannique.

En 1971, sur l'ensemble de la production du métal de première fusion du Groupe Alcan, plus de la moitié, soit 945 milliers de tonnes, a été produite par des entreprises installées au Canada. Quoique ce pays soit l'un de ceux où la consommation d'aluminium soit la plus élevée par tête et par an (25 livres), le marché canadien n'absorbe que 15\% environ de l'aluminium produit par l'Alcan, 85 \% devant être écoulé en dehors du Canada.

De fait, reconnaissent volontiers les responsables de la Compagnie, «seule l'exportation a permis à la Société d'atteindre le rang qu'elle occupe au Canada et sur le marché mondial de l'aluminium » 89.

Les conditions particulièrement favorables rencontrées dans l'ensemble du pays, en ce qui concerne notamment la production de l'énergie hydroélectrique, ont été mises à profit pour rendre l'industrie canadienne d'aluminium l'une des plus compétitives du monde.

La politique économique, résolument libérale, des gouvernements successifs de ce pays a également favorisé les plans d'expansion de l'Alcan, dont « l'organisation industrielle et commerciale repose sur la conviction que les échanges internationaux sont bienfaisants et nécessaires et que chaque pays devrait employer ses ressources à la production des biens dans laquelle il excelle » 90. Mais, l'Alcan ne s'est pas cantonnée dans la seule production de l'aluminium brut, ainsi qu'on peut l'observer dans le tableau qui suit.

89 Alcan, Précis Alcan, 1969, p. 12.

90 Alcan, idem, p. 12. 
Tableau 16

Alcan : activités industrielles à travers le Canada, 1971

\begin{tabular}{|c|c|}
\hline Entreprises & Activités ou produits \\
\hline $\begin{array}{ll}\text { Usines d'électrolyse } \\
\text { - } & \text { Arvida (Québec) } \\
\text { - } & \text { Beauharnois (Québec) } \\
\text { - } & \text { Isle-Maligne (Québec) } \\
\text { - } & \text { Shawinigan (Québec) } \\
\text { - } & \text { Kitimat (Colombie-Britanique) }\end{array}$ & Aluminium de première transformation \\
\hline $\begin{array}{l}\text { Usines de produits chimiques } \\
\text { - } \quad \text { Arvida (Québec) } \\
\text { - } \quad \text { Shawinigan } \\
\text { - } \quad \text { Ottawa (Ontario) }\end{array}$ & $\begin{array}{l}\text { Alumine, sulfate d'aluminium, fluorure, cryolite chlore } \\
\text { Sulfate d'aluminium } \\
\text { Sulfate d'aluminium }\end{array}$ \\
\hline $\begin{array}{cl}\text { Usines de transformation } \\
\text { - } & \text { Arvida (Québec) } \\
\text { - } & \text { Aurora (Ontario) } \\
\text { - } & \text { Bracebridge (Ontario) } \\
\text { - } & \text { Rexdale (Ontario) } \\
\text { - } & \text { Laval (Québec) } \\
\text { - } & \text { Richmond (Vancouver C.-B.) } \\
\text { - } & \text { Shawinigan (Québec) } \\
\text { - } & \text { Stephenville (Terre-Neuve) }\end{array}$ & $\begin{array}{l}\text { Grenailles d'aluminium, alpâte, relaminage } \\
\text { Profilés, anodisation, peinture } \\
\text { Feuille mince d'aluminium } \\
\text { Profilés, tubes d'aluminium, tôles en aluminium } \\
\text { Articles en feuille mince } \\
\text { Profilés, anodisation, peinture } \\
\text { Fils-machine, fils et câbles } \\
\text { Câbles, fils et articles pour conducteurs } \\
\text { Câble nu }\end{array}$ \\
\hline $\begin{array}{l}\text { Filiales ou Sociétés affiliées } \\
\text { - Alcan building products Ltd } \\
\text { - Maisons Alcan Limitée } \\
\text { - } \text { Maison Universelle } \\
\text { - Alcan Pipe Limited } \\
\text { - Aluminium Goods Limited } \\
\text { - Canada Foils Limited } \\
\text { - Supreme Aluminium Industries Ltd }\end{array}$ & $\begin{array}{l}\text { Produits du bâtiment } \\
\text { Construction de maisons } \\
\text { Fabrication de maisons montées en usines } \\
\text { Tuyaux et conduites d'irrigation } \\
\text { Produits en aluminium, casserollerie } \\
\text { Aluminium souple et autres produits d'emballage } \\
\text { Tôle, ustensiles de cuisine }\end{array}$ \\
\hline
\end{tabular}

Source : Alcan : Précis Alcan, 1969, p. 12. Précis Alcan 1971. 
L'inventaire qui précède donne une idée valable des principaux domaines d'activité industrielle de la Société au Canada. Il n'est pas cependant pas limitatif, puisque l'Alcan exploite ellemême des centrales hydroélectriques et pourvoit à la majeure partie de ses besoins dans le domaine des transports. Ce double aspect des activités de la Compagnie sera analysé de façon plus particulière à la troisième section du présent chapitre.

\subsection{L'expansion de l'Alcan et la position du Canada sur le marché mondial de l'aluminium}

\section{$\underline{\text { Retour à la table des matières }}$}

Le rôle majeur que joue l'aluminium dans l'industrie mondiale n'est plus à démontrer aujourd'hui. Les principaux domaines dans lesquels on en fait usage sont la construction, les transports, l'industrie de matériel électrique et les emballages. De plus, l'aluminium est essentiel à l'aviation, ce qui en fait un métal stratégique de la plus haute importance. Aussi n'est-il pas étonnant que la plupart des pays industrialisés aient cherché à développer sur leurs territoires l'industrie du «métal de la victoire ». Fruit d'une politique délibérée ou résultat du «laisser-faire », le Canada est devenu, grâce à l'Alcan, l'un des plus grands producteurs d'aluminium du monde. Il n'est peut-être pas inutile de suivre le chemin parcouru par ce pays dans ce domaine 91. Presque nulle au début du XXe siècle, la production canadienne d'aluminium a connu un rythme d'accroissement très rapide, stimulée en cela par les deux dernières guerres mondiales. Mais, comme on l'a déjà souligné, la production canadienne se confond presque avec celle de l'Alcan. Aujourd'hui, avec 12,5\% environ de la production du monde non-communiste 92, le Canada occupe le deuxième rang parmi les pays producteurs, après les États-Unis. Seule l'exportation, dont l'évolution a été sensiblement parallèle à celle de la production, a permis ce développement dont la courbe est tracée à la page suivante.

91 Voir le tableau 17.

92 En 1971, l’URSS a produit près de 2 millions de tonnes. 
Tableau 17

Production d'aluminium Monde non-communiste, Canada, (en tonnes)

\begin{tabular}{|c|c|c|}
\hline $\begin{array}{c}\text { Années } \\
\text { caractéristiques }\end{array}$ & Monde non-communiste & Canada \\
\hline 1900 & 7000 & ---- \\
\hline 1910 & 44000 & 4400 \\
\hline 1920 & 127000 & 12000 \\
\hline 1930 & 265000 & 30000 \\
\hline 1939 & 688000 & 75000 \\
\hline 1950 & 1508000 & 360000 \\
\hline 1959 & 3390000 & 543700 \\
\hline 1968 & 7250000 & 873000 \\
\hline 1971 & 8920000 & 1105000 \\
\hline
\end{tabular}

Sources : R. Gadeau, L'aluminium, Éd. A. Colin, 1958, p. 43.

Annuaire statistique des Nations Unies.

Year Book 1971 of American Bureau of Metal Statistics 


\section{Graphique 3}

Production d'aluminium au Canada 1910-1968

Retour à la table des matières

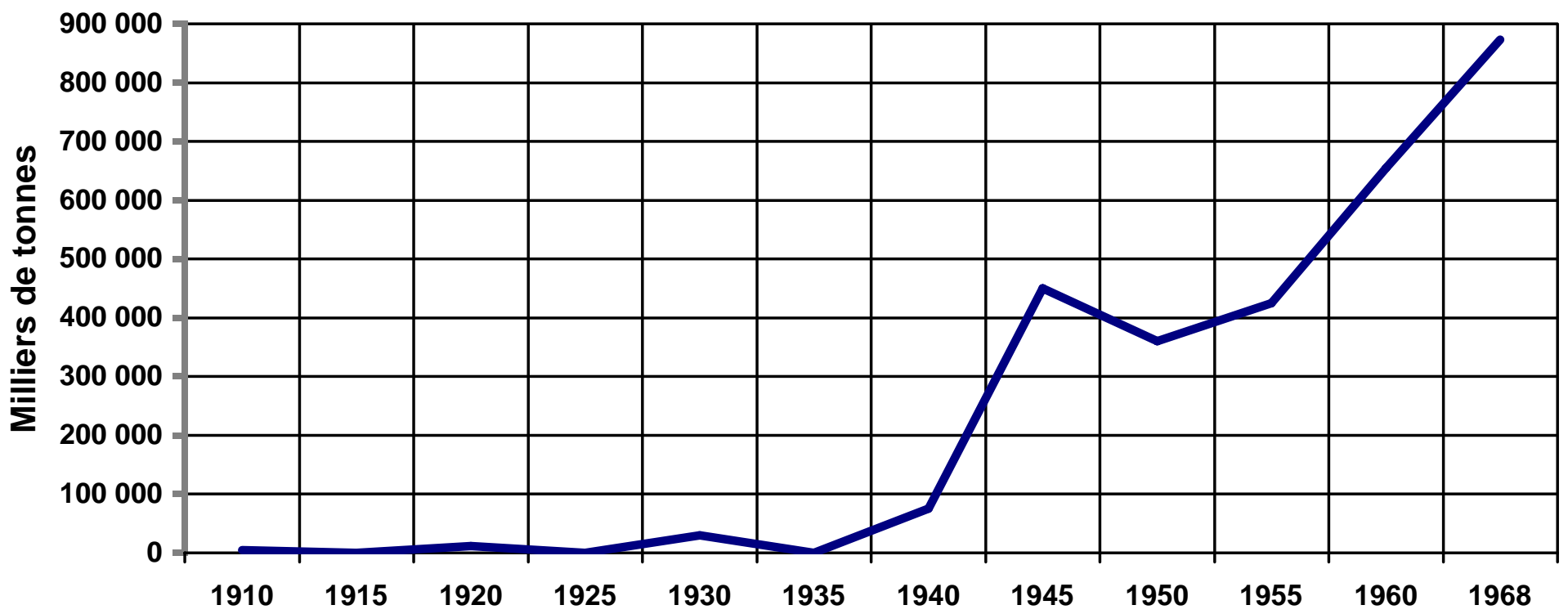




\section{Section 3 : Alcan, entreprise régionale}

$\underline{\text { Retour à la table des matières }}$

Par la construction de l'usine d'aluminium d'Arvida, l'Alcan, dans le deuxième quart du siècle, avait fait son entrée sur la scène de l'économie du Saguenay-Lac-St-Jean. Le développement de cette entreprise n'a pas manqué de modifier la morphologie socioéconomique de la région. Cette évolution, effectuée sans doute en fonction de la stratégie propre de la Société, s'est opérée dans trois directions majeures qui constitueront les axes de l'analyse qui va suivre.

\subsection{Le développement du réseau hydroélectrique}

En 1926, au moment de la mise en marche, l'usine d'aluminium d'Arvida était ravitaillée en énergie électrique par la centrale d'Isle-Maligne, dont la capacité, relativement importante pour l'époque, s'élevait à 402000 kW. Quoique sous le contrôle de l'Alcan, la centrale d'Isle-Maligne constituait la source d'approvisionnement de plusieurs autres entreprises de la région. L'énergie disponible était cependant insuffisante pour répondre aux exigences des plans d'expansion à long terme de la Société. C'est donc à la solution de ce problème vital que l'Alcan consacrera ses premiers efforts.

Dès 1927, elle procéda à l'ouverture des chantiers de construction d'une nouvelle centrale sur la Chute-à-Caron, située à 21 milles en aval de l'Isle-Maligne. Achevée quatre ans plus tard, cette nouvelle centrale pouvait fournir jusquà 224000 kW. Mais l'accroissement de la demande mondiale d'aluminium entraîné par l'éclatement de la seconde guerre mondiale constitua le véritable point de départ d'un va programme qui allait faire du Saguenay_Lac-St-Jean le principal centre de l'électrométallurgie du Québec. 
En 1941, pour augmenter la capacité de production de son usine d'Arvida, l'Alcan entreprit l'aménagement de la Rivière Shipshaw. Le résultat de ces travaux fut la centrale du même nom, dont la capacité ne s'élevait pas moins à 895000 kWh.

Presque à la même époque, dans le but de régulariser le débit de la Rivière Péribonka, la Société fit construire deux immenses barrages: le premier, au lac Manouane et le second sur les Passes Dangereuses. Additionnées, la capacité de ces deux réservoirs artificiels atteignaient 270 milliards de pieds cubes, potentialités qui seront utilisées plus tard. Mais, déjà vers 1947, la région avait enregistré de sensibles progrès, ainsi qu'il apparaît au tableau suivant.

\section{Tableau 18}

\section{Le développement hydroélectrique du Saguenay-Lac-St-Jean}

(en chevaux-vapeur)

\begin{tabular}{|l|r|l|r|}
\hline Rivière Ha ! Ha ! & 2300 & Shipshaw * & 1200000 \\
\hline Rivière à Mars & 1300 & Murdock & 10800 \\
\hline Rivière Chicoutimi & 8500 & Chute aux Galets & 17600 \\
\hline Pont-Arnaud & 7200 & Isle-Maligne * & 540000 \\
\hline Chute-Garneau & 3500 & Belle-Rivière & 1000 \\
\hline Jonquière & 2100 & Desbiens & 1400 \\
\hline Kénogami & 6000 & Val-Jalbert & 1000 \\
\hline St-Félicien & 230 & Chute-à-Caron * & 280000 \\
\hline St-Prime & 100 & & \\
\hline \multicolumn{2}{|c|}{ Total : } & $\mathbf{2 0 8 2 ~ 8 3 0}$ \\
\hline
\end{tabular}

* Centrale appartenant à l'Alcan.

Source : Saguenay Industrial Development Corporation, cité par B. Brouillette et P. Dagenais : op. cit., p. 672.

Vers 1947, sur le total des 2082830 chevaux-vapeur représentant la capacité globale des centrales hydroélectriques de la région, l'Alcan, à elle seule, fournissait plus de deux millions, soit 96\% environ. Or, à cette époque, la production énergétique, sous forme d'électricité, du Saguenay_Lac-St-Jean représentait près de 50\% de celle de toute la Province du Québec (5 500 000 ch.-vap.) et près du quart de celle du Canada dans son ensemble

(9 300000 ch.-vap.). 
C'est dire que le Saguenay_Lac-St-Jean venait en première ligne parmi les diverses régions canadiennes aptes à l'industrialisation. Et ce développement se poursuivit encore : en 1968, les centrales appartenant à l'Alcan fournissaient, à elles seules, plus de 3,5 millions de chevaux-vapeur, soit exactement $2687000 \mathrm{kWh}$, répartis en six grandes centrales 93.

Tableau 19

Centrales hydroélectriques de la région appartenant à l'Alcan

$\underline{\text { Retour à la table des matières }}$

\begin{tabular}{|c|c|c|c|}
\hline Localisation & Date de mise en marche & Puissance en KW & Rivières \\
\hline Isle-Maligne & 1926 & 402000 & Saguenay \\
\hline Chute-à-Caron & 1931 & 224000 & Saguenay \\
\hline Shipshaw & 1943 & 896000 & Saguenay \\
\hline Chute-du-Diable & 1953 & 205000 & Péribonka \\
\hline Chute-à-la-Savanne & 1953 & 210000 & Péribonka \\
\hline Chute-des-Passes & 1960 & 750000 & Péribonka \\
\hline \multicolumn{2}{|l|}{ Total } & 2687000 & \\
\hline
\end{tabular}

Source : Précis Alcan, 1971.

93 Voir la carte 3. 
Carte 3.

les centrale hydroélectriques du Saguenay_Lac-St-Jean 1968

$\underline{\text { Retour à la table des matières }}$

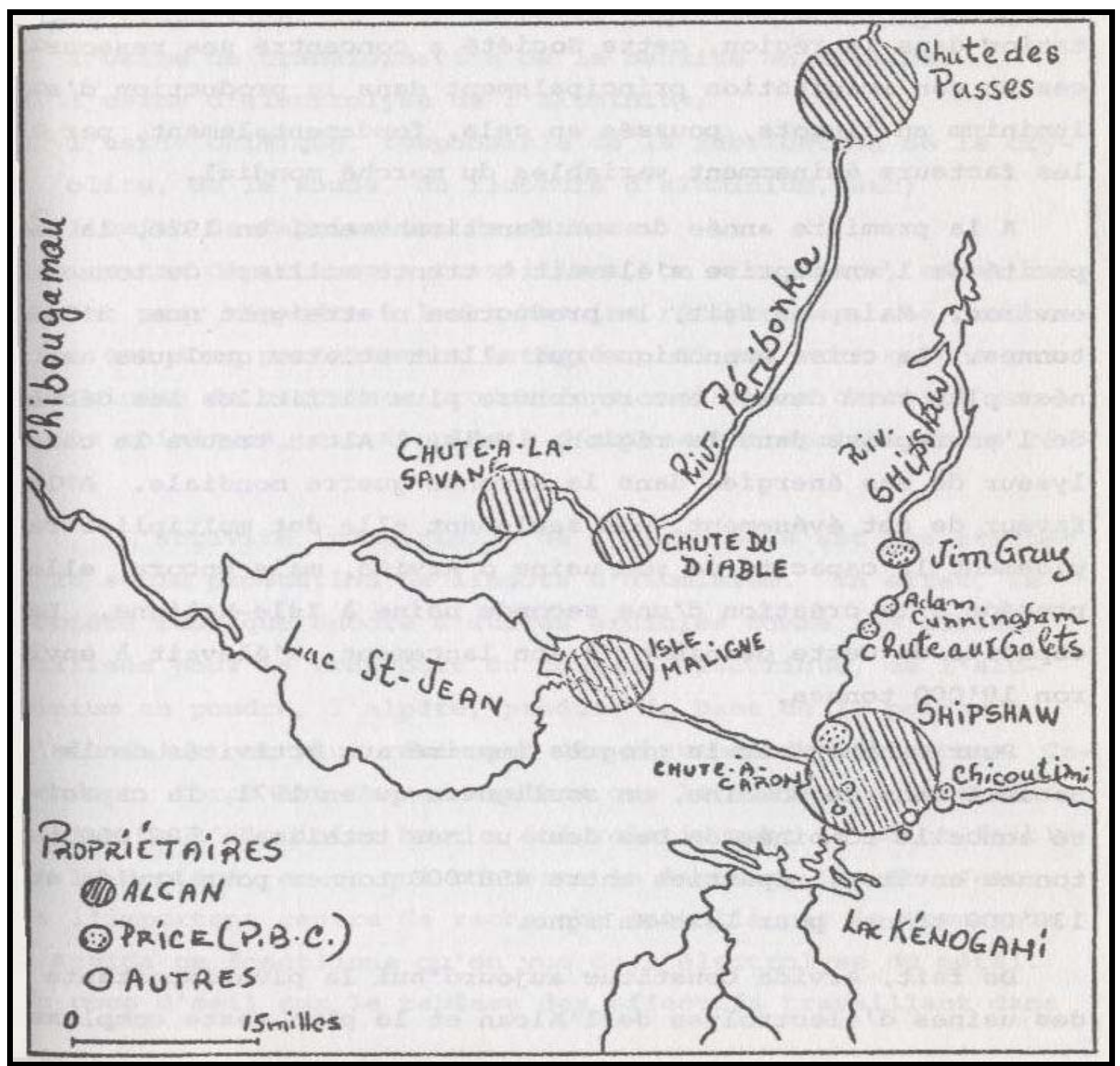




\subsection{L’industrie régionale d’aluminium}

$\underline{\text { Retour à la table des matières }}$

La considérable expansion de la production hydroélectrique du Saguenay-Lac-St-Jean, retracée dans les pages précédentes, ne se comprend que replacée dans le contexte des plans de développement à long terme de l'Alcan. Or, depuis son implantation dans la région, cette Société a concentré ses ressources et son imagination principalement dans la production d'aluminium en lingots, poussée en cela, fondamentalement, par les facteurs éminemment variables du marché mondial.

À la première année de son fonctionnement, en 1926, la capacité de l'entreprise s'élevait à trente milliers de tonnes environ. Mais, en fait, la production n'atteignit que 3949 tonnes. La crise économique qui allait éclater quelques années plus tard devait encore rendre plus difficiles les débuts de l'entreprise dans la région. Mais, l'Alcan trouva le catalyseur de ses énergies dans la seconde guerre mondiale. À la faveur de cet événement, non seulement elle dut multiplier rapidement la capacité de son usine d'Arvida, mais encore elle procéda à la création d'une seconde usine à IsleMaligne. La capacité de cette dernière, à son lancement, s'élevait à environ 18000 tonnes. Pour synthétiser le progrès imprimé aux activités de la Société dans ce domaine, on soulignera qu'en 1971, la capacité annuelle combinée de ces deux usines totalisait 588000 tonnes environ, réparties entre 458000 tonnes pour Arvida et 130000 tonnes pour Isle-Maligne. De fait, Arvida constitue aujourd'hui la plus importante des usines d'électrolyse de l'Alcan et le plus vaste complexe d'aluminium du monde. Quelques données préciseront encore mieux cette observation. L'usine s'étend sur une superficie d'environ trois kilomètres carrés. Ses différentes sections sont reliées entre

elles par des voies ferrées dont la longueur totale dépasse quarante kilomètres. Quatre départements travaillent au sein de cette gigantesque entreprise :

- l'usine de transformation de la bauxite en alumine ;

- l'usine d'électrolyse de l'aluminium ;

- l'usine chimique, responsable de la fabrication de la cryolite, de la soude, du fluorure d'aluminium, etc. ; 
- l'usine de fabrication de carbone.

Ces quatre départements travaillent, on s'en doute, essentiellement pour répondre à leurs besoins réciproques. La production est donc parfaitement intégrée. Il est vrai qu'une partie minime de certaines productions, notamment certains produits chimiques, est écoulée à d'autres entreprises de la région.

L'activité industrielle de l'Alcan ne s'est pas arrêtée à la seule production de lingots d'aluminium. En effet, la Société fabrique encore d'autres articles comme les câbles, utilisés pour le transport du courant électrique, de l'aluminium en poudre, l'alpâte, produit de base de la peinture d'aluminium, et plus récemment des ébauches de relaminage. Cependant, force est de reconnaître que le secteur de transformation de l'aluminium brut a été relativement le point faible de la Société, en ce qui concerne le Saguenay_Lac-St- Jean . Et l'important centre de recherche installé par la Compagnie à Arvida ne fonctionne qu'en vue de l'électrolyse du métal. Un coup d'œil sur le tableau des effectifs travaillant dans les entreprises régionales de l'Alcan confirme que la production d'aluminium brut reste la principale préoccupation de la Société.

Tableau 20

Alcan : main-d'œuvre et salaires, région 1967

\begin{tabular}{|l|r|r|}
\hline & Unités & $\mathbf{1 0 0 0}$ \\
\hline Arvida & 6000 & 50000 \\
\hline Isle-Maligne & 1000 & 7500 \\
\hline Saguenay Terminals Ltd & 700 & \\
\hline Chemin de fer Roberval Saguenay & & \\
\hline Chemin de fer Alma-Jonquière & 200 & \\
\hline Division hydroélectrique & 300 & 19900 \\
\hline Forces Motrices du Saguenay & 150 & \\
\hline Aluminium Laboratories & 150 & \\
\hline Services divers & 500 & \\
\hline & 9000 & 77400 \\
\hline
\end{tabular}

Source : Renseignements fournis par l'Alcan, Service des relations extérieures. 
Ainsi près de 80\% de la main-d'œuvre de l'Alcan, au Saguenay-Lac-St-Jean, travaillaient dans la production de l'aluminium de première fusion, dans les deux usines d'Arvida et d'Isle-Maligne. Les 70 personnes embauchées pour la mise en marche, en 1971, de l'usine de relaminage d'Arvida n'ont pas modifié cette donnée de manière significative. En fait, il semble que le personnel de l'Alcan, dans la région, se soit plus ou moins stabilisé autour de 9000 employés.

Il n'en a pas toujours été ainsi, si on se réfère aux 1650 employés de l'usine d'Arvida en 1927. Du reste, il faut bien admettre que la main-d'œuvre au service de l'Alcan a toujours été soumise aux vicissitudes de la conjoncture mondiale. Et pour cause, puisque, comme il a été déjà souligné, les activités de la Société sont étroitement liées à ses possibilités d'exportation. Ainsi, on vit le personnel d'Arvida tomber de 1650 unités qu'il était en 1927 à 400 seulement en 1932, au plus fort de la grande dépression et remonter en 1943 à 11800 (volume du personnel de toutes les entreprises régionales de l'Alcan). Ce chiffre d'ailleurs ne sera jamais plus atteint.

Toutes ces considérations traduisent, à coup sur, l'importante évolution qui a été enregistrée par les activités industrielles de l'Alcan au Saguenay_Lac-St-Jean. D'ores et déjà, on peut souligner, que liée à la société, pour le meilleur et pour le pire, l'économie de la région n'a pas manqué d'être marquée par l'existence de cette dynamique entreprise. Encore convient-il auparavant de voir le dernier domaine dans lequel la Compagnie se devait de porter ses efforts.

\subsection{L'Alcan et le problème des transports régionaux}

$\underline{\text { Retour à la table des matières }}$

C'est particulièrement dans le domaine des transports maritimes que l'action de l'Alcan s'est exercée dans la région. Cela se comprend, si l'on se rappelle que les matières premières de l'industrie saguenayenne d'aluminium, notamment la bauxite, proviennent en totalité de l'extérieur du Canada. À ce point de vue, la Société était merveilleusement servie par la voie d'eau du 
Saguenay. L'aménagement de cette rivière, la modernisation de ses ports, autant de tâches qui s'avéraient indispensables à la bonne marche et au développement des activités industrielles de la Compagnie.

Aujourd'hui, souligne P.-Y. Pépin, le Saguenay-Lac-St-Jean compte «un seul pivot maritime », Port-Alfred, qui aurait été voué à un « destin mineur » sans la dynamique présence des entreprises de l'Alcan. En effet, avant l'implantation de la compagnie, Port-Alfred desservait principalement les quelques fabriques de papier de la région. Or, on sait que cette industrie ne prit véritablement son élan que postérieurement à l'arrivée de l'Alcan dans le Saguenay et à l'ère industrielle que cet événement devait inaugurer. Dès 1925, les installations de Port-Alfred passèrent aux mains de l'Alcan qui, par l'intermédiaire de sa filiale, le « Saguenay Terminals Limited », devait en faire, consécutivement à des aménagements appropriés, l'un des ports les plus modernes du Canada. Il en résulta naturellement une intensification du trafic global.

Avant la seconde guerre mondiale, le trafic global du port oscillait autour de 600000 tonnes annuellement. En 1965, il atteignait près de quatre millions de tonnes, soit six fois plus que celui de Chicoutimi, le deuxième port régional. Incontestablement, cette rapide expansion doit être mise, en majeure partie, au crédit de l'Alcan, dont les importantes commandes et expéditions forment un pourcentage élevé de ce trafic, comme on peut s'en rendre compte au tableau suivant. 
Tableau 21

Port-Alfred : trafic global et bauxite, 1952-1965

$\underline{\text { Retour à la table des matières }}$

\begin{tabular}{|l|c|c|c|}
\hline \multirow{2}{*}{ Années } & Trafic total & \multicolumn{2}{|c|}{ Bauxite } \\
& $\mathbf{1} \mathbf{0 0 0}$ tonnes & $\mathbf{1 0 0 0}$ tonnes \% du total \\
\hline $\mathbf{1 9 5 2}$ & 3699 & 2294 & 62,0 \\
\hline $\mathbf{1 9 5 4}$ & 4058 & 2638 & 64,9 \\
\hline $\mathbf{1 9 5 6}$ & 3534 & 2130 & 60,2 \\
\hline $\mathbf{1 9 5 8}$ & 3016 & 1874 & 62,1 \\
\hline $\mathbf{1 9 6 0}$ & 3632 & 2278 & 62,7 \\
\hline $\mathbf{1 9 6 2}$ & 2727 & 1504 & 55,1 \\
\hline $\mathbf{1 9 6 5}$ & 3624 & 1850 & 51,0 \\
\hline Moyenne de la période & $\mathbf{3} 386$ & $\mathbf{2 0 3 6}$ & $\mathbf{5 9 , 7}$ \\
\hline
\end{tabular}

Source : Shipping Report, cité par J. Harvey, « L’agglomération portuaire Port-AlfredBagotville » dans Cahiers de Géographie du Québec, no 22, avril 1967, p. 33.

La part relativement énorme de la bauxite dans le trafic global, $60 \%$ en moyenne sur l'ensemble de la période considérée, traduit sans équivoque la dépendance de Port-Alfred à l'égard de l'industrie régionale d'aluminium. Ce phénomène est encore certainement plus important, si on tient compte des autres matières premières nécessaires à cette industrie et de la sortie des lingots d'aluminium produits par la Société. Il est toutefois bon de souligner que Port-Alfred est un entrepôt, en ce sens qu'il reçoit beaucoup plus qu'il n'expédie. À cet effet, le tableau suivant est très significatif. Néanmoins, la spécialisation du port et sa dépendance à l'égard de l'Alcan ne font aucun doute même si on a pu remarquer une atténuation progressive mais légère de ce double phénomène. 
Tableau 22

Port-Alfred, trafic 1965 (en tonnes)

$\underline{\text { Retour à la table des matières }}$

\begin{tabular}{|c|c|c|c|}
\hline \multicolumn{4}{|c|}{ Cargaisons océaniques } \\
\hline Produits & Sorties & Entrées & Total \\
\hline Billes et billots & 653 & 45 & 698 \\
\hline Bauxite et alumine & ---- & 2004621 & 2004621 \\
\hline Bitumeux & ---- & 113922 & 113922 \\
\hline Bois d'œuvre et en grumes & 8247 & -- -- & 8247 \\
\hline Papier journal & 286706 & -- -- & 286706 \\
\hline Cartons & 4676 & -- -- & 4676 \\
\hline Produits chimiques & -- -- & 24669 & 24669 \\
\hline Combustibles & ---- & 235504 & 235504 \\
\hline Coke & ---- & 164420 & 164420 \\
\hline Lingots d'aluminium & 125569 & -- -- & 125569 \\
\hline Marchandises érales & 4493 & 1200 & 5693 \\
\hline Autres & 25886 & 1982 & 27868 \\
\hline Total & 456230 & 2557156 & 3013286 \\
\hline \multicolumn{4}{|c|}{ Cargaisons côtières } \\
\hline Bois de pulpe & -- -- & 432584 & 432584 \\
\hline Minerai de fer et concentré & ---- & 1192 & 1192 \\
\hline Fluorine & --- & 111874 & 111874 \\
\hline Sel & -- -- & 61660 & 61600 \\
\hline Marchandises générales & 1542 & -- -- & 1542 \\
\hline Total & 1542 & 609192 & 610954 \\
\hline
\end{tabular}

Source : Saguenay Terminals Limited, cité par J. Harvey, op. cit., pp. 32-33.

L'examen des données concernant les produits qui ont transité par Port-Alfred, en 1965, confirme bien que ce port est une création purement commerciale de l'Alcan et, en second lieu, de l'industrie du papier. À côté de ces faits marquants, soulignés en ce qui concerne le domaine des transports maritimes, il faudrait encore ajouter que l'Alcan a participé à la mise en 
place des chemins de fer régionaux. On peut revoir ce qui a été déjà dit précédemment sur cet aspect de la question.

À la vérité, si les analyses qui précèdent font déjà pressentir le rôle éminent joué par l'Alcan au Saguenay_Lac-St-Jean, en particulier, et au Canada, en général, il n'en demeure pas moins que cette Société est fondamentalement une grande unité inter-territoriale. En tant que telle, l'Alcan bénéficie d'une autonomie certaine à l'égard du marché national canadien, et davantage encore face aux territoires particuliers d'implantation, comme le Saguenay-Lac- St-Jean. Quoiqu'il n'y ait pas incompatibilité de principe entre la stratégie des grandes firmes multinationales et la croissance plus ou moins équilibrée de leurs régions d'accueil, on ne peut s'empêcher d'être influencé au départ par certains préjugés en la matière. De manière plus explicite, une certaine littérature s'est développée qui laisse entendre que la firme multinationale, dominante par son poids économique et généralement étrangère à la région d'accueil, exploite cette dernière au double sens de ce terme. L'examen de l'économie du Saguenay_Lac St-Jean et des principales étapes de son évolution s'avère donc nécessaire. 
Deuxième partie :

L’Alcan et l'évolution de l'économie du S.-L.-S-.J.

Chapitre V

\section{Le repérage des indicateurs de la croissance économique du Saguenay_Lac-St-Jean}

$\underline{\text { Retour à la table des matières }}$

Dans la première partie de cette étude, on a souligné les traits caractéristiques de l'économie saguenayenne d'avant la venue de l'Alcan dans la région. Il importe, dans le présent chapitre, de prendre la mesure de l'économie du Saguenay_Lac-St-Jean à l'heure actuelle et d'analyser brièvement les phases principales de son évolution, en essayant de ponctuer les efforts effectués et les progrès réalisés par rapport à 1925, année de démarrage des activités de l'Alcan dans la région.

S'il est vrai que la croissance économique est fondamentalement « the growth of the output per head of population », comme le soutient Lewis 94, elle n'en demeure pas moins un phénomène complexe, mettant en jeu des facteurs multiples et de nature diverse. De toute manière, ce ne sera pas le lieu ici d'entreprendre une discussion sur le phénomène de la croissance économique. Tout simplement, on tentera de voir dans quelle mesure l'évolution de l'économie saguenayenne peut être identifiée à «une augmentation prolongée de la dimension de ses variables caractéristiques, accompagnée de changements de structure et éventuellement de progrès dans

94 W.A. Lewis, The Theory of Economic Growth, Homewood, 1955, p. 10. 
l'efficacité de l'effort et le bien-être » 95. Le présent chapitre comportera deux sections. La première aura pour objet la production régionale et la seconde, le revenu et le niveau de vie dans la région.

\section{Section 1 : La production régionale}

$\underline{\text { Retour à la table des matières }}$

Le produit intérieur brut du Saguenay-Lac-St-Jean, en 1966, s'élevait à 540 millions de dollars, soit un accroissement absolu de plus de 300 millions, par rapport à 1951. Dans le même temps, le PIB pat tête avait enregistré une augmentation de 759 dollars, passant de 1153 dollars, en 1951, à 1 912 dollars, en 1966. Il est évident que le taux d'accroissement est encore plus important si l'on compare ces données avec celles des années 1925-26. À première vue donc, l'économie du Saguenay_Lac-St-Jean aurait eu une évolution favorable. Toutefois, comparée au reste de la Province du Québec, la croissance régionale a été sensiblement inférieure, ainsi qu'il apparaît cidessous 96.

L'examen du tableau révèle que la production du Saguenay-Lac-St-Jean a connu une croissance relativement soutenue. Certes, sur la base de l'indice 100 en 1951, le produit intérieur brut est passé à 238 en 1966, ce qui se traduit par un taux d'accroissement relativement élevé de 66\% durant la même période. Cependant, ces données sont trompeuses, puisque elles ne tiennent pas compte de la dépréciation de la monnaie.

D'autre part, la comparaison de la situation de la région avec les autres parties du territoire provincial ne laisse pas d'être inquiétante, puisqu'elle révèle un net recul du Saguenay-Lac-St-Jean. En effet, le taux moyen de croissance de l'ensemble du Québec a été de 92\% , présentant un

95 F. Perroux, « Matériaux pour une analyse de la croissance économique », Cahiers de l'ISEA, avril 1955, p. 18.

96 Voir le tableau 23. 
écart de 26 \% par rapport à celui de la région. La même observation pourrait être faite en ce qui concerne le trend d’évolution du produit par tête pour les quinze années considérées.

Tableau 23

PIB par tête, Québec et régions

(en dollars courants et indice $1951=100$ )

Retour à la table des matières

\begin{tabular}{|c|c|c|c|c|c|c|}
\hline Régions & & $51(\$)$ & 196 & $(\$)$ & $\begin{array}{c}\text { Régions } \\
\text { Québec }\end{array}$ & Taux de \\
\hline 1. Gaspésie-Bas du Fleuve & 552 & 100 & 1221 & 100 & 54,7 & 121 \\
\hline 2. Saguenay-Lac-St-Jean & 1152 & 100 & 1912 & 100 & 85,7 & 66 \\
\hline 3. Québec (région) & 924 & 100 & 1693 & 100 & 75,9 & 105 \\
\hline 4. Trois-Rivières & 1100 & 100 & 1852 & 100 & 83,0 & 68 \\
\hline 5. Cantons de l'Est & 1158 & 100 & 1981 & 100 & 88,8 & 71 \\
\hline 6. Montréal (région) & 1365 & 100 & 2607 & 100 & 116,8 & 91 \\
\hline 7. Outaouais & 974 & 100 & 1783 & 100 & 79,9 & 83 \\
\hline 8. Nord-Ouest & 1298 & 100 & 1552 & 100 & 69,5 & 20 \\
\hline 9. et 10. Côte-Nord et Nouveau-Québec & 971 & 100 & 2573 & 100 & 115,3 & 165 \\
\hline Province de Québec & 1160 & 100 & 2232 & 100 & 79,0 & 92 \\
\hline Province - Montréal & 953 & 100 & 1760 & 100 & 79,0 & 85 \\
\hline
\end{tabular}

Source: Saicans, A., Analyse des principales variables économiques du Saguenay-Lac-St-Jean , OPDQ, août 1969, ronéotypé. pp. 31-33.

On doit cependant souligner que l'exploitation des mines du Nouveau-Québec avec le boom économique qui en a résulté et la singulière expansion industrielle de la région montréalaise, traditionnellement plus riche, contribuent quelque peu à fausser la comparaison, par une certaine sous-estimation des performances réalisées par les autres régions de la Province. Certes, le produit intérieur brut par tête du Saguenay_Lac-St-Jean n'est dépassé en 1966 que par celui des Cantons de l'Est, si on ne tient pas compte de Montréal et du Nouveau-Québec. Il ne reste pas moins vrai que le taux de croissance saguenayen vient en avant-dernière position, juste avant le Nord-Ouest. C'est donc dire que la région est en perte de vitesse. Avant de tenter une explication de ce phénomène, il importe de considérer la structure de la production régionale, en tenant 
compte de l'évolution des principaux secteurs. L'intérêt d'une telle étude tient à l'idée acquise depuis longtemps à la science économique que la voie du développement passe par l'industrialisation ou, plus précisément, que la croissance économique s'accompagne généralement du déclin relatif de l'agriculture au profit de l'industrie et, ultérieurement, de l'essor du secteur des services au détriment du secteur secondaire.

Sur la base du tableau 24, on essaiera d'analyser la structure de l'économie saguenayenne et de dégager les caractéristiques de son évolution.

Tableau 24

Valeur ajoutée brute au coût des facteurs par secteurs, région 1951-1966

$\underline{\text { Retour à la table des matières }}$

\begin{tabular}{|l|r|r|r|r|c|}
\hline Secteurs & \multicolumn{2}{|c|}{$\mathbf{1 9 5 1}$} & \multicolumn{3}{c|}{$\mathbf{1 9 6 6}$} \\
\hline & $\mathbf{1 ~ 0 0 0 \$}$ & \multicolumn{1}{c|}{$\mathbf{1}$} & $\mathbf{1 0 0 0 \$}$ & $\mathbf{1}$ & Indice \\
\hline Agriculture & 15096 & 6,8 & 10177 & 2,3 & 67 \\
\hline Forêts & 23746 & 10,2 & 24178 & 4,5 & 101 \\
\hline Pêche et piégeage & 190 & 0,1 & 220 & -- & 115 \\
\hline Mines. Carrières, etc. & 1278 & 0,5 & 21870 & 4,0 & 1710 \\
\hline Fabrication & 121084 & 51,9 & 201066 & 37,0 & 166 \\
\hline Construction & 9940 & 4,3 & 24355 & 4,5 & 245 \\
\hline Transports, entreposage & 10387 & 4,4 & 19569 & 3,6 & 188 \\
\hline Communications & 1950 & 0,8 & 9014 & 1,7 & 462 \\
\hline Gaz, eau, électricité & 5345 & 2,3 & 16136 & 3,0 & 301 \\
\hline Commerce de gros & 2068 & 0,9 & 14094 & 2,6 & 682 \\
\hline Commerce de détail & 13620 & 5,8 & 51647 & 9,5 & 379 \\
\hline Finances, Assurances et Immeubles & 10462 & 4,5 & 34557 & 6,4 & 330 \\
\hline Administration publique et Défense & 3102 & 1,3 & 28431 & 5,2 & 916 \\
\hline Autres services & 14416 & 6,2 & 85256 & 15,7 & 591 \\
\hline Total & $\mathbf{2 3 2} \mathbf{6 8 5}$ & $\mathbf{1 0 0}$ & $\mathbf{5 4 0 5 7 0}$ & $\mathbf{1 0 0}$ & $\mathbf{2 3 3}$ \\
\hline
\end{tabular}

Source : A. Saicans, op. cit., pp. 39-41 


\subsection{Le secteur primaire}

$\underline{\text { Retour à la table des matières }}$

Dans ce premier secteur sont rangées généralement toutes les activités de production de produits bruts, telles que l'agriculture, les exploitations forestières et minières, la pêche et la chasse etc. Comme on peut le voir, la production globale de toutes les activités de ce secteur s'établissait à plus de 55 millions, en 1966, soit un gain absolu d'environ 16 millions de dollars par rapport à 1951 . Mais, dans le même temps, le poids relatif du secteur primaire avait enregistré un important déclin. Alors qu'en 1951, la part de ce secteur, dans l'ensemble économique régional, était de 13,7\%, en 1966, elle n'était plus que de 10,3\%, soit une baisse de 7\%. Pourtant, les activités minières, en particulier, ont connu au cours de cette période une vigoureuse expansion, due aux exploitations des riches mines de Chibougamau. La croissance du secteur minier n'a pas pu cependant compenser les pertes importantes enregistrées dans les autres secteurs, notamment de l'agriculture, dont l'apport a même diminué en valeur absolue. On peut s'interroger sur la signification de cette baisse vertigineuse du secteur primaire, qui, à première vue, et compte tenu des remarques faites plus haut, constitue un phénomène normal pour une économie en rapide expansion. Il convient, au préalable, de porter l'attention sur l'évolution encore plus singulière du secteur agricole.

Traditionnellement, l'agriculture a joué un rô1e de premier plan dans l'économie du SaguenayLac-St-Jean. Tel était le cas, tout au moins, avant le démarrage du processus d'industrialisation de cette région. En 1941 encore, le pourcentage de la main-d'œuvre occupée à des taches agricoles s'élevait à 30\% environ. Et il était encore plus élevé avant l'implantation de l'Alcan. La situation de l'agriculture de cette période reculée peut être résumée dans le tableau suivant. 


\section{Tableau 25 \\ État de l'agriculture de la région en 1921}

$\underline{\text { Retour à la table des matières }}$

\begin{tabular}{|l|r|}
\hline Superficie occupée & 801018 \\
\hline Superficie améliorée & 392863 \\
\hline Nombre de fermes & 4665 \\
\hline Grandeur moyenne des fermes & 8650 \\
\hline Superficie en blé & 78825 \\
\hline Superficie en avoine & 10950 \\
\hline Superficie en grains mélangés & 3568 \\
\hline Superficie en orge & 5191 \\
\hline Superficie en pommes de terre & 114764 \\
\hline Superficie en foin & 15,0 \\
\hline Rendement blé (boisseaux par acre) & 23,5 \\
\hline Rendement orge (boisseaux par acre) & 20,0 \\
\hline Rendement grains mélangés (boisseaux par acre) & 137,0 \\
\hline Rendement pommes de terre (boisseaux par acre) & 1,0 \\
\hline Rendement foin (boisseaux par acre) & 39139 \\
\hline Nombre total de vaches laitières & 9 \\
\hline Nombre moyen de vaches par ferme & 4391 \\
\hline Nombre de livres de lait par vache & 40716 \\
\hline Autres bestiaux & 9 \\
\hline Autres bestiaux par ferme & 13478 \\
\hline Nombre de chevaux & 58747 \\
\hline Nombre de moutons & 51984 \\
\hline Nombre de porcs & \\
\hline
\end{tabular}

Note : 1 acre $=40,5$ ares environ.

Source : BFS, Canada, cité par P.-Y. Pépin, op. cit., p. 70.

Le tableau qui précède a l'avantage de donner une vue d'ensemble de l'activité agricole dans la région, à une époque, où cette activité représentait la base fondamentale de l'économie du Saguenay-Lac-St-Jean. La superficie moyenne des fermes, relativement élevée, 172 acres, révèle une agriculture de nature largement extensive, basée sur les grandes 
cultures mécanisées et l'élevage. Pourtant, il est certain que les techniques d'alors n'étaient pas particulièrement progressives, de même que les instruments de travail. Dès lors, les rendements enregistrés ne pouvaient qu'être relativement faibles. Il n'est pas étonnant que la région devait s'en remettre au marché extérieur pour la satisfaction de ses besoins en denrées alimentaires, exception faite pour certains produits laitiers. En dépit du démarrage du processus d'industrialisation, vers 1925, et des puissants stimulants qui en découlèrent pour l'élargissement de la base agricole, les progrès restèrent lents. Certes, la marge de dépendance à l'égard de l'extérieur, pour les approvisionnements alimentaires, ne manqua pas de se rétrécir. En font foi les données du tableau 26 qui laissent apparaître de sensibles écarts entre la consommation régionale de certains produits et la production. Mais, la comparaison entre deux années caractéristiques, 1931 et 1944, permet de conclure à un progrès certain, déjà dans cette première phase de l'industrialisation régionale. Du reste, la lenteur de la croissance dans ce secteur pourrait tout aussi bien signifier que la population a préféré s'en remettre aux postes mieux rémunérés de l'industrie plut6t que de consacrer ses énergies et ressources à un développement forcené de l'agriculture, dont le poids était appelé à décliner de toute manière. Ces observations s'appuient sur les données du tableau suivant qui traduisent tout de même une certaine progression du secteur agricole régional. 
Tableau 26

Production et consommation d'aliments, SLSJ, 1944

$\underline{\text { Retour à la table des matières }}$

\begin{tabular}{|l|r|r|r|r|r|r|}
\hline & \multicolumn{2}{|c|}{ Consommation globale } & \multicolumn{2}{c|}{ Production } & \multicolumn{2}{c|}{ Déficit \% } \\
\hline Produits & \multicolumn{1}{c|}{ Livres } & \multicolumn{1}{|c|}{$\$$} & \multicolumn{1}{c|}{ Livres } & \multicolumn{1}{|c|}{} & $\mathbf{1 9 3 1}$ & $\mathbf{1 9 4 4}$ \\
\hline Bœuf & 8908000 & 1425474 & 5460155 & 873624 & 45 & 39 \\
\hline Porc & 8005087 & 1451176 & 7180121 & 1436024 & 31 & 11 \\
\hline Volaille & 2488000 & 746399 & 1100000 & 249144 & 49 & 28 \\
\hline Mouton & 715000 & 143000 & 905000 & 181037 & 4 & Exc. \\
\hline Beurre & 4633000 & 1621220 & 1001843 & 350465 & 70 & 78 \\
\hline Fromage & 500501 & 110110 & 10809044 & 2377989 & -- & -- \\
\hline Eufs & 3584000 & 1361819 & 2000000 & 648000 & 68 & 44 \\
\hline Valeur totale & & $\mathbf{6 8 5 9 6 8 9}$ & & $\mathbf{6 ~ 1 1 6 ~ 4 6 4}$ & & \\
\hline
\end{tabular}

Source : Brouillette et Dagenais, op. cit., p. 658.

À coup sûr, l'agriculture du Saguenay-Lac-St-Jean avait enregistré un développement significatif qui s'exprime par la diminution progressive des écarts entre la production des biens alimentaires essentiels et la consommation régionale de ces biens. C'était là le résultat d'une meilleure utilisation des ressources du sol, par l'application de méthodes et techniques plus rationnelles. Par exemple, la consommation d'engrais, entre 1932 et 1945, s'était multipliée par huit, passant de 350 à 2800 tonnes. Pour des années plus récentes, cette rationalisation des activités agricoles s'exprime en outre par l'élimination des petites fermes non-rentables. Ainsi, de 6439 qu'il était en 1941, le nombre de fermes est passé à 3776 en 1966, ce qui a entraîné un accroissement de 49 acres de leur superficie moyenne qui, pour la même période, est passée de 143 à 192 acres. Ce phénomène a été accompagné, comme on pouvait s'y attendre, par un élargissement continu du parc de machines agricoles. 
Tableau 27

Principaux outillages agricoles, 1951-1966

$\underline{\text { Retour à la table des matières }}$

\begin{tabular}{|l|r|r|r|}
\hline Types de machines & $\mathbf{1 9 5 1}$ & $\mathbf{1 9 6 1}$ & $\mathbf{1 9 6 6}$ \\
\hline Tracteurs & 1342 & 3383 & 3821 \\
\hline Moissonneuses-batteuses & 16 & 203 & 369 \\
\hline Presses-ramasseuses & -- & 585 & 1038 \\
\hline Moissonneuses plantes fourragères & -- & 51 & 299 \\
\hline Trayeuses électriques & 810 & 1610 & 1819 \\
\hline
\end{tabular}

Source : BFS, Canada, cité par P.-Y. Pépin, op. cit., p. 299.

La régression relative et même absolue de l'activité agricole, observée durant les quinze ou vingt dernières années dans le Saguenay-Lac-St-Jean, s'est donc effectuée en dépit du progrès observable dans les techniques et les moyens mis en oeuvre dans ce secteur. Responsable au premier chef de la chute du poids relatif du secteur primaire dans l'ensemble de l'économie régionale, elle ne peut s'expliquer que par une désaffection de la main-d’œuvre à l'égard des taches agricoles, ou de manière plus positive, par l'attrait et le pompage exercés sur cette main-d’œuvre agricole par les autres secteurs, secondaire et tertiaire, en expansion. C'est ce à quoi il faut porter maintenant l'attention.

\subsection{Le secteur secondaire}

$\underline{\text { Retour à la table des matières }}$

$\mathrm{Au}$ sens étroit du terme, le secteur secondaire se confond généralement avec les différentes branches de transformation industrielle des produits de base. Si l'on se réfère au tableau 24, il correspondrait en ce sens au secteur «fabrication », dont la valeur brute ajoutée s'élevait à 201066000 dollars, en 1966, soit 37 \% environ de la valeur ajoutée totale de l'ensemble des secteurs de l'économie saguenayenne. 
Par rapport à 1951, malgré un accroissement en valeur absolue de 80 millions de dollars, ce secteur avait décliné de façon notable, enregistrant une baisse de 15\%, en comparaison des 52\% qu'il représentait en 1951 et de son poids relatif de 37\% en 1966.

Même si on intègre la branche de la construction au secteur secondaire, comme on le fait en général, la part de ce secteur s'en trouverait gonflée d'environ 4,5\%, mais les remarques faites quant à sa relative régression resteraient valables. C'est donc que le développement de l'économie régionale s'est opéré principalement en faveur du secteur des services. Encore convient-il d'examiner les composantes de ce secteur.

\subsubsection{La structure du secteur secondaire}

On a souligné ailleurs la faiblesse caractéristique de l'industrie du Saguenay-Lac- St-Jean qui, avant l'implantation de l'Alcan, ne reposait pratiquement que sur les produits de la forêt (scieries et papeteries). Ce quasi monolithisme de la production industrielle a été brisé, quoiqu'une plus grande diversification reste peut-être encore aujourd'hui l'un des objectifs les plus souhaitables et les plus urgents pour l'industrie de la région. On peut considérer à cet effet le tableau suivant qui décrit la structure de l'industrie du Saguenay_Lac- St-Jean. 
Tableau 28

Valeur ajoutée selon les principales industries du SLSJ

$\underline{\text { Retour à la table des matières }}$

\begin{tabular}{|l|r|r|r|r|}
\hline Types d'industries & \multicolumn{2}{|c|}{1961} & \multicolumn{2}{|c|}{1965} \\
\hline Aliments et boissons & $\mathbf{1 0 0 0}$ & $\mathbf{9}$ & $\mathbf{1 0 0 0 \$}$ & $\mathbf{\%}$ \\
\hline Bois & 6675 & 3,8 & 9844 & 4,1 \\
\hline Meubles et ameublements & 6234 & 3,6 & 12395 & 5,1 \\
\hline Papiers et produits métalliques primaires & 564 & 0,3 & 720 & 0,3 \\
\hline Imprimerie, édition et industries connexes & 159059 & 90,2 & 213554 & 88,48 \\
\hline Produits métalliques & 745 & 0,4 & 1324 & 0,6 \\
\hline Produits minéraux non-métalliques & 938 & 0,5 & 1071 & 0,44 \\
\hline Autres industries & 1751 & 1,0 & 1784 & 0,7 \\
\hline Total & 397 & 0,2 & 650 & 0,28 \\
\hline
\end{tabular}

Source : Mission de Planification du SLSJ, Situation actuelle et évolution récente de l'industrie manufacturière, Ministère de l’Industrie et du Commerce, Québec août 1969, p. 48.

L'examen de la situation actuelle révèle que le secteur secondaire se concentre essentiellement sur deux importantes industries, celles du papier et des produits métalliques primaires, responsables, à elles seules, d'environ 88\% de la valeur ajoutée de l'industrie manufacturière régionale. Est-il besoin de souligner le danger que présente une telle situation, d'autant plus, comme il a été indiqué ailleurs, que le marché de la région est incapable d'absorber une portion significative de la production de ces deux industries. En effet, encore aujourd'hui, le marché extérieur reste le principal débouché du papier et de l'aluminium saguenayens. En moyenne, 63\% de ces productions s'en vont vers l'étranger, 9\% vers le reste du Canada et 28\% environ sont écoulées sur le marché provincial québécois. Les autres branches du secteur secondaire sont d'un poids relatif faible, si l'on fait exception du « Bois » qui représentait environ 5,1\% de l'ensemble industriel régional et des «Aliments et boissons », dont la part dans la valeur ajoutée totale atteignait 4,1\%, en 
1965. Fortement spécialisée, l'industrie saguenayenne ne pouvait manquer d'entraîner deux autres phénomènes : la concentration spatiale et la concentration économique.

\subsubsection{La concentration spatiale}

Le comté de Chicoutimi est responsable de plus des deux tiers de la production industrielle régionale. Selon le Bureau Fédéral de la Statistique du Canada, la valeur ajoutée totale de l'industrie manufacturière du Saguenay—Lac-St-Jean, 246592000 dollars en 1966, se répartissait comme suit :

- Comté de Chicoutimi 182338000 dollars ;

- Lac St-Jean Est 41593000 dollars ;

- Lac St-Jean Ouest 22661000 dollars.

Ces dernières données révèlent qu'une division du travail s'est opérée à l'échelle de la région, l'agriculture étant laissée aux comtés du Lac St-Jean tandis que le comté de Chicoutimi, le HautSaguenay, se spécialisait dans les activités industrielles. En 1965, Chicoutimi détenait 70\% de la main-d'œuvre industrielle régionale, soit 9846 sur les 14218 personnes travaillant dans le secteur manufacturier. Il est intéressant également de noter que plus des deux tiers de cette main-d'œuvre se trouvaient concentrés dans les quatre municipalités les plus industrialisées de la région :

- Arvida, Comté de Chicoutimi : 5915

- Alma, Lac St-Jean Est : 1828

- Kénogami, Cté de Chicoutimi. : 1425

- Port-Alfred, Chicoutimi : 791

Ce n'est donc pas sans raison que certains observateurs ont qualifié de «conurbation industrielle » la zone du Haut-Saguenay ou l'axe « Port-Alfred-Alma », par opposition à la partie, 
plus agricole, du Lac St-Jean. Mais, cette concentration de l'industrie régionale se manifeste aussi au plan économique.

\subsubsection{La concentration économique}

La concentration de l'activité industrielle du Saguenay-Lac-St-Jean se traduit par le nombre relativement minime des établissements responsables de la majeure partie de la production. Certes, «l'observation des économies contemporaines fait apparaître le rôle croissant qu'y jouent, depuis le début du XXe siècle, les grandes entreprises » 97. La concentration de l'industrie saguenayenne ne lui est donc pas particulière. Le phénomène pourrait être cependant tenu comme singulier, dans la mesure où la structure de l'économie se révélerait, à l'analyse, comme étant proche de celle des régions sous-développées. Quoiqu'il en soit, la simple observation révèle qu'un petit nombre d'établissements dominent la production industrielle du Saguenay_Lac-St-Jean, par la masse de leur valeur ajoutée, le nombre de leurs employés, les capitaux engagés ou la valeur de leurs expéditions sur les marchés extérieurs. C'est qui ressort du tableau suivant.

97 R. Barre, Économie politique, Tome 1., PUF, 1964, p. 395. 
Tableau 29

Nombre et poids relatif des établissements manufacturiers, région 1965

$\underline{\text { Retour à la table des matières }}$

\begin{tabular}{|l|c|c|c|c|c|c|}
\hline & \multicolumn{6}{|c|}{ Établissements } \\
\hline Employés & Nombre & $\mathbf{\%}$ & $\begin{array}{c}\text { Valeur ajoutée } \\
\mathbf{1 ~ 0 0 0 \$}\end{array}$ & $\mathbf{\%}$ & $\begin{array}{c}\text { Expédition } \\
\mathbf{1} \mathbf{0 0 0}\end{array}$ & $\mathbf{\%}$ \\
\hline $0-4$ & 149 & 50,0 & 1689 & 0,7 & 5284 & 1,1 \\
\hline $5-14$ & 74 & 24,7 & 4103 & 1,7 & 13931 & 3,0 \\
\hline $15-49$ & 47 & 15,8 & 6999 & 2,9 & 18900 & 4,1 \\
\hline $50-199$ & 21 & 7,1 & 17135 & 7,1 & 61707 & 13,4 \\
\hline $200-499$ & 2 & 0,7 & 17859 & 7,4 & 31885 & 6,9 \\
\hline 500 et + & 5 & 1,7 & 193555 & 80,2 & 330424 & 71,5 \\
\hline Total & $\mathbf{2 9 8}$ & $\mathbf{1 0 0}$ & $\mathbf{2 4 1} \mathbf{3 4 0}$ & $\mathbf{1 0 0}$ & $\mathbf{4 6 2} \mathbf{1 3 1}$ & $\mathbf{1 0 0}$ \\
\hline
\end{tabular}

Source : Mission de planification du Saguenay-Lac-St-Jean, op. cit., pp. 36-38.

Un simple coup d'œil sur le tableau ci-dessus indique que la production industrielle régionale repose, en majeure partie, sur sept établissements manufacturiers, employant chacun plus de 200 personnes. À elles seules, ces entreprises fournissaient, en 1965, plus de 87\% de l'ensemble de la valeur ajoutée par l'industrie régionale et près de $80 \%$ de la valeur des produits industriels vendus au-dehors. À l'opposé, une multitude de petites entreprises, 212 sur un total de 298, emploient moins de quinze personnes et ne contribuent que pour une très faible part à la production manufacturière de la région (2,4 \%). Les entreprises, employant moins de cinq personnes étaient encore d'une plus grande importance relative, quant à leur nombre, puisqu'elles représentaient près de 58 \% des établissements industriels de la région, en 1961. De manière opposée, les grandes entreprises régionales ont vu baisser leur poids relatif, quant à leur valeur ajoutée qui, par rapport au total, est passée, entre 1961 et 1965, de 90,2 à environ 88\%. Cela amène à considérer de façon plus systématique l'évolution du secteur secondaire. 


\subsubsection{L'évolution du secteur secondaire}

Du point de vue structurel, les analyses qui précèdent ont montré que l'industrie du Saguenay— Lac-St-Jean n'a subi que des changements mineurs, si on fait exception de l'importante modification apportée par l'industrie d'aluminium. Sur ce plan, l'histoire de l'industrialisation régionale s'avère relativement simple : commencée avec les scieries, elle s'est poursuivie par la transformation du bois dans les usines papetières et s'est pratiquement arrêtée avec l'aluminium. On doit cependant souligner que ces trois importantes étapes n'ont été rendues possibles que grâce au développement de la production hydroélectrique. Modeste dans le sens d'une diversification poussée, la croissance industrielle a été néanmoins relativement positive sur le plan de la consolidation des principales branches de l'industrie régionale. Cette évolution se comprend, quand on sait que le secteur proprement industriel était pratiquement quasi inexistant dans la région, dans le premier quart du XXe siècle. Dans le tableau suivant, on peut suivre le chemin de d'expansion de l'industrie régionale, dans la première période qui a suivi «la mise en place foudroyante » de la grande industrie du Saguenay—Lac-St-Jean. 
Tableau 30

Développement industriel du SLSJ 1932-1943

$\underline{\text { Retour à la table des matières }}$

\begin{tabular}{|c|c|c|c|c|}
\hline $\begin{array}{l}\text { Années } \\
\text { Région }\end{array}$ & $\begin{array}{c}\text { Capital } \\
\text { engagé en \$ }\end{array}$ & Employés & $\begin{array}{c}\text { Salaires } \\
\text { en \$ }\end{array}$ & $\begin{array}{c}\text { Production } \\
\text { valeur brute en \$ }\end{array}$ \\
\hline \multicolumn{5}{|l|}{1932} \\
\hline Chicoutimi & 57939044 & 2233 & 2221144 & 11617800 \\
\hline Lac St-Jean & 25180553 & 1010 & 935477 & 5530307 \\
\hline Région & 83119597 & 3243 & 3156591 & 17148107 \\
\hline En \% du Québec & 5,1 & 2,0 & 2,1 & 2,5 \\
\hline En \% du Canada & 1,7 & 0,7 & 0,6 & 0,8 \\
\hline \multicolumn{5}{|l|}{1940} \\
\hline Chicoutimi & 117431204 & 5531 & 7618784 & 67363580 \\
\hline Lac St-Jean & 30346410 & 1649 & 2248724 & 13934715 \\
\hline Région & 147777614 & 7180 & 9867508 & 81298295 \\
\hline En \% du Québec & 11,0 & 2,8 & 3,5 & 6,0 \\
\hline En \% du Canada & 3,6 & 0,9 & 1,1 & 1,8 \\
\hline \multicolumn{5}{|l|}{1943} \\
\hline Chicoutimi & 209202087 & 14400 & 23925212 & 179981971 \\
\hline Lac St-Jean & 38715953 & 1898 & 2769768 & 17935720 \\
\hline Région & 247918040 & 16298 & 26694980 & 197917691 \\
\hline En \% du Québec & 11,1 & 3,7 & 4,0 & 6,9 \\
\hline En \% du Canada & 3,9 & 1,3 & 1,3 & 2,3 \\
\hline
\end{tabular}

Source : Brouillette et Dagenais, op.,cit., p. 662.

Les données qui précèdent donnent un relief saisissant au rythme particulièrement rapide de la production industrielle régionale, dont la valeur a plus que décuplé entre 1932 et 1943. Relativement à la croissance générale de la Province et du pays, celle de la région a même été plus dynamique. Certes, on observe que le poids relatif de l'industrie régionale, en termes de capital engagé, du nombre d'employés, de salaires et de production, s'est multiplié par près de trois dans les deux cas. Les mêmes constatations peuvent être faites en ce qui concerne l'évolution autonome de ces quatre chefs de matière. Ce développement s'est poursuivi, même si le taux de croissance a eu tendance à diminuer dans les années plus récentes, ce qui se traduit par une 
régression relative de la région par rapport au reste de la Province et du Canada. Il importe à présent de se pencher sur la situation du secteur régional des services.

\subsection{Le secteur tertiaire}

$\underline{\text { Retour à la table des matières }}$

Même au niveau de l'ensemble du Québec, « le secteur tertiaire est mal connu du point de vue statistique et la multiplicité des activités qu'on y retrouve en rend l'étude difficile »98. Ce qui est vrai de la Province l'est davantage du Saguenay—Lac-St-Jean. Aussi l'étude du troisième secteur de l'économie régionale sera-t-elle relativement brève.

\subsubsection{Structure du secteur tertiaire}

Le secteur tertiaire, véritable fourre-tout, comprend l'ensemble des services et, de manière encore plus générale, toutes les activités qui ne sont pas directement productives. En 1966 99, la valeur ajoutée de l'ensemble de ce secteur s'élevait à 258704000 dollars, soit près de la moitié de la valeur ajoutée de tous les secteurs réunis. Il n'est peut-être pas inutile de souligner que, pour la même année, la part du tertiaire dans l'économie provinciale représentait environ $60 \%$, contre exactement $47,7 \%$, en ce qui concerne la région. Ce sont les activités commerciales, comme on pouvait s'y attendre, qui occupent la place prépondérante au sein du tertiaire régional, avec près de 66 millions de dollars de valeur ajoutée, soit plus du quart du total. Par rapport à l'ensemble de l'économie, la part de ces deux branches, « commerce de gros » et " commerce de détail », n'était pas moins de $12 \%$. À côté des services commerciaux, une place particulière doit être faite aux services des transports et des finances, dont l'apport respectif à la valeur ajoutée du secteur tertiaire montait à 15\% environ, en 1966. Mais, que le secteur tertiaire occupe une si grande place dans l'activité économique de la région, il n'en a pas toujours été ainsi, comme on va le voir.

98 Ministère de l'Industrie et du Commerce : Québec, La situation économique en 1969, p. 57.

99 Voir le tableau 24. 


\subsubsection{L'évolution du secteur tertiaire}

Contrairement aux deux premiers secteurs, primaire et secondaire, qui ont enregistré une nette régression relative, le secteur tertiaire a connu une vigoureuse expansion, consécutive sans doute au développement industriel et à son corollaire, l'urbanisation. Ainsi, de 1951 à 1966 100, la part du secteur tertiaire, en termes de valeur ajoutée, est passée de 26,2 à 47,7\% de la valeur ajoutée de l'économie régionale. Pour des années plus reculées, l'exemple de l'évolution du secteur commercial parait significatif de cette rapide expansion.

Tableau 31

Le commerce de détail, région 1931-1941

$\underline{\text { Retour à la table des matières }}$

\begin{tabular}{|l|c|c|c|}
\hline & $\begin{array}{c}\text { Ventes en \$ } \\
\mathbf{1 9 3 1}\end{array}$ & $\begin{array}{c}\text { Ventes en \$ } \\
\mathbf{1 9 4 1}\end{array}$ & $\begin{array}{c}\text { Accroissement } \\
\text { en pourcentage }\end{array}$ \\
\hline Cité de Chicoutimi & 7058900 & 18404300 & 160,7 \\
\hline Citées du Lac St-Jean & 3690400 & 7648200 & 107,2 \\
\hline Région & 10749300 & 26052500 & 143,3 \\
\hline
\end{tabular}

Source : Brouillette et Dagenais, op. cit., p. 675.

De 1931 à 1941, le commerce de détail de la région a plus que doublé, passant de 10749 300 dollars à 26052500 dollars. Cette singulière expansion indique clairement que le secteur tertiaire s'est développé au détriment du secteur primaire, et ce qui est moins normal, du secteur secondaire. À première vue, l'évolution structurelle de l'économie du Saguenay_Lac-St-Jean, tout en relevant du phénomène général de la croissance, présenterait certains aspects pathologiques. Encore convient-il, pour en juger, d'analyser préalablement d'autres éléments tout aussi importants, eu égard à la croissance économique.

100 Voir le tableau 24. 


\section{Section 2 : Le revenu et le niveau de vie}

$\underline{\text { Retour à la table des matières }}$

11 est presque banal de dire que le premier objectif de tout système économique est la recherche du mieux-être pour les hommes, une meilleure satisfaction de leurs besoins. Sans préjuger de l'échelle des valeurs d'une collectivité, on peut donc conclure que le caractère progressif ou la croissance de l'économie d'une région doit se traduire par la hausse du niveau de vie de ses habitants et l'extension à des couches de plus en plus larges de la population des avantages du progrès. À cet effet, dans les économies modernes de marché, l'évolution du revenu disponible, en dépit de ses inconvénients, peut être considérée comme un critère de choix. Certes, on peut admettre que « le concept de niveau de vie, mesuré par le revenu per capita, ne permet pas d'internaliser les effets externes de la croissance, positifs ou négatifs, qui se reflètent par contre sur les conditions de vie » 101. Il n'en reste pas moins vrai cependant que ce concept est pratiquement irremplaçable quand il s'agit de présenter une vue synthétique de ces phénomènes multiples, dont l'examen, même superficiel, déborderait le cadre de cette étude. C'est donc à l'analyse du revenu que seront consacrés les développements qui suivront. Parallèlement, on examinera les problèmes connexes relatifs à la main-d’œuvre et à la population.

\subsection{Le revenu : évaluation et évolution}

Le revenu disponible, dans le Saguenay—Lac-St-Jean, a suivi de près la courbe d'évolution des activités économiques régionales. Sur une base de 100, en 1951, l'indice du revenu disponible par tête est passé à 200 en 1966, comparativement, sur la même base, contre 196 pour l'ensemble de la Province du Québec. Cette évolution, relativement favorable, a quelque peu contribué à combler l'écart traditionnel entre le revenu moyen de la région et celui de l'ensemble

101 N.V. Martin, «L'intégration de l'économique et du social dans une politique axée sur le développement » dans Le Québec d'aujourd'hui, regards d'universitaires, HMH, Hurtubise, Montréal, 1971, p. 176. 
québécois. L'on doit cependant faire observer que la progression reste, en ce domaine, plutôt lente, et que la différence entre ces deux variables est encore aujourd'hui très significative. Certes, en 1966, le revenu disponible par tête de la région n'était que de 1380 dollars, comparativement à 1705 dollars pour le Québec. L'examen du tableau qui suit révèle tout le chemin qu'il reste à parcourir au Saguenay_Lac-St-Jean pour se hisser au niveau des régions les plus favorisées de la Province.

\section{Tableau 32 \\ Revenu disponible par tête en dollars Province de Québec et régions, 1951-1966}

$\underline{\text { Retour à la table des matières }}$

\begin{tabular}{|l|c|r|r|r|}
\hline \multicolumn{1}{|c|}{ Territoires } & $\mathbf{1 9 5 1}$ & $\mathbf{1 9 5 6}$ & $\mathbf{1 9 6 1}$ & $\mathbf{1 9 6 6}$ \\
\hline Bas St-Laurent Gaspésie & 499 & 653 & 765 & 1155 \\
\hline Saguenay-Lac-St-Jean & 691 & 888 & 1001 & 1380 \\
\hline Québec (région) & 714 & 839 & 1073 & 1443 \\
\hline Trois-Rivières & 745 & 854 & 1076 & 1430 \\
\hline Cantons de l'Est & 745 & 818 & 1096 & 1490 \\
\hline Montréal (région) & 1032 & 1266 & 1480 & 1938 \\
\hline Outaouais & 775 & 854 & 1037 & 1413 \\
\hline Nord-Ouest & 727 & 845 & 1004 & 1372 \\
\hline Côte-Nord et Nord du Québec & 879 & 1188 & 1037 & 1677 \\
\hline Ensemble provincial & 870 & 1057 & 1270 & 1705 \\
\hline
\end{tabular}

Source : A. Saicans, Rapport pour l'OPDQ, 1969, p. 61.

Comme on le voit, sur les dix régions composant le Québec, le Saguenay_Lac-St-Jean vient au huitième rang, avant le Nord-Ouest et le Bas St-Laurent - Gaspésie, pour le revenu disponible per capita. Il est pourtant un fait reconnu que la région du Saguenay bénéficie de l'un des niveaux de revenu disponible par unité de main-d’œuvre les plus élevés de la Province. Ces deux ordres de réalité ne sont pas vraiment contradictoires, comme le fera ressortir la suite de l'analyse. 


\subsection{La structure du revenu}

$\underline{\text { Retour à la table des matières }}$

L'analyse du revenu par grands secteurs de production apporte une nouvelle confirmation du déclin de l'agriculture dans l'ensemble de l'économie du Saguenay_Lac-St-Jean et l'accroissement simultané du poids relatif des salariés, notamment de l'industrie. Considérons à cet effet le tableau de la composition du revenu disponible.

Tableau 33

Structure de revenu disponible Saguenay_Lac-St-Jean, 1951-1966

\begin{tabular}{|l|c|r|r|c|}
\hline & \multicolumn{2}{|c|}{1951} & \multicolumn{2}{c|}{$\mathbf{1 9 6 6}$} \\
\hline \multicolumn{1}{|c|}{ Catégorie d'agents } & $\mathbf{1 ~ 0 0 0 \$}$ & $\mathbf{~ \%}$ & $\mathbf{1 0 0 0 \$}$ & $\mathbf{\%}$ \\
\hline Revenu des salariés & 97774 & 68,6 & 293865 & 70,2 \\
\hline Revenu de l'agriculture & 13409 & 9,4 & 6581 & 1,6 \\
\hline Revenu des entreprises & 11050 & 7,7 & 28722 & 6,9 \\
\hline Intérêts, dividendes, etc. & 5096 & 3,6 & 20383 & 4,9 \\
\hline Revenu total & 142571 & 100 & 418239 & 100 \\
\hline Moins impôts & $(3557)$ & & $(28998)$ & \\
\hline Revenu disponible & 139014 & & 389241 & \\
\hline
\end{tabular}

Source : A. Saicans, op. cit., page 58.

Un simple coup d’œil sur le tableau qui précède laisse apparaître l'importance de la classe des salariés dans la répartition du revenu disponible de la région. Avec 70,2\% de cette masse, ils ont incontestablement la part du lion. Du reste, cette catégorie de travailleurs a eu la faveur de l'évolution, puisque leur part du revenu disponible a enregistré une augmentation de 1,6\% entre 1951 et 1966, contrairement aux travailleurs du secteur agricole. Ces derniers ont en effet enregistré une baisse de leur part du revenu disponible, soit près de $8 \%$. À la vérité, les trois principales catégories d'agents économiques, prises ensemble, les salariés, les agriculteurs et les 
entrepreneurs, ont subi une assez forte détérioration dans leur part du revenu disponible régional, qui est passée de 85,7 à 78,7\%, de 1951 à 1966. Ce sont ainsi les revenus de transfert qui ont enregistré le plus fort accroissement. Dans la mesure où ces revenus ne correspondent généralement à aucune création de biens ou de services, leur expansion démesurée est significative d'une situation qui n'est pas réjouissante. En effet, ce phénomène peut s'identifier à un accroissement du nombre des sans-travail et donc d'une augmentation de la charge pesant sur les éléments productifs de la collectivité. Cette considération amène à examiner le revenu par rapport à la main-d’œuvre et à la population.

\subsection{Population, main-d'œuvre et revenu}

$\underline{\text { Retour à la table des matières }}$

L'analyse de la population par rapport à la main-d'œuvre revêt une assez grande importance, dans le cadre de l'examen du revenu d'une région. En effet, le revenu produit par les travailleurs contribue à faire vivre l'ensemble de la collectivité, y compris ceux qui, pour une raison ou une autre, ne sont pas productifs. Or, on a déjà indiqué ailleurs que l'une des caractéristiques essentielles de la population du Saguenay_Lac-St-Jean est sa relative jeunesse. D'autre part, le caractère fondamental d'économie extravertie fait que la région est particulièrement sensible au phénomène du chômage, assez général, du reste, dans la province du Québec. À l'appui de cette dernière remarque, l'Office de Planification et de Développement du Québec souligne que « la région du Saguenay_Lac-St-Jean a été aux prises avec un taux de chômage moyen de 14\% entre 1951 et 1965, les fluctuations saisonnières étant de l'ordre de 5\% par rapport à cette moyenne. Une estimation du chômage régional, poursuit l'office, indique qu'en 1969 ce taux n'a pas changé : 11 000 personnes sans emploi sur une main-d’œuvre de 79000 personnes » 102.

Ces deux facteurs combinés, jeunesse de la population et chômage, expliquent que le poids reposant sur la main-d’œuvre est particulièrement élevé. En 1961, un travailleur du Saguenay-

102 OPDQ, Saguenay-Lac-St-Jean, esquisse de plan de développement, Québec, décembre 1969, p. 31. 
Lac-St-Jean devait faire vivre en moyenne 3,71 personnes. Pour la même année, le rapport était de 1 pour 2,89 personnes dans la Province du Québec et de 1 pour 2,10 environ à Toronto 103. Ces données restent largement valables encore aujourd'hui. Les observations qui précèdent éclairent le paradoxe dont il a été question plus haut, à savoir la faiblesse relative du revenu moyen disponible au Saguenay_Lac-St-Jean. Il est vrai que cette région bénéficie de l'une des moyennes de salaires les plus élevées de la Province, notamment en ce qui concerne les activités des secteurs secondaire et tertiaire.

\section{Tableau 34}

Zone urbaine de Chicoutimi et Province

(salaires hebdomadaires moyens en dollars)

$\underline{\text { Retour à la table des matières }}$

\begin{tabular}{|l|c|c|c|c|c|}
\hline & \multicolumn{5}{|c|}{ Chicoutimi } \\
\hline Années & Industrie & Construction & Services & Moyenne & Moyenne Québec \\
\hline 1957 & 85,38 & 69,88 & 79,86 & 79,92 & 65,22 \\
\hline 1959 & 93,56 & 79,70 & 91,53 & 87,99 & 70,55 \\
\hline 1961 & 105,07 & 94,28 & 96,20 & 96,39 & 75,67 \\
\hline 1963 & 111,09 & 91,42 & 95,35 & 100,52 & 80,99 \\
\hline 1965 & 117,22 & 91,94 & 108,78 & 105,51 & 88,62 \\
\hline 1968 & 140,68 & 108,41 & 119,48 & 125,13 & 107,86 \\
\hline
\end{tabular}

Source : BFS, Canada : Average Weekly Wages and Salaries.

L'examen du tableau précédent permet de voir qu'en moyenne les salaires des travailleurs des zones urbaines de la région $(125,13 \$)$ dépassent largement deux de la province du Québec $(107,86 \$)$ et même ceux de l'agglomération montréalaise (non-représentés : 109,60 dollars en 1968). Les hauts salaires versés par l'industrie de l'aluminium et celle du papier sont responsables de cette situation. Ils ne compensent pas, du reste, la faiblesse du niveau de revenu des autres secteurs moins riches de l'économie régionale. Ainsi, en 1961, le revenu personnel par unité de maind’œuvre de la région, 3616 dollars, ne représentait qu'environ 92\% de cette même variable, au niveau 
provincial. Mais, on peut noter que, la même année, le revenu personnel per capita de la région, un peu plus de mille dollars, n'atteignait que 78,8\% de celui du Québec.

En dépit de ces désavantages relatifs, il ne fait pas de doute que le Saguenay—Lac-St-Jean a connu une croissance relativement soutenue et que ses habitants peuvent bénéficier aujourd'hui de tous les avantages de la vie moderne. Incontestablement, le niveau de vie et le degré de bien-être de la collectivité régionale se sont largement améliorés. Une fine analyse de l'évolution de la consommation régionale, au double point de vue quantitatif et qualitatif, confirmerait, à coup sûr, cette assertion, mais déborderait, par trop, le cadre de cette étude. Certes, il n'est pas possible d'analyser ici les multiples composantes du niveau de vie des collectivités saguenayennes qui ont accédé à ce qu'on convient d'appeler « les sociétés de consommation », encore moins d'étudier le passage de ces populations de la vie rurale, simple, à la vie urbaine, axée sur le confort matériel. $\mathrm{Au}$ passif de cette évolution, il faudrait analyser aussi les vains espoirs qu'a fait naître l'industrialisation et, sans doute, également, la détérioration relative de l'environnement. Si le premier aspect négatif de ce mouvement, les désillusions de l'industrialisation, est plus grave, se traduisant par un exode massif des jeunes vers l'extérieur 104, le second, le déséquilibre du système écologique, reste insignifiant, compte tenu du nombre et de l'immensité des sites naturels non encore touchés par la pollution. Reste à déterminer le rôle joué par l'Alcan dans cette évolution de la région.

104 Note : selon l'OPDQ, la région a connu un bilan migratoire négatif de l'ordre de 22000 personnes, entre 1961 et 1966. 


\section{TROISIÈME PARTIE}

\section{Le rôle moteur de l'Alcan}

$\underline{\text { Retour à la table des matières }}$

À cette étape de l'étude, il importe de dépasser le caractère partiel et autonome qu'on a délibérément imprimé jusque-là à l'analyse des principales variables de l'économie saguenayenne, pour essayer de les saisir dans la dynamique de leur interaction réciproque. De manière plus explicite, il s'agira de préciser la nature du rôle joué par l'Alcan dans l'évolution fondamentale de l'économie du Saguenay—Lac-St-Jean.

Certes, il n'échappe plus à l'attention du lecteur averti le poids de l'influence qu'une société, comme l'Alcan, est susceptible d'avoir sur une économie. À cet effet, la présentation de la Compagnie, dans sa triple dimension, ainsi que le tracé de l'évolution de l'économie de la région n'ont pas manqué d'être significatifs. Même, on n'a pu s'empêcher, au cours de cette phase de la recherche, de laisser apparaître certains éléments de cet aspect essentiel. Désormais, il conviendra de faire l'analyse, plus ou moins systématique, de cette action de l'Alcan, en dégageant ses composantes fondamentales. Cette démarche indispensable devrait permettre de voir dans quelle mesure l'influence de l'entreprise peut 
être qualifiée de motrice ou de polarisante, à l'égard de la région. Or, s'il est vrai, comme le souligne Rosenfeld que «seul l'établissement d'une comptabilité économique régionale détaillée permet de reconnaître les entreprises qui assurent un rôle moteur dans le développement économique d'une région, de mesurer l'intensité de leurs effets d'entrainement sur les différents secteurs et de déterminer le degré de dépendance de ceux-ci à l'égard des firmes motrices » 105, on ne cachera pas le caractère malaisé de cette étape de la recherche. À la vérité, il faut convenir, dès l'abord, que cette tâche ne pourra être que partiellement réussie, étant donné l'inexistence d'une comptabilité économique régionale détaillée. Certes, les matériaux disponibles permettront de mettre en évidence et d'analyser les aspects positifs de l'influence de l'Alcan sur le Saguenay_Lac-St-Jean. Plus difficile sera la tâche complémentaire, d'identifier et d'analyser les effets négatifs. Mais, qui ne sait que «toute médaille a son revers » et que le phénomène de polarisation se caractérise aussi bien par l'attraction que par la répulsion, ou, que l'expansion inhérente à la croissance économique crée inévitablement vide et déséquilibre ? Sans être péremptoire, mais sans complaisance, on tentera donc d'analyser quelques hypothèses quant au caractère négatif de l'action de l'Alcan sur l'économie saguenayenne. Cela permettra d'établir un bilan concernant le râle de cette société dans la croissance économique régionale.

On est ainsi conduit à diviser cette troisième partie de l'étude en deux chapitres. Dans le premier, on verra les effets positifs de l'Alcan sur l'économie du Saguenay_Lac-St-Jean et dans le second, on étudiera les limites du processus de développement régional à partir de l'Alcan.

105 F. Rosenfeld, «Les firmes motrices et la comptabilité régionale », dans Les Cahiers de L'ISEA, no 11, octobre 1962, p.71. 
Troisième partie : Le rôle moteur de l'Alcan

\section{Chapitre VI}

\section{Les effets de l'Alcan sur le Saguenay-Lac-St-Jean}

$\underline{\text { Retour à la table des matières }}$

En vue d'établir les répercussions d'une entreprise sur un ensemble économique plus vaste, on peut faire appel à diverses méthodes qui se ramènent fondamentalement à deux : la méthode dite opérationnelle et quantitative et la méthode qualitative et fonctionnelle. Si la première, qui se base sur le modèle «input-output » de Léontief, a l'avantage de la rigueur et de la précision mathématique, la seconde reste plus facilement applicable, étant donné la pauvreté et l'hétérogénéité des matériaux statistiques généralement disponibles sur les régions. Les deux méthodes sont du reste largement complémentaires. Pour question de commodité, c'est la seconde méthode qui sera utilisée ici. Quoiqu'il en soit, l'impact économique de tout projet spécifique de développement s'exerce à travers des effets directs, indirects et induits 106. Cette nomenclature classique constituera la trame de l'analyse de l'action de l'Alcan sur l'économie du Saguenay—Lac-St-Jean.

106 F. Rosenfeld, Les firmes motrices et la comptabilité régionale, op. cit., pp. 78-79. 


\section{Section 1 : Les effets directs de l'Alcan}

$\underline{\text { Retour à la table des matières }}$

Par « effets directs », on entendra « la valeur ajoutée du nouvel ensemble dans l'économie de la région d'implantation » 107. C'est en quelque sorte la production totale de l'entreprise ou encore son apport direct à la communauté régionale de toutes les catégories de revenus provenant de ses activités. Mais, qui dit production ou création de revenus dit mise en oeuvre préalable de facteurs de production. C'est donc au niveau des investissements et de l'emploi que les premiers effets du nouvel ensemble se feront sentir.

\subsection{L'Alcan et les investissements régionaux}

L'importance des investissements pour une économie n'est pas à démontrer. Générateur de revenu et créateur de capacité, l'investissement est à la base de la croissance économique. Il est évident que, dépendant du secteur d'activité où ils s'effectuent, les investissements présentent divers degrés d'efficience qui ne coïncident pas forcément avec leur rentabilité propre. Mais, on peut admettre le point de vue du Professeur Hirschman, selon lequel « une caractéristique du développement, fondamentale pour l'analyse comme pour la stratégie, engage moins à trouver des combinaisons optimales de ressources et de facteurs de production donnés qu'à faire apparaître et mobiliser des ressources et des capacités cachées, éparpillées ou mal utilisées » 108.

Dans la perspective définie précédemment, les projets d'implantation de l'Alcan au Saguenay_Lac-St-Jean ont entraîné des effets bénéfiques pour l'économie de cette région, dont les immenses ressources latentes demeuraient sous-exploitées jusqu'en 1925. On a présenté ailleurs 109 l'historique de l'implantation de la Société. Sans vouloir faire ici le complet

107 F. Rosenfeld, ibid, p. 78.

108 A. O. Hirschman, op. cit., p. 17.

109 Voir la deuxième partie, le chapitre 3. 
inventaire des immobilisations saguenayennes de l'Alcan, il convient de rappeler les principales entreprises de cette compagnie dans la région.

Au premier chef, il faut citer le complexe électro-métallurgique d'Arvida, le plus important centre de production d'aluminium de première fusion du monde. Vient ensuite l'usine d'IsleMaligne, spécialisée également dans la fabrication de l'aluminium. À côté de ces activités de base, la Société exploite six grandes centrales hydroélectriques, dont la capacité dépasse 2500 $000 \mathrm{kWh}$, un port moderne dont on a souligné l'importance pour l'économie régionale, PortAlfred, et deux lignes de chemin de fer. Vers la fin de 1969, selon les responsables de la Compagnie, la valeur des immobilisations de l'Alcan dans le Saguenay_Lac-St-Jean montait à 650 millions de dollars environ, soit un tiers de l'ensemble des investissements régionaux. Ces données traduisent, en ce qui a trait au financement de la croissance, la très grande importance de l'Alcan pour l'économie du Saguenay—Lac-St-Jean.

Il va sans dire que ces investissements ont été effectués sur une très longue période. La Société comptera bientôt un demi-siècle d'existence dans la région. Mais, la majeure partie des immobilisations régionales de l'Alcan ont été mises en place durant la période s'étendant entre 1925-27 et le milieu de la seconde guerre mondiale. On doit signaler également l'érection de trois centrales hydroélectriques, totalisant environ 1165000 kWh, entre 1952 et 1960.

De l'avis de certains observateurs, l'Alcan semblerait avoir atteint un certain seuil de maturité. Cela signifie que la région ne devrait plus attendre grand chose de la part de la Société dans le domaine des investissements. Ainsi souligne P.-Y. Pépin, «la croissance véritable est terminée pour l'Alcan, même si des capitaux doivent être constamment injectés afin de rationaliser davantage l'appareil de production » 110. Certes, sauf circonstances exceptionnelles entraînant une poussée forte et durable de la demande mondiale d'aluminium, l'expansion au terme de laquelle l'Alcan a fait du Saguenay_Lac-St-Jean l'une des régions productrices d'aluminium du globe semble arrêtée. De là à conclure que la croissance véritable de l'Alcan dans la région est terminée est quelque peu hâtif. En effet, rien n'interdit à la Compagnie, contrôlant désormais l'ensemble des

110 P. -Y. Pépin, op. cit., p. 404. 
opérations aboutissant à l'aluminium de première fusion, d'orienter ses activités régionales dans le sens de la diversification de son output. Dans une perspective de long terme, il pourrait même y aller de son intérêt. Comme le souligne H.D. Wallace, « the problem of achieving the best balanced structure... involves the best utilization of business ability through the extension of control horizontally, vertically and laterally, i.e. over the making of commodities other than aluminum which are produced with aluminum or electric energy or both, in such way that marginal product is the same in each direction » 111. Certains indices laissent penser que l'Alcan pourrait effectuer bientôt un virage dans cette direction. Il est significatif, en effet, que depuis 1966 la majeure partie des investissements régionaux de la Société, 850000 dollars environ, aient été consacrés à l'élargissement des opérations de son usine de laminoir à tiges d'Arvida. Plus significatif encore a été le lancement, au début de 1972, d'un important centre de relaminage qui a nécessité plus de quatorze millions de dollars d'investissement et qui fournit de l'emploi à 70 personnes. Ce sont là, on en conviendra, d'importantes étapes sur le chemin de la transformation de l'aluminium en produits finis. Cette question sera analysée dans le cadre de la deuxième section du présent chapitre. Pour le moment, il convient d'examiner les effets de l'Alcan sur la main-d'œuvre du Saguenay-Lac St-Jean.

\subsection{L'Alcan et l'emploi régional}

$\underline{\text { Retour à la table des matières }}$

Au départ, il faut souligner que la fabrication de l'aluminium, activité de forte intensité capitalistique, ne réclame qu'une main-d'œuvre quantitativement restreinte. Selon Gadeau, « il faut en moyenne 16 heures de travail par tonne d'aluminium, en comptant la fabrication, l'entretien et la réfection des cuves et, en plus, deux heures par tonne pour la fonderie et la coulée en lingots » 112. Pour sa part. G. Blardone note que « de manière assez générale, l'électrométallurgie est à faible coefficient d'emploi » 113.

111 H.D.Wallace, op. cit., p. 188.

112 R. Gadeau, L'aluminium, A. Colin, Paris, 1958, p.35.

113 G. Blardone, Encyclopédie française, tome IX, p. 9-36-16. 
Il ne fait aucun doute que la hausse continue de la productivité du travail découlant du progrès technique contribue encore à la baisse progressive du coefficient d'emploi, en général. Par ailleurs, en ce qui concerne l'Alcan, dont les activités régionales sont largement tributaires du marché international, l'emploi créé ne peut être que très sensible à la conjoncture mondiale. Les dangers d'une telle situation sont trop évident pour qu'il soit nécessaire d'insister là-dessus, d'autant plus que, comme il a déjà souligné, l'Alcan pèse très lourd dans la balance de l'activité économique générale du Saguenay_Lac-St-Jean.

On ne dispose pas de données précises quant au volume de l'emploi créée par l'Alcan dans la phase de son implantation dans la région et au poids relatif de ce personnel par rapport à la maind'œuvre régionale globale. Ces données sont du reste d'un intérêt mineur, étant donné que ce personnel, travaillant soit à l'érection des barrages ou à la mise en place d'autres infrastructures, soit à la construction de l'usine d'aluminium et de la ville d'Arvida, était appelé à se reconvertir, une fois ces travaux achevés. On estime cependant qu'il devait être environ au nombre d'un millier, la plupart venant de l'extérieur. «Commencés en septembre 1925, les travaux, souligne R. Blanchard, furent menés très vite avec des centaines d'ouvriers de toutes nationalités (Polonais, Suédois, Norvégiens, Finlandais, Italiens, etc.) ». 114

L'ensemble des travaux d'installation terminés, l'emploi résiduel fut, semble-t-il, relativement important. En effet, en 1926, la nouvelle usine d'aluminium commença à fonctionner avec un personnel estimé à 800 travailleurs, nombre qui devait passer à une moyenne annuelle de 1650 sur les deux années suivantes. La crise des années 1930 se répercuta de manière brutale sur les activités de l'usine d'Arvida, dont le personnel tomba brusquement à 400 unités, vers 1931. Même alors, l'apport de l'Alcan à la région, en matière de création d'emplois, était non négligeable, si l'on en croit Blanchard soulignant que durant cette période de marasme économique « le personnel de l'industrie proprement dite, travaillant à pleine capacité, était au total de 6500 hommes environ » 115. C'est au milieu de la seconde guerre mondiale, vers 1943, que l'Alcan connut, pour ainsi dire, l'apogée de ses activités. Le personnel à son service atteignit alors le chiffre

114 R. Blanchard, L’Est du Canada français, op. cit., p. 105.

115 R. Blanchard, idem, p. 106. 
record de 11800 personnes, concentrées particulièrement dans le comté de Chicoutimi, ainsi qu'il apparaît au tableau suivant.

Tableau 35

Répartition géographique du personnel de l’Alcan, région 1943

$\underline{\text { Retour à la table des matières }}$

\begin{tabular}{|l|c|c|}
\hline Localité & Comté & Effectifs \\
\hline Arvida & Chicoutimi & 4400 \\
\hline Jonquière et Kénogami & Chicoutimi & 4000 \\
\hline Chicoutimi (agglom.) & Chicoutimi & 2750 \\
\hline Isle-Maligne & Lac-St-Jean & 550 \\
\hline Ailleurs & & 100 \\
\hline Total région & & 11800 \\
\hline
\end{tabular}

Source : Brouillette,B., article cité, page 432.

En 1941, la main-d’œuvre totale recensée dans le Saguenay-Lac-St-Jean s'élevait à 43313 personnes, répartie entre le comté de Chicoutimi (24 891), et les comtés du Lac St-Jean, (18 422 ). Par suite d'une progression relativement rapide entrainée par les besoins de l'économie de guerre, le volume de population active régionale passait à 65000 personnes environ en septembre 1943. Ainsi, au cours de cette période, la contribution directe de l'Alcan à la création d'emplois n'était pas moins de 18 \% de la main-d’œuvre régionale. Elle l'était davantage, à la vérité, considérée par rapport aux effectifs proprement industriels.

Certes, le poids relatif de l'Alcan dans ce domaine a subi dans le temps une certaine régression. Aujourd'hui, la main-d'œuvre totale employée par les entreprises régionales de la Compagnie s'est stabilisée plus ou moins autour de 9000 personnes, soit environ 14\% de l'emploi régional. En 1969, pour une population active estimée à 79000 personnes, il y avait en effet 68000 unités effectives d'emploi, dont 15\% dans le secteur primaire, 22\% dans le secteur secondaire et 63\% dans 
le secteur tertiaire 116. Comparée à la structure de la main-d’œuvre régionale des années 1925-26, la composition actuelle de la main-d'œuvre révèle une évolution effectuée dans le sens d'une certaine industrialisation de l'économie. L'influence de l'Alcan sur cette évolution a été déterminante : le personnel de la compagnie, concentrée principalement dans le secteur secondaire, représente certes près de $40 \%$ des effectifs industriels régionaux. Cependant, le taux particulièrement élevé du chômage qui sévit dans la région allié au poids relativement faible du secteur secondaire de l'économie montre l'étendue des progrès encore susceptibles d'être réalisés.

Aux effets quantitatifs et structurels de l'Alcan sur la main-d'œuvre du Saguenay—Lac-StJean, il faut ajouter les modifications qualitatives qui n'ont pas manqué de se produire. Il est vrai, comme on l'a souligné plus haut, que dans les premiers temps de son fonctionnement, l'entreprise fit appel à l'extérieur pour l'obtention d'une bonne partie de son personnel, notamment les cadres techniques et administratifs. Mais, progressivement, ces derniers se sont intégrés à l'univers régional. En outre, la présence des usines de l'Alcan, comme débouché pour la main-d'œuvre locale, a été un puissant stimulant pour la qualification professionnelle. De fait, aujourd'hui, la majeure partie du personnel au service de l'Alcan est puisé dans le réservoir de la main-d'œuvre régionale qui s'est ainsi adaptée aux exigences de la grande industrie moderne. Il est significatif que la productivité moyenne du secteur secondaire régional soit supérieure à celle du Québec, respectivement 8,71 contre 5,35, en 1961 117. C'est, avec les papeteries, l'industrie d'aluminium qui est le principal responsable de ce phénomène. La productivité des travailleurs de cette industrie est en effet la plus élevée, comparativement à celle des autres secteurs de l'économie de la région. Il n'est pas dès lors étonnant qu'ils bénéficient des niveaux de salaires les plus intéressants de la région.

116 OPDQ, Mission de planification régionale au SLSJ, esquisse de plan de développement, 1969, p. 66.

117 OPDQ, op. cit., p. 66. 


\subsection{L'Alcan et les revenus régionaux}

$\underline{\text { Retour à la table des matières }}$

On a défini précédemment les effets économiques directs d'un complexe industriel sur son milieu d'implantation comme étant l'ensemble de sa valeur ajoutée. De manière plus explicite, ils se mesurent à la somme « des salaires distribués, des charges sociales correspondantes, des impôts payés dans la région et du bénéfice brut (amortissement compris) retenu ou distribué sur place » 118. En somme, il s'agit de l'impact du complexe considéré sur la création et la distribution de revenus dans la région.

En ce qui concerne les effets directs de l'Alcan sur le Saguenay-Lac-St-Jean, il est presque superflu de souligner qu' ils ne se confondent nullement avec l'ensemble de la valeur ajoutée de ses entreprises. On le comprend bien, si l'on se rappelle que cette Société est essentiellement une grande unité inter-territoriale.

En effet, de quelle utilité pourrait être à la région cette partie de la valeur ajoutée servant à libérer l'Alcan de ses engagements financiers, contractés le plus souvent sur le marché canadien et même international ? En outre, quelque soit le volume des bénéfices réalisés, il est certain que leur influence sur l'économie saguenayenne est pratiquement nulle, vu qu'ils vont en majeure partie, sous forme de dividendes à des actionnaires résidant hors de la région. Ces remarques, à elles seules, justifieraient, pour une évaluation précise des effets directs de l'Alcan sur le Saguenay_Lac-St-Jean, l'élaboration d'un tableau de ventilation des recettes de toutes les entreprises régionales de la Compagnie selon les chefs et les centres de leur utilisation. L'indisponibilité de données sur ce sujet rend pareille démarche impossible. Néanmoins, sans crainte de se tromper, on peut avancer que, mises à part les sommes engagées dans les investissements régionaux, les salaires distribués et

118 F. Rosenfels, op. cit., p. 78. 
les impôts locaux payés par l'Alcan constituent l'essentiel de ses effets directs sur l'économie de la région.

\subsubsection{Les salaires}

Relativement à la valeur ajoutée de l'ensemble du secteur manufacturier du Saguenay—LacSt-Jean, celle des entreprises de fabrication d'aluminium est énorme. En 1965, sur la somme de 241339200 dollars représentant le total de la valeur ajoutée par les diverses branches industrielles de la région, la part de l'industrie d'aluminium s'élevait à 144552600 dollars, soit plus de la moitié. On peut noter que pour la même année la valeur ajoutée par unité de main-d’œuvre dans cette industrie s'élevait à 21200 dollars contre une moyenne de 17100 dollars pour tout le secteur industriel régional. Cette moyenne, du reste, tombe à seulement 7700 dollars, si on ne tient pas compte de l'apport spécifique des industries d'aluminium et du papier.

De manière évidente, une partie importante de la masse de cette valeur ajoutée, versée à l'extérieur, n'entraine pas des avantages directs pour le Saguenay_Lac-St-Jean. Mais, on doit souligner qu'en 1965, près d'un tiers de cette valeur ajoutée était versée à la région, sous forme de salaires, soit environ 45500000 dollars, représentant 55\% des salaires industriels régionaux. Compte tenu de la haute productivité des travailleurs de l'industrie d'aluminium, cette branche vient au premier rang pour le salaire versé par unité de main-d’œuvre employée, soit, en 1965, 6100 dollars contre une moyenne de 4400 dollars pour l'ensemble du secteur manufacturier régional.

À ces revenus, dont limpact sur l'économie de la région ne saurait faire de doute, il faut encore ajouter tous les bénéfices marginaux dont jouit le personnel au service de la Compagnie. L'impossibilité de leur comptabilisation n'enlève rien à leur influence. Ils sont représentés par les sommes versées par la Société à titre de participation à la sécurité sociale des travailleurs, au fonds de pension de vieillesse, au financement de services à prix modiques aux employés, comme les restaurants, les stages de formation ou de recyclage. L'Alcan accorde en outre un certain nombre de bourses d'études ou divers prêts d'honneur à des jeunes de la région : 36 bourses, en 1968, à des étudiants de différentes universités canadiennes et environ 30000 dollars au Fonds 
Alcan de la Société St-Jean-Baptiste. Ces contributions, apparemment dérisoires, n'entrainnent pas moins des effets bénéfiques pour l'économie du Saguenay—Lac-St-Jean.

\subsubsection{Les impôts}

On ne peut ignorer aujourd'hui le rôle majeur dévolu à l'État dans le fonctionnement de la vie économique et sociale des collectivités modernes. Que ce soit par la mise en place ou l'amélioration des infrastructures, par le maintien des institutions ou leur ajustement permanent aux exigences du progrès, les corps publics constitués représentent sans doute les agents les plus importants des sociétés contemporaines. Or, c'est de limpôt, sous toutes ses formes, qu'ils tirent l'essentiel des ressources propres à financer la réalisation de leurs taches, multiples et vitales. L'on reconnaît dès lors l'importance que revêt la participation, souvent majeure en fonction de leur assiette, des entreprises à la charge publique.

En ce qui concerne l'Alcan, proportionnels à la dimension de ses activités et à sa puissance financière, les versements effectués à titre d'impôts divers sont très importants. Point n'est besoin de prendre en considération ceux qui vont aux gouvernements de la Province et du Canada. Ils ne profitent que très indirectement à la région. Par contre, le produit des taxes payées aux municipalités et aux Commissions scolaires régionales contribue directement au financement des services publics régionaux et au développement du système scolaire. Selon les renseignements par l'Alcan, de 1967 à 1971, les montants déboursés à titre de taxes municipales et scolaires se sont élevés respectivement à 9776064 dollars et à 18303141 dollars. C'est la municipalité, où, sont localisées les principales immobilisations de l'Alcan, qui bénéficie en priorité de cette manne. Ainsi, en 1971, Arvida recevait de la Compagnie plus d'un million de dollars de taxes. Il est peut-être intéressant de voir la ventilation du produit de ces taxes et leur évolution récente 119.

119 Voir le tableau 36. 
Tableau 36

Ville d'Arvida : relevé des taxes payées par la compagnie
Alcan pour les dix dernières années

$\underline{\text { Retour à la table des matières }}$

\begin{tabular}{|c|c|c|c|c|c|}
\hline Année & $\begin{array}{c}\text { Taxes foncières } \\
\text { générales }\end{array}$ & $\begin{array}{c}\text { Améliorations } \\
\text { locales }\end{array}$ & $\begin{array}{c}\text { Taxe } \\
\text { d’affaire }\end{array}$ & Taxe d'eau & Total \\
\hline 1972 & 1309441 & 20912 & 31100 & 119810 & 1481263 \\
\hline 1971 & 1113620 & 12651 & 28852 & 117411 & 1272534 \\
\hline 1970 & 995017 & 13056 & 300 & 113811 & 1122184 \\
\hline 1969 & 994939 & 15045 & 300 & 121010 & 1131294 \\
\hline 1968 & 1003590 & 13915 & 300 & 119810 & 1137615 \\
\hline 1967 & 866297 & 14490 & 300 & 101100 & 982187 \\
\hline 1966 & 804329 & 16280 & 300 & 96776 & 917685 \\
\hline 1965 & 805705 & 14523 & 300 & 97136 & 917664 \\
\hline 1964 & 812707 & 15562 & 300 & 93296 & 921865 \\
\hline 1963 & 706579 & 17262 & 300 & 94016 & 818157 \\
\hline
\end{tabular}

Source :Municipalité d’Arvida, 1972.

Toutes les données et analyses qui précèdent convergent vers une première conclusion qui apparaît évidente, à savoir que l'Alcan détient une place considérable dans l'économie du Saguenay-Lac-St-Jean. On peut même aller plus loin, en disant que la région est dans une dépendance si étroite à l'égard de la Compagnie qu'elle ne peut plus s'en passer aujourd'hui. Imagine-t-on en effet l'Alcan fermant ses usines et arrêtant ses activités au Saguenay-Lac-StJean. Les conséquences en seraient catastrophiques pour cette région. Mais, que les activités de l'Alcan soient désormais vitales pour l'économie saguenayenne ne signifie nullement qu'elles ont été un pôle de développement pour la région. Rien de ce qui précède, en effet, n'assure que l'Alcan puisse être considérée comme le solide maillon de la chaîne que formeraient les diverses entreprises industrielles régionales. L'analyse des effets indirects et induits de l'Alcan s'avère ainsi une démarche complémentaire indispensable pour la saisie de son impact sur l'économie du Saguenay_Lac-St-Jean. 


\section{Section 2 : \\ Les effets indirects et induits de l'Alcan}

Retour à la table des matières

De manière assez large, on peut définir les effets indirects d'une entreprise industrielle comme étant «la valeur ajoutée formée dans les autres entreprises d'une région, à l'occasion de la création ou du fonctionnement du complexe »120. Les mécanismes par lesquels ils se produisent relèvent en partie du phénomène de la multiplication et de l'accélération, connu en théorie économique comme étant à la base de la croissance. Le nouvel ensemble peut effectuer des achats dans la région, ajoutant sa demande à la demande globale. Il suscite ainsi un effort supplémentaire dans l'appareil régional de production, qui peut se traduire par l'élargissement progressif des activités anciennes ou l'émergence d'activités jusque-là inexistantes. Des investissements supplémentaires sont consentis, de nouveaux travailleurs embauchés, de sorte qu'il se forme un surcroît de revenus monétaires, susceptibles de déclencher à leur tour de nouveaux accroissements de capacités. Par ailleurs, le complexe distribue des revenus aux travailleurs de la région et constitue ainsi un support au développement du commerce régional. Si, de surcroît, la production du nouveau complexe entre comme produits intermédiaires dans la fabrication d'autres articles, il peut constituer un puissant facteur d'attraction à de nouvelles entreprises qui viendront s'installer dans son voisinage. De ce mouvement, il résulte un accroissement de la valeur ajoutée de l'ensemble des entreprises de la région. De manière plus systématique, on essaiera de l'analyser à travers les effets polarisateurs caractéristiques de l'Alcan.

120 F. Rosenfeld, op. cit., p. 78. 


\subsection{La polarisation technique}

$\underline{\text { Retour à la table des matières }}$

Les deux canaux par lesquels arrive l'industrialisation induite se confondent avec la polarisation technique, dont les deux aspects essentiels sont les effets de débouché ou effets de liaison en amont et les effets d'approvisionnement ou effets de liaison en aval. Suivant l'expression de J. Paelinck, ils constituent «les conditions nécessaires mais non suffisantes du développement régional polarisé » 121. L'industrie d'aluminium semble favoriser la naissance de tels effets. Certes, Chenery et Wanatabe, dressant une échelle des diverses industries sous le rapport de leurs effets en amont et en aval, ont établi que l'industrie des métaux non-ferreux vient en second rang, après celle de l'acier, pour ses effets en aval. Beaucoup moins importants, les effets en amont de cette industrie cumulent cependant un maximum de 61 points contre 81 pour celle de l'acier. Il est vrai que les calculs de ces auteurs ont été effectués sur la base de données enregistrées dans seulement trois pays : l'Italie, le Japon et les Etats-Unis 122. En dépit des présomptions favorables dégagées ci-dessus, seul l'examen de la situation particulière de l'économie saguenayenne à l'égard de l'Alcan permettra de juger. Certes, « il ne faut pas considérer a priori une implantation industrielle comme un pôle de croissance nécessairement efficient sous lequel se produisent des relations de polarisation effectives, techniques ou autres » 123.

\subsubsection{Les effets de liaison en amont de l'Alcan}

Quoique, de par sa nature, un complexe électro-métallurgique requiert une consommation intermédiaire importante, l'examen, même superficiel, de la structure industrielle du SaguenayLac-St-Jean révèle qu'une véritable industrialisation, à partir des fournisseurs des usines de l'Alcan, ne s'est pas produite. La probabilité de réalisation d'un tel événement était du reste très faible au départ. On sait en effet que la plupart des inputs-matières de l'industrie régionale d'aluminium

121 J. Paelinck, op. cit., ISEA,L-15, mars 1965, p. 36.

122 Cités par A. O. Hirschman, op. cit., pp. 125-126.

123 J. Paelinck, op.cit., p. 13. 
lui proviennent de l'extérieur. Certes, la transformation de la bauxite en alumine s'opère sur place dans les usines d'Arvida. Un important laboratoire de recherches a été créé dans cette ville, devenue « un centre important dans le domaine de la chimie inorganique » 124. Mais, si l'on ne tient pas compte de ces activités, essentiellement intégrées, du reste, à la production de l'aluminium primaire, le champ d'application de l'effet Léontief se trouve singulièrement restreint, coïncidant plus ou moins avec la mobilisation de la seule ressource régionale disponible : l'énergie hydroélectrique. Par ailleurs, les inputs en capitaux techniques, matériel et équipements, très spécialisés, ne pouvaient être fournis que par des entreprises localisées en des zones hautement industrialisées. Ce n'était pas le cas du Saguenay_Lac-St-Jean au moment de l'implantation de l'industrie d'aluminium. Or, le voisinage d'une clientèle réduite aux seules usines de l'Alcan ne constituait pas un motif assez puissant pour attirer de telles entreprises dans la région. Il ne restait que deux domaines pour le développement d'éventuels effets de liaison en amont : les ressources hydrauliques et les infrastructures de base.

\subsubsection{L'Alcan et l'énergie hydroélectrique au Saguenay-Lac-St-Jean}

« En posant que chaque région contient une ressource potentielle et que chaque période contient l'innovation qui fait de la ressource potentielle une ressource effective, chaque région est successivement animée par l'innovation qui la concerne. C'est une impulsion étrangère aux variables retenues dans le modèle régional qui suscite le développement et la croissance ». On ne saurait trouver mieux trouver que cette longue citation du Professeur Perroux 125 pour qualifier l'action exercée par l'Alcan sur la région du Saguenay. Il n'est pas nécessaire de s'attarder ici sur la considérable expansion enregistrée par la production hydroélectrique du Saguenay—Lac-St-Jean à partir de la dynamique impulsion de l'Alcan 126. Pour mémoire, on peut rappeler toutefois que de 1920 à 1928 la production régionale s'est multipliée par plus de huit, passant de 104000 chevauxvapeur à 850 000. Aujourd'hui, la capacité globale de production hydroélectrique régionale se situe autour de 3400000 chevaux-vapeur, dont plus de 92\% ont été produits et sont sous le contrôle de l'Alcan.

124 Alcan, L’Alcan au Québec, 1967.

125 F. Perroux, L’économie du XXe siècle, op. cit., p. 204.

126 Voir plus haut ; « Alcan entreprise régionale ». 
On doit certes souligner que les usines de la Compagnie consomment la majeure partie de l'énergie produite, 65 à 70\% en période d'activité normale, selon P.-Y. Pépin 127. Il n'en reste pas moins que l'Alcan reste un grand fournisseur de la région en énergie électrique, pour des fins industrielles aussi bien que domestiques. En ce sens, la présence de l'Alcan a entraîné la croissance de certaines branches industrielles, dont le type de production n'entretient apparemment aucune relation avec l'industrie d'aluminium. Tel semble être le cas de l'industrie papetière, pour n'en retenir que la plus importante. C'est dans la mouvance de l'industrie d'aluminium que celle du papier, second pilier de l'activité économique régionale, a pu opérer sa transformation et enregistrer sa propre expansion. Il est en effet révélateur, compte tenu de l'immensité des ressources forestières régionales, que la production papetière du Saguenay-LacSt-Jean, stagnant autour de 2000 tonnes en 1927, est passée à 1330000 tonnes en 1966. On ne sous-estime pas l'influence d'autres facteurs, comme l'extension de la demande mondiale, dans l'explication de ce phénomène. Mais, il n'est pas moins vrai que cette croissance aurait été impossible sans les énormes surplus d'énergie hydroélectriques dégagés par l'Alcan.

Dans cette perspective, on peut jusqu'à un certain point avancer que la présence dynamique de l'Alcan a entraîné des effets positifs sur la croissance économique régionale. Mais, en définitive, c'est à l'énergie hydroélectrique plus qu'à tout autre facteur qu'il convient d'attribuer le rôle moteur, dans la mesure où elle a suscité la création et le développement des deux industries majeures de la région : l'aluminium et le papier.

\subsubsection{L'Alcan et les infrastructures du Saguenay-Lac-St-Jean}

Si l'industrie d'aluminium n'a pratiquement pas pu entraîner d'authentiques et importantes complémentarités en amont, on ne peut pas en dire autant de l'influence qu'elle a exercée sur le développement des infrastructures régionales. Point n'est besoin d'insister ici sur l'importance primordiale que revêtent les investissements de base sur la croissance d'une économie, particulièrement au moment de son démarrage. En ce qui concerne le

127 P.-Y. Pépin, op. cit., p. 354. 
Saguenay_Lac-St-Jean, l'Alcan a été directement ou indirectement, l'un des principaux artisans des progrès enregistrés par la région dans ce domaine 128.

En effet, mis à part les investissements effectués en vue d'une exploitation intensive et rationnelle des ressources hydrauliques, la Compagnie a consacré d'importantes sommes à la mise en place ou à l'amélioration de certaines infrastructures de la région, notamment en matière de transport. À ce titre, on doit rappeler que l'Alcan a été le principal artisan des aménagements portuaires de Port-Alfred, reconnu aujourd'hui comme le principal port de la région et un nœud vital pour ses activités économiques. Il est incontestable que Port-Alfred constitue un puissant facteur d'attraction et de croissance pour les industries du Saguenay—LacSt-Jean, en particulier pour celles qui, comme l'industrie papetière, sont tributaires du marché extérieur pour l'écoulement de leur production.

La Compagnie, par ailleurs, a participé de manière directe à la mise en place du réseau des voies ferrées de la région. Aujourd'hui encore, deux filiales de l'Alcan, « Roberval \& Saguenay Limited » et «Alma-Jonquière », exploitent des lignes qui couvrent près de 70 kilomètres. Il n'est pas jusqu'aux installations de l'aérodrome militaire de Bagotville et à l'aéroport régional de cette ville, située à une dizaine de milles de la Cité de Chicoutimi, qui ne doivent être mis, même très indirectement, à l'actif de l'Alcan. Certes, compte tenu de l'importance stratégique de l'aluminium, il s'avérait une nécessité de créer dans le voisinage du complexe d'Arvida une base de défense. C'est peut-être dans la même perspective qu'il importe de comprendre la mise en place par le gouvernement fédéral, entre 1939 et 1944, des installations (trois pistes d'envol) de SaintHonoré, petite localité à quelques milles au nord d'Arvida. Il est difficile de mesurer les économiques de ces infrastructures. Mais, l'aéroport régional de Bagotville, notamment, favorise l'activité économique régionale, dans la mesure où il permet le déplacement rapide des hommes d'affaires de la région. En 1966, environ 40000 personnes avaient emprunté ce moyen commode de transport.

128 À ce propos, voir plus haut, «L’Alcan et le problème des transports régionaux ». 
À côté des effets de l'implantation de l'Alcan sur le système régional de transport, on signalera en outre la fondation en 1926 de la ville d'Arvida. Il est bien connu que «partout la ville est le vecteur de la vie moderne.., et en tant que centre de consommation, elle peut stimuler les productions agricoles comme les productions industrielles » 129. Certes, à son origine, le caractère fermé de la nouvelle ville, propriété de l'Alcan, dont il fallait obtenir l'autorisation pour y demeurer, constitua un élément certain de freinage à sa croissance. Mais, très rapidement, Arvida est devenue ville ouverte, avec un Conseil d'Administration indépendant de la Compagnie. À partir de 1954, les ouvriers de l'usine d'aluminium résidant à Arvida, jusque-là locataires pour la plupart des maisons de l'Alcan, purent devenir plus facilement propriétaires de leurs logements. Il n'en reste pas moins cependant que l'Alcan, encore aujourd'hui, avec $10 \%$ des propriétés résidentielles et commerciales, demeure le principal propriétaire à Arvida. D'un autre côté, malgré sa population estimée aujourd'hui à 20000 habitants environ, cette ville reste « une usine et un habitat pour la main-d'œuvre au service de l'aluminium » 130.

Qu'il ait pu résulter de cette création d'Arvida « un fait culturel facilitant l'effet de signification » ou encore « se former de nouvelles capacités spécifiques et une aptitude globale au développement, s'exprimant dans la population par une manière de vivre et une mentalité favorables à la création et au progrès » 131 semble être une hypothèse valable qui sera examinée dans le cadre de l'étude des effets psychologiques engendrés par l'Alcan. Auparavant, il convient de se pencher sur le deuxième aspect de la polarisation technique.

\subsubsection{Les effets de liaison en aval de l'Alcan}

L'aluminium, comme on sait, entre dans le processus de fabrication d'un nombre illimité de produits finis. Ainsi, le Saguenay_Lac-St-Jean qui, par l'intermédiaire de l'Alcan, produit annuellement plus de 400000 tonnes d'aluminium, et dispose en outre d'importantes réserves en énergie hydroélectrique, avait apparemment toutes les chances de se doter d'une puissante industrie en aval de l'aluminium. Toutefois, une remarque de G. Bell invite à la prudence, qui souligne que

129 G. Bell, op. cit., p. 165.

130 P.-Y. Pépin, op. cit., p.159.

131 F. Perroux, « La firme motrice dan la région et la région motrice », Cahiers de l'ISEA, op. cit., p. 43. 
« comme matière première, l'aluminium ne présente qu'un intérêt restreint pour un pays en voie de développement, qui n'a aucun débouché à lui offrir et n'a pas les moyens de lui en créer »132. Le degré de développement de la région lui permettait-il de tirer pleinement parti de l'aluminium offert par l'Alcan?

La simple observation de l'activité économique au Saguenay-Lac-St-Jean révèle « l'absence d'importantes usines d'aval de produits finis et semi-finis » de l'aluminium 133. Cependant, à l'ombre de la puissante machine industrielle de l'Alcan se sont édifiées une série de petites et moyennes entreprises. Un inventaire de ces dernières servira de point de départ à l'analyse des effets de polarisation en aval de l'Alcan.

Cette imposante liste d'entreprises, nées sous l'influence directe de l'industrie d'aluminium, pourrait laisser croire de prime abord que l'Alcan a entraîné à sa suite d'importantes activités au Saguenay-Lac-St-Jean, complémentaires en aval de l'aluminium. Cette impression se justifierait d'autant plus que l'on sait l'importance de la dimension des flux, réels et monétaires, dont cette Société est à l'origine. Mais, en matière de développement polarisé, le vrai problème consiste à savoir si les flux d'offre de produits des entreprises de l'Alcan sont d'une intensité plus grande à l'intérieur de la région qu'à l'extérieur. Considéré sous cet angle, le problème se révèle particulièrement simple. Il est un fait, du reste déjà souligné, que la presque totalité, plus de 95\%, de la production d'aluminium de l'Alcan, au Saguenay-Lac-St-Jean, est écoulée à l'extérieur de la région. Ainsi, l'industrie d'aluminium, ne trouvant pas de débouché réel auprès des entreprises régionales, n'entretient, à la vérité, que des relations marginales avec l'économie de la région, relativement du moins à la dimension de ses propres flux. Qu'en est-il alors de cette multitude de petites entreprises « consommatrices d'aluminium »?

132 G. Bell, op. cit., p. 213.

133 P.-Y. Pépin, op. cit., p. 213. 
Tableau 37

Saguenay-Lac-St-Jean : industries de services et industries utilisant l'aluminium

$\underline{\text { Retour à la table des matières }}$

\begin{tabular}{|c|c|}
\hline \multicolumn{2}{|l|}{ Industries de services } \\
\hline 1. Atelier mécanique & 3. Fabrication - divers \\
\hline - $\quad$ Atelier mécanique d’Alma Enr. & - Alma Mix \& Supply Co. Ltd \\
\hline - $\quad$ Arvida Welding \& Repair Shop Enr. & - $\quad$ Arvida mix \$ Supply Ltd \\
\hline - $\quad$ Ludger Harvey \& Fils Ltée & - Béton du Saguenay \\
\hline - $\quad$ Lar Machinery & - Dunbrick Ltée \\
\hline - Kenogami Welding \& Mechanic Reg. & - $\quad$ Grenon \& Fils Ltée \\
\hline 2. Entrepreneurs & - $\quad$ Industries Couture Ltée \\
\hline - J. Achard Ltée & - $\quad$ Pic Construction Co. Ltd \\
\hline - $\quad$ Alma Construction Ltée & - $\quad$ Potvin \& Bouchard Inc. \\
\hline - C.A.B. Construction & - $\quad$ Saguenay Industries Ltd \\
\hline - $\quad$ Demeules \& Duchesne Ltée & 4. Services et Fournitures \\
\hline - $\quad$ Laval Fortin Ltée & - R. Blackburn Ltée \\
\hline - $\quad$ Grimard Électrique Enr. & - $\quad$ P.S. Desautels Inc. \\
\hline - $\quad$ Isolation Régionale Enr. & - $\quad$ A.E. Gauthier Ltée \\
\hline - $\quad$ Le Chauffage Moderne d’Arvida & - $\quad$ M. \& M. Propane Gas Co. Ltd \\
\hline - J.-Euclide Perron Ltée & - $\quad$ Saguenay Oxygen Ltd \\
\hline \multicolumn{2}{|l|}{ Industries consommatrices d'aluminium } \\
\hline 1. Consommatrices d'aluminium & $\begin{array}{l}\text { 3. Acheteurs de fils électriques ou } \\
\text { distributeurs de conducteurs Alcoconduit }\end{array}$ \\
\hline - $\quad$ Couture \& Bergeron, Chicoutimi & - Hydro-Québec, Chicoutimi \\
\hline - $\quad$ Chauffage Moderne, Arvida & - $\quad$ Canadian Westinghouse, Chicoutimi \\
\hline - $\quad$ Lustro-Métal, Jonquière & - $\quad$ Northern Electric, Chicoutimi \\
\hline - $\quad$ Lar Machinery, Métabetchouan & - $\quad$ Vandry Inc., Chicoutimi \\
\hline - $\quad$ Idustrie Couture, Chicoutimi & - $\quad$ J. Achard Ltée, Jonquière \\
\hline - $\quad$ Jalbert Ltée, Chicoutimi & \\
\hline - Ludger Harvey \& Fils, Jonquière & \\
\hline \multicolumn{2}{|l|}{ 2. Distributeurs du Revêtement Alcan } \\
\hline - G. Desbiens Aluminium Ltée, Chicoutimi & \\
\hline - $\quad$ Desbiens \& Bouchard, Bagotville & \\
\hline - $\quad$ Produits de Construction d’aluminium, Alma & \\
\hline - $\quad$ Lac-St-Jean Aluminium Ltée & \\
\hline
\end{tabular}

Source : P.-Y. Pépin, op. cit., pp. 213-214. 
Sur ce sujet, l'analyse se complique, faute de documents comptables qui permettraient de mesurer, de manière plus ou moins précise, l'importance financière et industrielle des entreprises dépendantes de l'Alcan. Il est du reste révélateur que la Compagnie, généralement bien disposée à fournir des renseignements sur ses activités, ne publie aucune statistique relative à la quantité et à la valeur de l'aluminium vendu dans la région. Plus qu'à la crainte d'éventuels concurrents, c'est à la faiblesse, sinon à l'inexistence d'un marché régional d'aluminium qu'il convient d'attribuer ces lacunes. On en arrive ainsi à conclure que cette multitude de petites entreprises ne pèsent pas lourd dans l'économie régionale aussi bien que dans le chiffre d'affaires de la Société. Cette conclusion peut être corroborée par l'examen de la situation de l'industrie régionale de produits métalliques relativement à l'ensemble du secteur manufacturier.

Tableau No 38

Région : industrie des produits métalliques ; statistiques et poids relatif, 1965

$\underline{\text { Retour à la table des matières }}$

\begin{tabular}{|l|c|c|c|c|}
\hline Type d'entreprise & Emploi & Salaires et gages & Valeur ajoutée & Expéditions \\
\hline Architecture et ornementation & 48 & $178400 \$$ & $392500 \$$ & $668700 \$$ \\
\hline Quincaillerie, coutellerie, outillage & 1 & $3500 \%$ & $28700 \$$ & $38500 \$$ \\
\hline Pièces mécaniques & 103 & $461300 \$$ & $499100 \$$ & $1350800 \$$ \\
\hline Produits métalliques & 89 & $411200 \$$ & $150200 \$$ & $626900 \$$ \\
\hline Total industrie & 241 & $1054400 \$$ & $1070500 \$$ & $2684900 \$$ \\
\hline Pourcentage du secteur manufacturier & 1,7 & 1,3 & 0,4 & 0,6 \\
\hline
\end{tabular}

Source : Tiré du tableau numéro 4, dans Mission de planification du Saguenay_Lac-St-Jean : Situation actuelle et évolution récente de l'industrie manufacturière, miméo., août 1969, p. 31.

L'examen du tableau qui précède révèle que l'industrie métallique est responsable seulement de 0,4\% de la valeur ajoutée et de 0,6\% de la valeur des expéditions de l'ensemble du secteur manufacturier de la région. On peut également faire observer que cette branche fournit un emploi global relativement faible, représentant moins de $2 \%$ de la main-d'œuvre travaillant dans le secteur 
manufacturier. Toutes ces données concourent à démontrer le peu d'importance de l'industrie des produits métalliques dans l'activité économique du Saguenay-Lac-St-Jean. Il est donc vrai que l'industrialisation en aval de l'aluminium a été faible.

Certes, on a souligné que l'Alcan possède à Arvida des usines de laminage de l'aluminium, ainsi qu'un important secteur de fabrication de fils-machine, utilisés pour le transport de courant à haute tension. Le type d'investissements effectués par la Compagnie au cours de ces dernières années tendrait également à indiquer que l'activité de transformation de l'aluminium en produits finis pourrait prendre de l'ampleur dans un proche avenir. Mais, force est de modérer les espoirs qu'on serait en droit de formuler dans ce domaine. On ne peut que souligner, en ce sens, l'échec d'une tentative relativement lointaine de l'Alcan. En 1945 déjà, un organisme de recherche et de publicité « l'Association d'Expansion Industrielle du Saguenay », avait été fondée par l'Alcan, dont le but était d'attirer dans la région de nouvelles entreprises consommatrices d'aluminium et d'énergie hydroélectrique. Des considérations de «good-will » propre n'étaient sans doute pas étrangères aux efforts déployés par l'Alcan. Mais, le moins qu'on puisse dire est que les résultats obtenus sont plutôt décevants à coté de ceux qu'on avait escomptés, compte tenu des observations qu'on a faites plus haut relativement à l'industrialisation entraînée par l'aluminium. Les raisons de ce phénomène, apparemment paradoxal, seront analysées au chapitre suivant. Toutefois, on peut déjà souligner que l'étroitesse du marché régional, ajoutée à une mentalité plus commerçante qu'industrielle, y a joué un rôle de premier plan. Cela s'avère d'autant plus vrai que la présence et la participation de l'Alcan ont permis la création et la croissance de certaines activités importantes, comme la "Simonds Abrasive Company» et «Les Siliciums de Chicoutimi ».

Quoiqu'il en soit des effets en amont et en aval de l'industrie de l'aluminium, entraînée ellemême par l'énergie hydroélectrique, on peut admettre jusqu'à un certain point que l'avènement de l'Alcan au Saguenay_Lac-St-Jean y a suscité une mutation structurelle. Il est à souligner en effet que l'activité industrielle régionale ne comportait auparavant qu'une seule branche vraiment importante: l'industrie papetière, qui avait relayé les scieries. Par ailleurs, la faiblesse de la polarisation technique de l'Alcan, qui ne s'est pas étendue, jusqu'à présent, au-delà du couplage 
« énergie - hydroélectrique - aluminium », n'a pas empêché de s'exercer d'autres formes de polarisation, dont l'influence sur la croissance d'une région est tout aussi importante. Ces autres aspects de l'action de l'Alcan seront examinés dans les paragraphes qui vont suivre.

\subsection{Polarisation financière et polarisation par les revenus}

$\underline{\text { Retour à la table des matières }}$

Dans la deuxième partie de cette étude 134, on a analysé le caractère plurinational de l'Alcan et les éléments de sa puissance financière. Il est évident que le financement des entreprises régionales de la Compagnie, dont le siège social est à Montréal, s'est effectué à partir de l'extérieur. D'autre part, compte tenu de la faiblesse de l'industrialisation entrânée par l'Alcan dans le Saguenay_Lac-St-Jean, on peut dire que la Société n'a pas été à l'origine d'un drainage de capitaux financiers. Aucune analyse des sources de financement des activités industrielles de la région n'a encore été entreprise, de sorte qu'il est plus malaisé de mesurer ou même de définir les effets de polarisation financière qu'a pu avoir l'Alcan sur l'économie du Saguenay—Lac-St-Jean.

On sait cependant que les sommes engagées par la Société dans ses entreprises de la région sont relativement énormes. Considéré sous cet angle, il est incontestable que l'Alcan a suscité des effets financiers importants pour la région. De même, par ses prises de participation dans certaines entreprises, comme les "Siliciums de Chicoutimi », dont le coût d'établissement, en 1966, n'a pas été moins de 3,5 millions de dollars, l'Alcan contribue à la croissance régionale. Toutefois, c'est dans le domaine de la création et la distribution de revenus que la Société exerce le maximum d'effets polarisateurs.

On a déjà signalé le rôle majeur joué par l'Alcan dans le domaine de la création des emplois. Sur 14127 employés du secteur industriel, recensés dans la région en 1965, plus de 6800 étaient au service de l'industrie d'aluminium, soit environ 48\%. Des compilations plus récentes, (janvier 
1968), selon P.-Y. Pépin, montraient que le personnel global travaillant dans les entreprises de l'Alcan représentait plus de 10\% du volume régional de main-d'œuvre, soit exactement 8874 personnes sur un total de 87 000, dont 15000 en chômage 135.

Or, à cause de la jeunesse de sa population, le Saguenay-Lac-St-Jean possède l'un des taux de dépendance les plus élevés de la Province du Québec. Le taux de dépendance est ici défini comme étant le pourcentage de la population que font vivre les travailleurs. Sur la base de cet indice, main-d'œuvre / population / \%, le taux de dépendance, au Saguenay—Lac-St-Jean, serait égal à 3,5 environ contre seulement 2,9 pour l'ensemble du Québec. Ainsi, chaque employé de la région fait vivre plus de trois personnes en moyenne. Une analyse détaillée de cet indice montrerait d'ailleurs qu'il est plus important pour le personnel de l'Alcan, dont le niveau moyen de salaires est plus élevé, comparativement à celui des autres branches d'activités de la région. S'il est vrai que, dans l'état actuel des structures de l'économie saguenayenne, à un emploi du secteur secondaire correspondent en moyenne 2,3 emplois du tertiaire 136, on peut estimer à 3 l'effet multiplicateur de l'Alcan. Les revenus distribués par les entreprises de l'Alcan seraient ainsi à l'origine d'un intense processus multiplicateur, dont le champ d'application privilégié a été le secteur des services, en particulier les activités commerciales. Or, s'il est vrai encore que le développement de la grande industrie, dont le taux de progression est généralement plus élevé que celui des autres secteurs, est le facteur-clé de la croissance, i1 est évident que l'Alcan a joué un rôle majeur au SaguenayLac-St-Jean. De toute manière, il ne reste pas moins important pour une région de disposer d'un secteur dynamique formé de petites et moyennes entreprises. On sait en effet que «ces petites industries, relativement aux industries modernes de transformation, ont des chances supérieures de créer des possibilités d'emploi » 137.

En ce qui concerne l'industrie saguenayenne d'aluminium, on a montré ailleurs son rythme de progression, particulièrement rapide, dû sans doute à l'expansion considérable de la demande mondiale, consécutivement à la seconde guerre mondiale. Que la croissance autonome de cette

135 P.-Y. Pépin, op. cit., p. 212.

136 Selon le Bureau de Recherche Économique, le rapport était en 1965 de 38,5 \% pour l'ensemble du Québec.

137 BIT, «Les objectifs d'emploi dans le développement économique », dans Études et Documents, Nouvelles Séries, no 62, Genève, 1961, p. 5. 
industrie ait entraîné une modification radicale du climat économique du Saguenay-Lac-St-Jean et une mutation profonde de la structure de l'économie de cette région ne fait aucun doute. Un aspect non négligeable de ce phénomène réside dans l'accroissement de la demande régionale, manifesté notamment par l'augmentation des dépenses de consommation. Ce n'est pas le lieu ici de discuter des mécanismes par lesquels s'élabore la croissance économique. On doit néanmoins rappeler qu'à son origine se place le progrès technique, vecteur de l'accroissement de l'offre de production et de la demande. Comme le souligne G. Lebel, le processus complexe de la croissance « résulte d'une interaction entre l'augmentation de la demande induite par une augmentation de l'offre et l'augmentation de l'offre suscitée par la volonté de répondre à l'augmentation de la demande » 138. C'est le processus qui semble s'être déroulé dans le Saguenay—Lac St-Jean.

À partir de l'implantation du complexe d'aluminium d'Arvida, on y a assisté à un afflux de main-d'œuvre provenant de l'extérieur. À la main-d'œuvre régionale ainsi accrue sont distribués des salaires importants, grossissant d'autant la demande de la région en biens de consommation et en services. On peut penser que, dans un premier temps, l'appareil régional de production a été dans l'impossibilité de répondre à cet accroissement brusque de la demande et que le grossissement du circuit monétaire a entraîné une augmentation des importations et une certaine hausse des prix régionaux.

On ne dispose pas de données sur la structure de la demande et le mouvement des prix au Saguenay_Lac-St-Jean au moment de l'implantation de l'Alcan, en 1925-26. Mais, suivant une loi économique généralement vérifiée, «à de faibles niveaux de revenus correspond un pourcentage élevé de ces revenus (tant marginal que moyen) consacré à la nourriture, cette part tendant à décroître à mesure que s'accroît le revenu, au profit de produits manufacturés, de biens durables et de services » 139. Or, vers 1943, le salaire moyen d'un ouvrier d'Arvida s'élevait à 1 660 dollars, contre seulement 1424 dollars pour la Province du Québec et 1525 dollars pour le Canada. On sait d'autre part que c'est l'année où la moyenne de l'embauche a été la plus élevée dans les entreprises régionales de l'Alcan. Mais, si incontestablement cet afflux de salaires déclencha 
une vague de progrès dans l'agriculture et dans l'industrie des produits alimentaires, il reste également vrai que la région dut continuer à faire appel à l'extérieur pour couvrir l'ensemble de ses besoins 140 .

Aujourd'hui, la part du revenu consacrée aux dépenses pour la nourriture atteint, au Québec, 25\% en moyenne des dépenses totales. Si l'on se rappelle qu'en 1967 les revenus versés aux 9000 employés des entreprises saguenayennes de l'Alcan totalisaient 77400000 dollars, dont plus de 57 millions au personnel des deux alumineries d'Arvida et d'Isle-Maligne, on se rend aisément compte de limportance du processus multiplicateur qui peut être créé. Pourtant, la région reste encore déficitaire pour un nombre de produits alimentaires, ainsi qu'on peut le voir au tableau suivant .

Tableaux 39

Production et consommation de viandes en livres, région 1965

$\underline{\text { Retour à la table des matières }}$

\begin{tabular}{|l|c|c|c|}
\hline \multicolumn{1}{|c|}{ Produits } & Production & Consommation & Solde \\
\hline Bouf & 5525000 & 22193400 & -16668400 \\
\hline Porc & 7800000 & 13874400 & -6074400 \\
\hline Veau & 2800000 & 2256000 & +544000 \\
\hline Mouton & 210000 & Quasi nulle & +200000 \\
\hline Volaille & 6000000 & 9729000 & -3729000 \\
\hline
\end{tabular}

Source : P.-Y. Pépin, op. cit., p. 317.

Ce déficit persistant de la région pour la couverture de nombre de ses besoins en produits alimentaires tendrait à démontrer que l'agriculture, en dépit de certaines mutations de structure, n'a pas su bénéficier pleinement des effets d'entraînement apportés par les salaires additifs versés par les entreprises de l'Alcan. Il est certes vrai que l'agriculture du Saguenay-Lac-StJean est fortement spécialisée et que l'ensemble des activités agricoles convergent vers la production de produits laitiers, notamment du fromage. Plus de $75 \%$ des fermes régionales sont considérées comme des exploitations laitières et 96\% de la superficie du domaine des grandes 
cultures est consacrée aux fourrages et grains pour les bovins 141. Aussi, de 1951 à 1967, voit-on passer la production régionale de fromage de 824000 livres à 12196000 livres. Il semble donc que dans le domaine agricole, comme dans le domaine industriel, la région préfère produire davantage les biens pour lesquels elle se sent la mieux dotée, quitte à s'en remettre à l'extérieur pour l'obtention de ceux qu'elle ne produit pas en quantités suffisantes. Il n'est dès lors pas étonnant qu'en 1966, sur 259632000 dollars de biens et services importés par la région, 83572 000 étaient consacrés à l'achat de produits alimentaires (y compris les produits de laiterie et de pâtisserie). On notera que pour la même année, les exportations s'élevaient à 241626000 dollars 142.

Des observations qui précèdent, il est aisé de tirer trois conclusions qui semblent reliées étroitement entre elles. Tout d'abord, l'Alcan, en dépit de son dynamisme propre, n'a exercé qu'une faible polarisation à l'égard de l'industrie régionale. Deuxièmement, s'il est incontestable que, par la masse des revenus distribués dans le Saguenay-Lac-St-Jean, la Compagnie a vigoureusement contribué à l'expansion de certaines activités de la région, il n'en reste pas moins vrai qu'une importante partie du processus multiplicateur se joue à l'extérieur. À la vérité, l'effet Capet, particulièrement élevé, s'inscrit dans la réalité des structures économiques régionales et se traduit par l'importance des importations. Enfin, comme corollaire des deux premières conclusions, on peut dire que c'est au niveau du secteur tertiaire que les revenus créés et distribués par l'Alcan devaient entraîner le plus d'effets.

Le phénomène de la singulière expansion du secteur tertiaire, au sein de l'économie du Saguenay_Lac-St-Jean, a été analysé ailleurs dans la présente étude 143. Le rappel de trois exemples particulièrement frappants suffira ici au propos. Sur la base de 100 en 1951, l'indice de la valeur ajoutée brute en dollars courants est passé, en 1966, à 682 pour le commerce de gros, à 591 pour les services et à 916 pour l'Administration publique 144. Cette évolution, comme on l'a souligné, s'est

141 P.-Y. Pépin, op. cit., p. 307.

142 CÉR du Saguenay-Lac-St-Jean : Étude économique et financière de la région du Saguenay-Lac-St-Jean, Comité des Finances, Jonquière, 1967-1968.

143 Voir plus haut, L’Évolution du secteur tertiaire.

144 Données extraites du tableau 11, dans A. Saicans, op. cit., p. 41. 
traduite par l'accroissement du poids relatif de ce secteur dans la structure de l'économie régionale. Si l'on en croit G. Lebel, le même phénomène semble s'être produit au niveau de l'ensemble du Québec. «Ainsi, au Québec, en 1946, l'emploi dans les services occupait environ 596000 travailleurs, soit à peu près 45\% de la population active ; aujourd'hui, il occupe 1200000 personnes, soit $58 \% » 145$.

En ce qui concerne l'évolution du secteur tertiaire au Saguenay_Lac-St-Jean, la part à mettre à l'actif de l'Alcan est importante. Considérant en effet qu'un employé de la région fait vivre en moyenne 3,5 personnes et qu'un emploi du secteur industriel est susceptible de créer 2,3 emplois, on est en mesure de conclure que les 7000 employés des industries d'aluminium de la région créent indirectement du travail à environ 14000 personnes, dont la majeure partie se trouveraient dans l'une ou l'autre des branches du secteur tertiaire. C'est ainsi par la polarisation des revenus que les entreprises de l'Alcan ont exercé, le plus d'influence sur l'économie du Saguenay_Lac-St-Jean. Reste à déterminer si les modifications suscitées par l'Alcan au niveau des structures économiques proprement dites ont été accompagnées de mutation des mentalités (polarisation psychologique) et de changements dans l'environnement de la région (polarisation spatiale).

\subsection{La polarisation psychologique}

$\underline{\text { Retour à la table des matières }}$

Fondamentalement, la polarisation psychologique que peut exercer la grande firme sur son environnement comporte deux aspects qui semblent étroitement reliés entre eux. D'une part, le nouveau complexe industriel incite les autres entreprises de la région à consacrer une partie de plus en plus importante de leurs ressources à se moderniser. La force de l'exemple de la grande firme, soucieuse d'efficacité dans la gestion et de productivité par l'introduction dans le processus de leur activité de production de l'acquis du progrès technique, peut devenir en quelque sorte contagieuse. Les petites et moyennes entreprises les plus dynamiques de la région guideront,

145 G. Lebel, op. cit., p. 201. 
de façon consciente ou inconsciente, leur comportement sur celui de la grande firme. D'autre part, du côté de la demande se jouera un phénomène analogue. L'aspect de nouveauté que représente la grande firme et le début de prospérité qu'elle apporte, par un surcroît de revenus à la région, s'accompagneront d'une certaine euphorie visible dans le comportement des agents économiques régionaux. La soif de produits nouveaux de consommation se fait contraignante, entraînant l'accroissement des dépenses d'achats. Il n'est même pas rare que, poussés par le double " effet de démonstration » et "d'imitation », les sujets économiques comblent par le crédit le déficit de leur budget. Évidemment, ces deux éléments de la polarisation psychologique se produiront avec d'autant plus de force qu'ils pourront s'appuyer sur les relais d'un solide réseau de communications, diffusant rapidement les informations d'un bout à l'autre de la région. Dans cette optique, on peut souligner avec le Professeur Perroux que «l'apparition d'une ou de plusieurs industries change l'atmosphère d'une époque, crée un climat favorable à la croissance et au progrès... La nouveauté élargit l'horizon économique ; réussie, elle a valeur d'exemple et suscite des imitations elles-mêmes créatrices » 146.

Les séquences décrites ci-dessus de la polarisation psychologique sont d'autant plus intéressantes qu'elles sont les simples manifestations de surface du phénomène de la mutation des structures mentales des populations de la région. L'impulsion de la firme nouvelle entraîne la modification des habitudes mentales, du mode de vie et même des attitudes face à l'existence. Progressivement, de nouvelles valeurs peuvent naître: dans le meilleur des cas, le fatalisme traditionnel fait place au goût du risque et à l'optimisme ; à l'éternel résigné succède un homme nouveau, avide de produire et de consommer. Ces effets souhaitables de la firme nouvelle comportent cependant leur revers : ce sont les risques de frustration, porteuse de troubles sociaux, qu'entraînent l'optimisme impuissant et les désirs inassouvis. À coup sûr, l'implantation et le développement des entreprises de l'Alcan, au Saguenay—Lac-St-Jean, ont entraîné des phénomènes analogues à ceux décrits plus haut. Par l'aménagement des châteaux d'eau de la région, la construction de la ville d'Arvida, l'édification dans cette dernière localité de la plus importante usine d'aluminium du monde, l'Alcan a véritablement inauguré un « climat » nouveau dans la région du Saguenay-Lac-St-Jean. Cela n'a certes pas été de l'envergure de la «rush» vers l'or ou de la

146 F. Perroux, L’économie du XXe siècle, op. cit., p. 148. 
découverte du Nouveau Monde. Mais, au moins pendant la période de l'implantation, la région a été un pôle d'attraction pour la main-d'œuvre extérieure. Il n'est pas exagéré de comparer les chantiers ouverts dans la région vers 1925-26 par l'Alcan à la découverte de ces « nouvelles frontières » qui ouvrent presque toujours des périodes de croissance durables.

Cependant, on doit faire observer que la force contagieuse de l'Alcan ne s'est pas exercée de manière particulièrement frappante sur les autres entreprises de la région. Un fait déjà souligné semble à ce propos révélateur : c'est la survivance au sein de l'économie régionale d'une multitude de petites manufactures à caractère artisanal. On ne peut certes pas soutenir qu'il y a une grande diffusion, dans les entreprises du Saguenay_Lac-St-Jean, des techniques modernes de gestion ou de l'esprit de recherche appliquée, quand on sait que plus de 75\% des établissements manufacturiers emploient un personnel variant entre un et quinze employés, n'assurent à peine que 2,5\% de la valeur ajoutée de l'ensemble du secteur industriel et 4\% de la valeur des expéditions. L'application systématique des méthodes modernes du «management » et l'appel au progrès technique semblent être restés le privilège de l'Alcan et de l'industrie papetière et de quelques autres établissements, dans l'industrie des produits alimentaires. On peut même se demander dans quelle mesure la puissante machine industrielle de l'Alcan n'a pas contribué à créer un certain « complexe » chez les petites et moyennes entreprises de la région, sensibles à l'exemple, mais incapables d'imiter, faute de ressources.

Par contre, il est incontestable que la présence au sein de l'économie régionale du plus vaste complexe d'aluminium du monde a entraîné un bouleversement des habitudes et des modes de vie de la population. Visibles au premier coup d'œil, ces effets se manifestent dans la fierté, mêlée de snobisme, des habitants, traditionnellement simples, de cette région. Les effets de démonstration et d'imitation se sont conjugués pour faire de ces « nouveaux riches » les consommateurs les plus assidus des derniers gadgets apportés par le progrès technique. La richesse étalée des bungalows, le luxe des nombreuses automobiles qui circulent, le confort intérieur des maisons et la multiplication des magasins de grande surface sont autant d'indices de la prospérité régionale. 
Est-ce à dire que cette « atmosphère » nouvelle a été sans failles ? On a avancé plus haut l'hypothèse d'un complexe d'infériorité qu'une Société aussi puissante que l'Alcan a pu créer chez les entrepreneurs locaux. Il est aussi vrai que les bienfaits de cette ère nouvelle inaugurée par l'Alcan n'ont pas pu être étendus à toutes les couches de la population régionale. De toute manière, ceux qui, comme les nombreux chômeurs du Saguenay-Lac-St-Jean, n'y participent pas, sont informés par les mass-media et voient vivre les plus fortunés. Il se crée ainsi des sentiments de frustration, qui peuvent déboucher sur des troubles très graves, mais qui ont surtout entraîné, jusqu'à présent, un certain exode vers d'autres régions. Cela amène à considérer le phénomène des effets spatiaux de l'Alcan sur le Saguenay_Lac-St-Jean.

\subsection{La polarisation spatiale}

\section{$\underline{\text { Retour à la table des matières }}$}

Selon le mot du Professeur Perroux, « il n'y a pas de croissance ni de développement sans concentration et expansion»147. De manière encore plus large, on pourrait dire que l'industrialisation entraîne la formation de véritables agglomérations, où se regroupent tout naturellement les hommes avec leurs ressources. Il naît ainsi un processus d'urbanisation à l'origine duquel peut se trouver l'action d'une grande firme, dispensatrice de salaires élevés et créatrice d'économies externes importantes. L'attraction qu'exerce la grande firme sur les facteurs de production, main-d'œuvre et capital, peut devenir déterminante pour la transformation du milieu d'implantation.

Tous ces phénomènes entrent dans le cadre de ce qu'on convient d'appeler « la polarisation spatiale » de la grande firme. Point de doute que ce mouvement peut s'accompagner de certains aspects négatifs. Ce serait même inéluctable, dans la mesure où tout déplacement se fait d'un point vers un autre. L'attraction exercée par un point sur les facteurs de production se fera ainsi au détriment d'autres points : la concentration et l'expansion du milieu d'implantation de la grande

147 F. Perroux, op. cit., p. 265. 
firme entraîne, au moins dans un premier temps, vide et dépression ailleurs. Cette analyse s'applique-t-elle à l'action exercée par l'Alcan sur le Saguenay_Lac-St-Jean ?

Il n'est pas nécessaire de reprendre ici les longs développements qui ont été consacrés à l'historique de l'arrivée et de l'implantation de l'Alcan dans la région. On doit cependant souligner, dans le cadre de ce propos, que l'Alcan a joué un rôle de premier plan dans la transformation de l’œkoumène du Saguenay_Lac-St-Jean. À ce sujet, la concomitance entre les grandes étapes de l'urbanisation de la région et la mobilisation d'une ressource latente est particulièrement remarquable. Ainsi, comme le souligne L.-M. Bouchard, «tous les stades de l'urbanisation du Saguenay se sont appuyés sur l'exploitation d'une ressource naturelle. Et sans vouloir minimiser, dans ce sens, ce qu'a été le rôle des papeteries régionales, on peut dire que l'exploitation de l'hydroélectricité, avec pour conséquence l'implantation de l'aluminium, a été le facteur déterminant de cette évolution » 148. Cette assertion sera examinée à travers l'analyse de deux éléments majeurs de l'évolution du Saguenay_Lac-St-Jean : l'accroissement démographique général et la croissance urbaine.

\subsubsection{L'évolution démographique}

On a déjà souligné dans la présente étude le rôle éminent joué par l'hydroélectricité dans le mouvement d'industrialisation de la région. Il est un fait que l'équipement de la grande chute d'IsleMaligne a donné lieu à une véritable «floraison industrielle », illustrée principalement par l'implantation de l'industrie d'aluminium et l'expansion des activités papetières régionales. Sans vouloir minimiser le rôle de la natalité, traditionnellement vigoureuse dans cette région, on peut dire que l'industrialisation ainsi amorcée, par l'afflux de main-d'œuvre qu'elle a entraîné, a exercé une action majeure sur le mouvement général de la population régionale. Il est significatif que les gains les plus importants de la région, en chiffres de population, se placent pendant la période dintense industrialisation.

Entre 1921 et 1931, la population du Saguenay_Lac-St-Jean est passée de 73117 à 105977 habitants, soit un accroissement absolu de 32860 habitants. Comparée à l'évolution démographique

148 L.-M. Bouchard, La conurbation du Saguenay (Québec), thèse de doctorat, Université de Strasbourg, 1971. 
des deux décennies précédentes, soient les gains successifs de 13458 habitants entre 1900 et 1911 et de 22631 entre 1911 et 1921, celle-là s'avère relativement forte. Mais, c'est au cours de la deuxième vague d'industrialisation, dont l'apogée se situe pendant la seconde guerre mondiale, que la région aura enregistré le taux d'expansion démographique le plus élevé. En effet, entre 1931 et 1943, la population régionale est passée de 105977 à 174300 habitants, soit un gain absolu de 68323 unités et un taux d'accroissement de 64,5\%. Aujourd'hui, la population régionale, estimée à 300 000 habitants environ, représente approximativement 4,5\% de l'ensemble de la population de la Province du Québec. Ce rapport a évolué de manière favorable, quand on sait qu'il était de 3,6\% en 1931 et de 3,1\% en 1921. Toutefois, on doit souligner ici que l'évolution des années récentes laisse apparaître un certain déclin de la région dans ce domaine, parallèlement du reste à ce qui se passe dans l'industrie. On rappellera, à cet effet, que la population régionale représentait 5,2\% de celle du Québec, en 1956. Elle a donc enregistré une baisse relative de 0,7\% par rapport à la situation actuelle.

Parallèlement à l'accroissement général de la population, s'est poursuivi un autre mouvement, peut-être plus significatif encore pour le présent propos : l'urbanisation accélérée de la région.

\subsection{La croissance urbaine ou le phénomène de la conurbation du Saguenay}

La croissance en général ne se développe pas de façon uniforme, mais s'opère «par concentration de moyens en des points de croissance dans l'espace » 149. Tout naturellement, le mouvement démographique suit cette évolution. Ainsi naissent et se développent les centres de peuplement, les villes se substituant à l'habitat dispersé traditionnel. Le Saguenay_Lac-St-Jean ne semble pas avoir échappé à cette loi.

Sensiblement le même que pour celui de l'ensemble québécois, le taux d'urbanisation de la région, en 1921, était estimé à 44\%. Mais, déjà dans ce domaine, le comté de Chicoutimi, avec 62\% de sa population considérée comme urbaine, avait pris le pas sur le Lac-St-Jean, dont la population urbanisée était alors de 25\%. La progression du mouvement d'urbanisation (en 1968, $72 \%$ de la population régionale est considérée comme urbaine, contre 79\% pour l'ensemble du 
Québec) appuyé sur l'industrialisation régionale semble avoir consacré de manière irréversible la primauté urbaine de Chicoutimi.

Certes, un phénomène, visible aux yeux de tous les observateurs, caractérise la région : c'est la concentration des principales activités industrielles le long de la rivière Saguenay, entre la Baie des Ha ! Ha !, Port-Alfred, et Kénogami-Jonquière. Cet ensemble, plus ou moins continu de villes, dénommé la «Conurbation du Saguenay », vit en étroite dépendance des entreprises de la Compagnie Alcan, notamment des industries d'aluminium. Ainsi, soulignent certains auteurs, « la dépendance industrielle des villes de la conurbation, c'est-à-dire les emplois dans l'industrie que procure une ville à une autre, n'existe en pratique qu'à l'égard de l'aluminerie d'Arvida » 150. On peut donc dire que le rôle de l'Alcan a été et reste déterminant en ce qui concerne la concentration urbaine et industrielle de la région. Le pourcentage, particulièrement élevé dans certains cas, représenté par les ouvriers d'Alcan dans la main-d’œuvre manufacturière globale des principales agglomérations de la région, ne laisse point de doute à ce sujet. C'est ce qui apparaît au tableau suivant.

Tableau 40

Dépendance industrielle envers Arvida, 1970

$\underline{\text { Retour à la table des matières }}$

\begin{tabular}{|l|c|c|c|}
\hline & La Baie & Chicoutimi & Jonquière / Kénogami \\
\hline Nombre d'ouvriers d'Alcan (n) & 56 & 1839 & 2083 \\
\hline Main-d'œuvre manufacturière totale (N) & 816 & 2487 & 4020 \\
\hline Indice de dépendance : $100 \mathrm{n} / \mathrm{N}$ & 6,8 & 73,9 & 51,8 \\
\hline
\end{tabular}

Source : Louis-Marie Bouchard, op. cit., p. 278.

Les données du tableau précédent laissent voir l'influence exercée par l'Alcan sur les principales villes de la Conurbation, dont Chicoutimi, la capitale administrative régionale, constitue le pôle d'attraction le plus important. D'après une étude du Bureau de Recherches Économiques du

150 L. M. Bouchard, op. cit., p. 278. 
Québec, l'influence de l'agglomération de Chicoutimi «s'étend de l'embouchure du Saguenay jusqu'au Lac St-Jean lui-même, où elle est relayée d'abord par Alma puis par Dolbeau et Roberval » 151. Ainsi, outre l'espace plus ou moins continu représenté par les diverses localités de la conurbation 152, l'Alcan, par l'intermédiaire de son usine d'aluminium d'Alma, étend son influence sur une bonne partie du Lac St-Jean.

Le tableau qui suit a l'avantage de faire ressortir les caractéristiques des centres urbains régionaux et de donner la mesure du poids de la conurbation au sein de l'ensemble économique du Saguenay-Lac-St-Jean.

\section{Tableau 41}

\section{Caractéristiques des centres urbains, région 1966}

$\underline{\text { Retour à la table des matières }}$

\begin{tabular}{|l|c|c|c|c|c|}
\hline & Conurbation & Alma & $\begin{array}{c}\text { Roberval } \\
\text { St-Félicien }\end{array}$ & $\begin{array}{c}\text { Mistassini } \\
\text { Dolbeau }\end{array}$ & Chibougamau \\
\hline \% de la population régionale & 44,4 & 7,9 & 4,8 & 3,8 & 3,0 \\
\hline \% de la main-d'œuvre manufacturière & 67,5 & 12,8 & 2,5 & 4,0 & 6,3 \\
\hline $\begin{array}{l}\text { \% de la main-d'œuvre du commerce } \\
\text { de détail }\end{array}$ & 54,5 & 10,0 & 7,5 & 5,2 & 3,3 \\
\hline \% des ventes du commerce de détail & 51,0 & 14,0 & 8,2 & 6,3 & 3,7 \\
\hline
\end{tabular}

Source : OPDQ, Esquisse de plan de développement, décembre 1969, p. 169.

Tous les éléments qui précèdent montrent l'influence incontestable exercée par l'Alcan sur la formation de la «Conurbation du Saguenay », dont la caractéristique fondamentale est le très haut degré de spécialisation. En effet, avec Alma, où « l'activité secondaire se partage entre l'industrie métallique (Alcan : 54\%) et le bois-papier (38\%), la Conurbation du Haut Saguenay retient plus de 80\% de la main-d’œuvre industrielle régionale » 153. Une

151 BRÉQ, Pôles d’attraction et zones d’influence, MIC, Québec, 1969, p. 19.

152 Voir carte 5.

153 OPDQ, Esquisse de plan de développement, op. cit., p. 166. 
certaine répartition des activités s'est opérée à l'intérieur de la région : «dominante industrielle à l'Ouest (Alma) et à l'Est (la Conurbation), concentration du tertiaire et diverses industries au cœur de l'agglomération (Chicoutimi), plaque tournante des transports » 154. Ainsi prenant le relais de l'industrie papetière et souvent consolidant l'apport de cette dernière, l'Alcan se trouverait à l'origine d'un « pôle industriel complexe qui, géographiquement aggloméré, (a su) modifier son environnement géographique et même l'entière structure de l'économie régionale » 155. Personne n'oserait nier aujourd'hui l'influence déterminante qu'a exercée l'industrie d'aluminium sur la croissance de l'économie du Saguenay_Lac-St-Jean. Mais, l'examen du paysage industriel qu'offre à présent la région, formé de noyaux fortement spécialisés, travaillant en priorité pour le marché extérieur, fait ressortir le besoin urgent qu'a l'économie régionale de se diversifier. Cette remarque amène à considérer les limites du processus de développement de la région à partir de l'Alcan.

154 Idem, p. 169.

155 F. Perroux, L’économie du XXe siècle, op. cit., p. 152. 
Carte 5

Les agglomérations du Saguenay-Lac-St-Jean, 1966

Retour à la table des matières

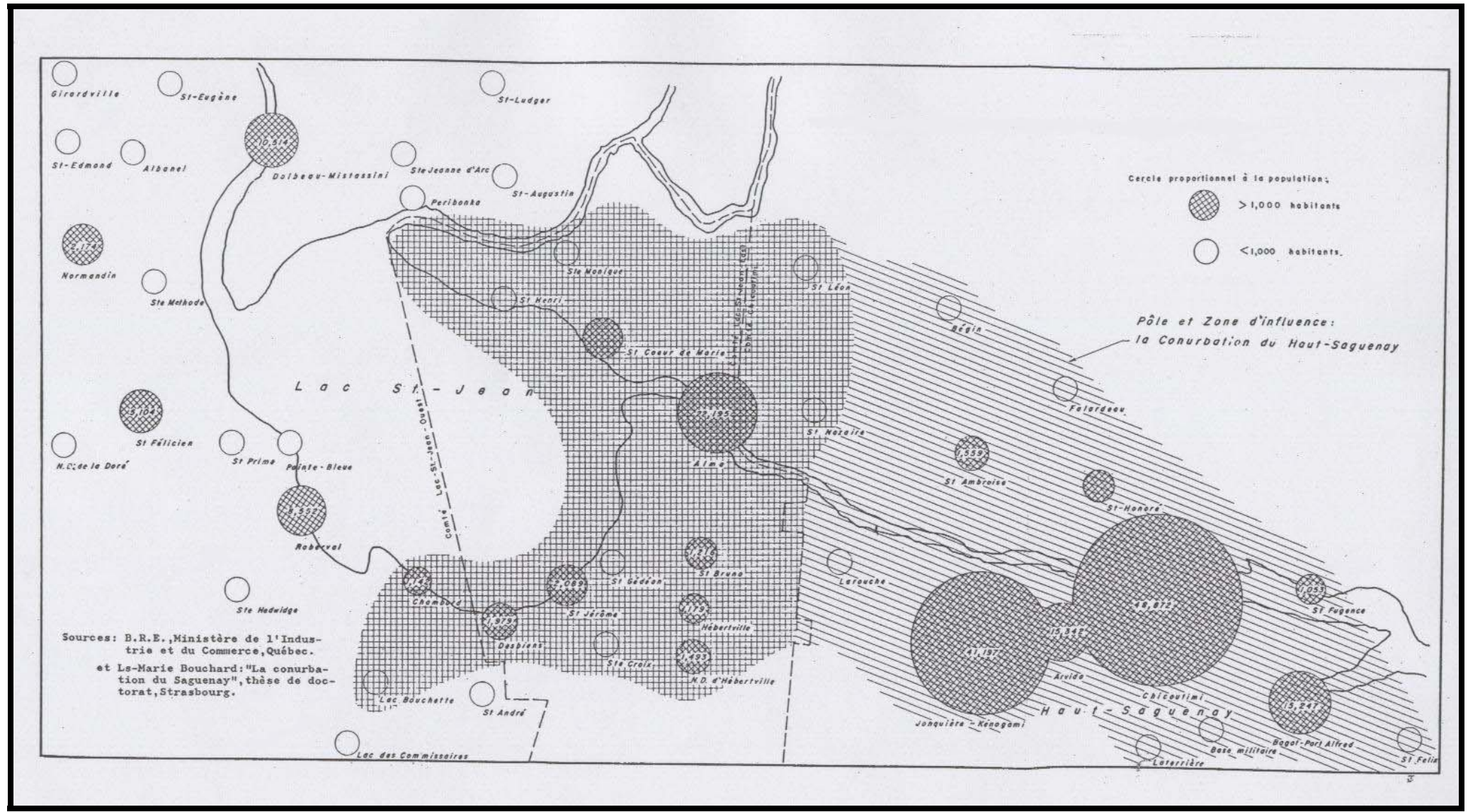


Troisième partie : Le rôle moteur de l’Alcan

Chapitre VII

\section{Les limites du processus de développement du Saguenay- Lac-St-Jean à partir d'Alcan}

$\underline{\text { Retour à la table des matières }}$

Poser le problème des limites d'un processus de croissance ou de développement exige que l'on définisse bien au départ les notions de croissance et de développement. Plus exactement, on devrait plutôt parler de seuil de croissance et de développement, dans la mesure où ces deux phénomènes, essentiellement dynamiques, constituent des mouvements plutôt que des états.

C'est dans cette perspective que l'on doit, semble-t-il, situer la démarche du Professeur Perroux, quand il considère la croissance comme étant « l'augmentation prolongée de la dimension des variables caractéristiques d'une économie, accompagnée de changements de structure et éventuellement de progrès dans l'efficacité de l'effort et dans le bien-être » 156. Il s'agirait donc d'un processus cumulatif, dont le seuil serait le moment où le phénomène devient irréversible parce qu'auto-entretenu. Peu importe la nature des éléments qui se trouvent à

156 F. Perroux, Matériaux pour une analyse de la croissance économique, op. cit., p. 18. 
l'origine du mouvement, l'essentiel étant qu'a un certain moment l'économie puisse se nourrir d'elle-même. C'est ce que d'autres auteurs ont baptisé le « take-off » 157.

On a discuté longtemps pour savoir s'il y a lieu de considérer le « développement » comme un phénomène essentiellement différent de la «croissance ». Sans vouloir entrer dans les subtilités de ces débats, on peut dire que, contrairement à la croissance, le développement déborde largement le cadre de «l'homo oeconomicus » pour envisager l'épanouissement de l'homme dans toutes ses dimensions. Pareille acception, si elle devait être retenue, entraînerait certes les économistes loin du champ familier de leurs raisonnements 158. 11 semble que l'on puisse toutefois se rallier aisément à la notion de Perroux, selon laquelle, « le développement est la combinaisons des changements mentaux et sociaux d'une population qui la rendent apte à faire croître cumulativement et durablement son produit réel global » 159. Quoiqu'il en soit, on peut admettre que le développement, aussi bien que la croissance, exige une productivité croissante, conditionnée par «l'application systématique, généralisée, progressive de la science et de la technologie à la production des biens et des services » 160.

C'est dans les perspectives ainsi définies que, dans ce dernier chapitre, on essaiera de dégager la signification de l'évolution de l'économie du Saguenay_Lac-St-Jean. De manière plus explicite, on tentera de mettre en évidence les limites au processus de développement de cette région et d'analyser les principaux éléments, qui du milieu qui du complexe Alcan, y ont joué un rôle de frein ou d’obstacles.

157 W. W. Rostow, Les étapes de la croissance économique, traduction française, Seuil, Paris, 1962.

158 Ainsi écrit Schumpeter dans la Théorie de l'évolution économique, «Les causes du développement doivent être trouvées dans les seuls faits analysés par la théorie économique ». Cité par Jacques Freyssinet, op. cit., p. 63.

159 F. Perroux, op. cit., p. 155.

160 W. W. Rostow, Industrialization and Economic Growth, Première Conférence Internationale d’Histoire Économique, p. 23. 


\section{Section 1 : Les obstacles provenant du milieu d'accueil}

$\underline{\text { Retour à la table des matières }}$

La première partie de ce travail a été consacré à la présentation du Saguenay—Lac-St-Jean avant l'implantation de l'Alcan. On a alors souligné que la région se situait pratiquement entre l'économie sous-développée et l'économie développée. En tant que telle, l'économie régionale offrait beaucoup de traits propres aux pays sous-développés. Ils se résument, à la vérité, à un problème fondamental de structures que l'on analysera sous les trois rubriques d'économie primaire mal articulée, extravertie et dominée. À ces obstacles fondamentaux s'ajoute, en ce qui concerne le Saguenay_Lac-St-Jean, le problème des ressources humaines.

\subsection{Les structures économiques}

La non-intégration des divers secteurs constitutifs d'une économie, du point de vue de l'homogénéité et de la croissance, s'avère un obstacle majeur. Sous sa forme extrême, cette situation s'identifie au dualisme, où l'on peut voir, « à côté d'une économie industrialisée et évoluée, une économie archaïque, attachée à des modes de vie et d'organisation sociale la maintenant à l'écart de la société nationale»161. Entre les deux secteurs composant l'économie dualiste n'existe pratiquement aucune liaison. Plus exactement, le secteur industrialisé, né généralement sous la poussée de facteurs exogènes, n'entretient avec le secteur traditionnel que « des relations financières par l'intermédiaire des salaires attribués à la main-d'œuvre indigène non-qualifiée et des impôts et redevances versés aux pouvoirs publics » 162. Cette situation ainsi décrite, spécifique aux pays sous-développés, est loin

161 J. Boudeville, Note sur l'intégration des espaces économiques, Cahiers de l’ISEA, série L, no 14, septembre 1964, p. 15.

162 J. Freyssinet, op. cit., p. 260. 
sans doute d'être celle de l'économie du Saguenay-Lac-St-Jean. Mais, elle présentait à coup sûr des défauts structurels analogues, propres à freiner la propagation des effets de croissance.

\subsubsection{L'économie du Saguenay-Lac-St-Jean : une économie primaire mal articulée}

Au moment de l'implantation de l'Alcan dans la région, en 1925, le tableau de l'économie saguenayenne était relativement simple. Comme il a été déjà souligné, l'activité économique reposait principalement sur l'agriculture et l'industrie naissante se réduisait à la transformation du bois en pulpe et en papiers. Jusqu'à un certain point, cette économie de ressources présentait une assez grande homogénéité. Mais, déjà, on doit relever que l'industrie papetière occupait une place privilégiée au sein de l'économie régionale. Or, cette industrie, travaillant très largement pour les marchés extérieurs, n'entretenait que de faibles relations avec les autres secteurs de l'économie de la région. Elle restait tout de même un débouché intéressant pour les producteurs de bois. Débouché faible cependant, dans la mesure où les compagnies productrices de papier avaient leurs propres concessions forestières. Ce qui est certain, c'est que toute cette période a été marquée par la faiblesse de la production régionale, en général, et de la production industrielle, en particulier.

Or, s'il est vrai que «le niveau d'activité de chaque secteur économique dépend non seulement des débouchés finals pour la production du secteur mais conditionne également, dans une plus ou moins grande mesure, les niveaux d'activité des autres secteurs » 163, les automatismes de la croissance, entendus comme la diffusion des effets polarisateurs, ne peuvent s'opérer qu'à l'intérieur d'un réseau dense d'interrelations économiques. Cette condition était loin d'être réalisée, vers 1925, au Saguenay_Lac-St-Jean. Certes, l'implantation dans la région de la puissante machine industrielle de l'Alcan marquera une étape importante dans son évolution. Mais, l'examen de la situation actuelle de l'économie saguenayenne, mieux encore, du secteur industriel, révèle des défauts structurels qui s'apparentent au dualisme.

163 G. Lebel, op. cit., p. 163. 
On peut considérer à cet effet le tableau 42 qui présente la structure du secteur manufacturier régional. Treize groupes majeurs forment cet ensemble, ce qui pourrait constituer le cadre d'une économie relativement diversifiée, sinon équilibrée. Tel n'est pas cependant le cas. En effet, à elle seule, l'industrie métallique primaire, qui se confond largement avec l'aluminium de première fusion, est responsable de près de $60 \%$ de la valeur ajoutée totale de ce secteur et elle fournit plus de 55\% des salaires. Elle occupe environ 48\% de la main-d’œuvre travaillant dans ce secteur et assure environ 60\% de la valeur des expéditions. Au total donc, l'industrie métallique primaire est responsable de la moitié de l'activité industrielle de la région, le reste étant occupé, pour une large part, par l'industrie papetière. On peut donc dire que ces deux branches manufacturières, avec 75\% de l'emploi, 85\% des salaires, 85\% de la valeur des expéditions et 89\% de la valeur ajoutée de l'ensemble du secteur secondaire, constituent bel et bien les piliers de l'activité économique régional et un secteur plus ou moins à part. Incontestablement, ce qui reste de l'activité industrielle de la région, à l'exception de certaines branches, comme les produits alimentaires et boissons, ne pèse pas lourd dans la balance de l'économie du Saguenay_Lac-St-Jean.

Par les moyens financiers dont elles disposent, la technologie mise en oeuvre et la productivité qui en découle, le niveau moyen des salaires offerts, les industries d'aluminium et du papier font contraste avec le reste de l'économie saguenayenne. À ce propos, il est intéressant d'examiner le tableau 43 qui fait la comparaison des moyennes de salaires annuels et de valeur ajoutée par employé des différents groupes du secteur manufacturier régional. Si le terme « dualisme » ne s'applique pas ici, il est cependant possible de parler certainement de disparité. Il est vrai que le Saguenay_Lac-St-Jean n'est pas la seule région à être dans le cas, puisque, de l'avis de G. Lebel, « l'observation des vingt dernières années laisse une impression dominante : la croissance de l'économie du Québec a été très variable d'un secteur à l'autre et a été accompagnée de disparités fondamentales » 164.

164 G. Lebel, op. cit., p. 136. 
Tableau 42

Secteur manufacturier :

poids relatif des groupes majeurs, région 1965

(en pourcentage)

$\underline{\text { Retour à la table des matières }}$

\begin{tabular}{|l|c|c|c|c|}
\hline Groupes majeurs & Emploi & Salaires & $\begin{array}{c}\text { Valeur des } \\
\text { expéditions }\end{array}$ & $\begin{array}{c}\text { Valeur } \\
\text { ajoutée }\end{array}$ \\
\hline 1. Aliments et boissons & 10,1 & 6,1 & 7,5 & 4,2 \\
\hline 2. Cuir & 0,3 & 0,2 & 0,1 & 0,2 \\
\hline 3. Vêtement & 0,2 & 0,1 & 0,03 & 0,03 \\
\hline 4. Bois & 9,5 & 5,1 & 4,8 & 5,14 \\
\hline 5. Meubles & 0,8 & 0,5 & 0,3 & 0,3 \\
\hline 6. Papiers et produits connexes & 26,3 & 29,3 & 26,4 & 28,6 \\
\hline 7. Imprimerie, édition et connexes & 1,3 & 0,9 & 0,4 & 0,6 \\
\hline 8. Industrie métallique primaire & 48,2 & 55,3 & 58,9 & 59,9 \\
\hline 9. Industrie des produits métalliques & 1,68 & 1,25 & 0,61 & 0,5 \\
\hline 10. matériel de transport & 0,1 & 0,03 & 0,01 & 0,01 \\
\hline 11. produits minéraux non-métalliques & 1,3 & 1,1 & 0,9 & 0,7 \\
\hline 12. Industrie chimique & 0,02 & 0,02 & 0,01 & 0,01 \\
\hline 13. manufactures diverses & 0,2 & 0,1 & 0,04 & 0,01 \\
\hline
\end{tabular}

Source : Mission de planification du Saguenay_Lac-St-Jean : Situation actuelle et évolution récente de l'industrie manufacturière, MIC, Québec, août 1969, p.28. 


\section{Tableau 43 \\ Salaire annuel moyen et valeur ajoutée par employé dans les industries manufacturière Saguenay_Lac-St-Jean 1965}

\section{$\underline{\text { Retour à la table des matières }}$}

\begin{tabular}{|l|c|c|}
\hline Groupes majeurs & $\begin{array}{c}\text { Salaire } \\
\text { (milliers de \$) }\end{array}$ & $\begin{array}{c}\text { Valeur ajoutée } \\
\text { (milliers de \$) }\end{array}$ \\
\hline Aliments et boissons & 3,5 & 6,9 \\
\hline Cuir & 3,3 & 8,5 \\
\hline Vêtement & 2,4 & 4,1 \\
\hline Bois & 3,1 & 9,2 \\
\hline Meubles & 3,5 & 6,3 \\
\hline Papiers et produits connexes & 6,5 & 18,6 \\
\hline Imprimerie, édition et produits connexes & 3,9 & 7,4 \\
\hline Industrie métallique primaire & 6,7 & 21,2 \\
\hline Produits métalliques & 4,4 & 4,4 \\
\hline Matériel de transport & 2,1 & 2,5 \\
\hline Produits minéraux non-métalliques & 4,8 & 9,7 \\
\hline Industrie chimique & 5,3 & 6,1 \\
\hline Manufactures diverses & 2,3 & 5,0 \\
\hline Total des groupes & 5,8 & 17,1 \\
\hline Total des groupes moins aluminium et papier & 3,5 & 7,7 \\
\hline
\end{tabular}

Source : Mission de planification du SLSTJ, op. cit., p. 59.

L'asymétrie évidente de la structure industrielle régionale révélée par les données du tableau précédent se confirme, si on se réfère au poids des établissements manufacturiers. Il a été souligné ailleurs que seulement cinq établissements, appartenant aux industries d'aluminium et du papier, soit $1,7 \%$ de l'ensemble du secteur, fonctionnent avec 500 employés ou plus. Si ceux-là s'inscrivent dans la dynamique des grandes entreprises contemporaines, tel n'est pas le cas des établissements n'employant que quatre personnes ou moins, que l'on peut à juste titre considérer comme opérant sur des bases artisanales. Or, « en 1965, 50\% des établissements manufacturiers de la région se retrouvaient dans ce groupe, ne contribuant que pour $2 \%$ à 
l'emploi, $0,8 \%$ aux salaires, $1,1 \%$ à la valeur des expéditions et $0,7 \%$ à la valeur ajoutée de l'ensemble du secteur industriel » 165.

Ainsi, jusqu'à un certain point, le développement des deux secteurs-clés de l'industrie régionale a joué le rôle de facteurs déséquilibrants. Mais, comme dit Le Senne, «tout obstacle est un appel »166. Et, s'il est vrai que la croissance est « une séquence de déséquilibres » 167 ou un processus de « challenge and response » 168, l'économie régionale se trouverait en présence d'un défi hautement stimulant et donc favorable à son développement. Encore faudrait-il que les wagons du train économique soient bien attachés à ses locomotives. L'analyse qui suit essaiera de faire la lumière sur ce problème fondamental.

\subsubsection{L'économie saguenayenne :} une économie extravertie et dominée

On a souligné précédemment que l'économie du Saguenay—Lac-St-Jean se caractérise par un très haut degré de spécialisation doublé d'une grande faiblesse au niveau des liaisons entre les divers secteurs et branches de l'activité économique et industrielle. Cette absence de diversification dans la production régionale entraine une conséquence majeure : la polarisation en quelque sorte de l'économie régionale par l'extérieur, que ce soit par le pôle de développement que constitue l'agglomération montréalaise, que ce soit par les grands marchés mondiaux. De ce point de vue, le «Royaume du Saguenay » est loin d'être, comme on pourrait le croire de prime abord, une région repliée sur elle-même et vivant en circuit fermé.

Du reste, cette réalité n'est pas à déplorer, car l'économie ouverte, basée sur les échanges, représente certainement un progrès par rapport à l'autarcie. Mais, on ne peut tout de même pas ignorer les dangers que court une économie qui tire l'essentiel de ses ressources d'une gamme étroite de produits et qui, de surcroît, vit en dépendance directe de l'extérieur pour ses approvisionnements ou l'écoulement de sa production. L'illustration frappante de cette idée est le

165 Mission de planification économique du SLSJ, op. cit., p. 6.

166 Cité dans F. Perroux, L'économie du XXe siècle, p. 263.

167 A. O. Hirschman, Stratégie du développement économique.

168 P. Streeten, Economic Integration, Sythoff, Leyden, 1961. 
cas de Val-Jabert bien connu des gens de la région. Village jadis prospère du Lac St-Jean, ValJabert s'est transformé en village-fantôme le jour même, 13 août 1927, où la Compagnie Price décida la fermeture de son moulin à papier, la principale sinon la seule activité de la localité.

\subsubsection{Le Saguenay_Lac-St-Jean et la région montréalaise}

Au point de vue spatial, tous les observateurs s'accordent à reconnaître qu'un trait marquant de l'économie du Québec est la profonde disparité entre les diverses régions. En cela, du reste, l'économie québécoise ne fait que refléter une situation généralement observable au niveau de l'économie du Canada tout entier. Il est un fait admis que «l'économie canadienne repose sur des contreforts gothiques, constitués par des échanges et financements extérieurs, représentant plus du quart du corps de l'édifice. En outre, ses relations économiques extérieures dépendent en majeure partie d'un seul pays : les États-Unis » 169.

En ce qui concerne la Province du Québec, il n'est pas de régions qui, directement ou indirectement, ne ressentent le poids de la domination de la puissante agglomération métropolitaine. Montréal exerce une influence prépondérante sur l'ensemble de l'économie provinciale. En dépit de sa relative excentricité. géographique, le Saguenay_Lac-St-Jean ne semble pas échapper à cette réalité. Une première mesure de la primauté de la région montréalaise au sein de l'économie québécoise peut être prise à travers les données concernant l'apport relatif des régions à l'activité industrielle 170.

À tous les titres, l'industrie de la région de Montréal représente plus de deux tiers de celle de l'ensemble des dix régions administratives du Québec. Compte tenu de l'immensité du territoire de cette province, cette donnée indique qu'en dehors de Montréal, l’activité industrielle est faible et dispersée.

169 J. Boudeville, Note sur l’intégration des espaces économiques, op. cit., p. 28.

170 Voir le tableau 44. 
Tableau 44

\section{Les industries manufacturières du Québec par régions administratives, 1964}

$\underline{\text { Retour à la table des matières }}$

\begin{tabular}{|l|c|c|c|c|}
\hline Régions & $\begin{array}{c}\text { Main-d’ouvre } \\
\text { employée }\end{array}$ & $\begin{array}{c}\text { Gages et salaires } \\
\text { (milliers de \$) }\end{array}$ & $\begin{array}{c}\text { Expéditions } \\
\text { (milliers de \$) }\end{array}$ & $\begin{array}{c}\text { Valeur ajoutée } \\
\text { (milliers de \$) }\end{array}$ \\
\hline Bas-St-Laurent Gaspésie & 5654 & 15897 & 98151 & 33622 \\
\hline Saguenay-Lac-St-Jean & 14542 & 81326 & 432654 & 229042 \\
\hline Québec & 42885 & 158430 & 692904 & 347739 \\
\hline Trois-Rivières & 42307 & 168030 & 799365 & 396110 \\
\hline Cantons de l’Est & 21480 & 82502 & 385722 & 179369 \\
\hline Montréal & 319886 & 1363897 & 6260268 & 2834160 \\
\hline Outaouais & 9504 & 42908 & 206118 & 103625 \\
\hline Nord-ouest & 4356 & 16222 & 204662 & 41303 \\
\hline Côte-Nord, Nord-du-Québec & 2693 & 15827 & 95438 & 48060 \\
\hline Total province & 463307 & 1945040 & 9175181 & 4213931 \\
\hline
\end{tabular}

Source : Saguenay-Lac-St-Jean, industries manufacturières, Publication no 12, décembre 1965.

Il n'est certes pas exclu de rencontrer deci-delà des îlots d'industrialisation. Mais, de véritable pôle industriel de développement, créateur de complémentarités multiples et variées, il n'en existe qu'un : celui de la Métropole de Montréal et de ses environs. Encore que des auteurs, comme Martin et Raynauld, estiment que Montréal, en tant que pôle, est faible et menacé 171.

Quoiqu'il en soit de la situation relative de Montréal face aux pôles industriels de l'Amérique du Nord, il ne fait aucun doute que les régions du Québec, en général, et le Saguenay, en particulier, ne font pas le poids à côté de la métropole, dont elles subissent la domination.

171 F. Martin et A. Raynauld, « Les choix urbains et régionaux dans le Québec des années 1970 », dans Le Québec d'aujourd'hui, op. cit., p. 203. [Texte disponible dans Les Classiques des sciences sociales. JMT.] 
Cette domination s'exerce à travers des mécanismes bien connus et qui, à quelques différences près, se définissent par le type de relations qu'entretiennent toutes les métropoles avec leur hinterland. Par définition, la grande métropole, par la multiplicité de ses centres d'attraction et de loisirs, par la diversité de ses activités tant industrielles que tertiaires, joue comme une pompe aspirante, drainant les éléments les plus jeunes et les plus dynamiques de sa zone d'influence. Le niveau moyen de salaire de la métropole étant plus élevé que celui des autres régions, les économies externes y étant abondantes ou, en tout cas, plus avantageuses, l'arrière-pays se trouve ainsi défavorisé. Toute une série de facteurs économiques et psychologiques travaillent dans le sens d'une surexploitation de la région métropolitaine au détriment des régions périphériques ou excentriques.

En ce qui concerne le Saguenay_Lac-St-Jean, on a souligné ailleurs que la tendance des dernières années a été dans le sens d'une émigration d'une partie de la population vers l'extérieur. Ainsi, entre 1961 et 1966, plus de 21500 personnes ont abandonné la région pour s'installer ailleurs, soit 8,2\% de la population 172. I1 est par ailleurs significatif que la plupart des entreprises importantes de la région, comme l'Alcan, aient leur siège social à Montréal. L'économie régionale est ainsi plus ou moins dirigée de l'extérieur, sans compter que la région dépend de Montréal pour une bonne partie de ses approvisionnements.

Ces considérations n'entraînent pas forcément que, dans une optique de politique de développement régional au Québec, l'agglomération montréalaise devrait être négligée au profit des autres régions, plus ou moins déprimées, comme le Saguenay-Lac-St-Jean. À court terme, la consolidation de la position concurrentielle de Montréal en Amérique du Nord implique certes « le renforcement du pôle de développement qu'est la région de Montréal » 173. Mais, on ne peut s'empêcher de penser qu'une telle politique, si elle doit se faire au détriment des autres régions, entraînerait des conséquences néfastes à long terme, dont l'aggravation des

172 Journal Le Travail, organe officiel de la Confédération des Syndicats nationaux (CSN), Montréal, juillet 1971.

173 Higgins, Martin, Raynauld, Les orientations du développement économique régional dans la province de Québec, Ministère de l’Expansion Économique Régionale, 1970. 
disparités observables ne serait pas la moindre. Cela serait d'autant plus grave que ces régions, comme on l'a souligné, restent confinées dans le rôle essentiel de centres d'exploitation d'un ou de deux produits de base, dont le marché déborde de toute manière, largement, le cadre de Montréal. Leurs économies échapperaient ainsi à la causalité interne, même élargie.

\subsubsection{Le Saguenay—Lac-St-Jean et l'extérieur}

I1 a été maintes fois souligné, dans la présente étude, que l'économie du Saguenay—LacSt-Jean repose essentiellement sur ses deux importantes industries d'aluminium et du papier. On sait que la majeure partie de ces deux productions régionales trouve leurs débouchés en dehors du Canada. Ainsi, en 1961, 62,8\% de la production globale de ces deux secteurs devaient être écoulés sur les marchés internationaux, contre seulement 28,5\% sur les marchés de la province du Québec et 8,7\% dans les autres parties du Canada. Compte tenu du fait déjà souligné que la structure économique régionale a peu changé, ces données fournissent des ordres de grandeur largement valables encore aujourd'hui. Les mêmes observations peuvent être faites à propos de l'industrie des produits alimentaires, l'activité agricole étant axée sur la production des produits laitiers, en particulier du fromage.

Or, on sait que «plus un pays est ouvert au monde extérieur, plus l'élasticité de ses importations est forte, ce qui entraîne d'importantes fuites de revenus et, par conséquent, un faible multiplicateur. Ce pays reçoit bien et retransmet quasi intégralement les fluctuations d'origine extérieure » 174. Ces considérations de P. Moran s'appliquent parfaitement à la région, à la différence que, contrairement à un pays, le Saguenay-Lac-St-Jean ne dispose pas des instruments que la souveraineté économique lui donnerait pour faire efficacement face à ces variations de la conjoncture.

L'économie du Saguenay_Lac-St-Jean, spécialisée à outrance et tributaire des échanges extérieurs, s'avère ainsi éminemment vulnérable. Dominée par les marchés internationaux, elle répercute les moindres soubresauts de la conjoncture internationale, comme on l'a déjà montré pour la crise des années 1930 et la seconde guerre mondiale. À la vérité, le caractère

174 P. Moran, op. cit., p. 177. 
extraverti de l'économie saguenayenne est inhérent à tous les « espaces non-concordants » où, selon J. Paelinck, «la densité de l'espace géographique relative à l'espace économique est proportionnellement plus élevée, c'est-à-dire où le réseau des relations économiques dans l'espace géographique est peu dense et peu développé » 175. On a montré plus haut le rachitisme des rapports entretenus par les deux principales branches industrielles du Saguenay-Lac-St-Jean avec le reste de l'économie. Il convient maintenant de se pencher sur le second aspect de la non-concordance de l'espace saguenayen : la densité démographique ou, de manière plus large, le problème humain.

\subsection{Le problème humain}

Retour à la table des matières

S'il est vrai que «la dimension d'une nation (ou d'une région) constitue une véritable caractéristique structurelle de son économie »176, c'est non seulement par référence aux ressources naturelles qu'elle offre, mais surtout aux populations qui y vivent. Sous le double aspect quantitatif et qualitatif, l'élément humain constitue une caractéristique majeure de l'économie d'une région. Il est vrai que toute richesse demeure stérile, laissée à elle-même, de la même façon que tout bien n'a en définitive de valeur qu'en référence aux hommes. De là à vouloir établir des échelles de combinaisons plus ou moins optimales entre les hommes et les ressources il n'y a qu'un pas qui ne sera pas franchi dans le cadre de la présente étude. Cependant, on tentera de montrer comment le facteur humain, agent fondamental de la production et composante essentielle du marché, a influencé dans un sens négatif la réponse du milieu à l'impulsion de l'Alcan.

\subsubsection{La faiblesse de la main-d'œuvre}

On a analysé ailleurs 177 cette importante caractéristique des structures socio-économiques du Saguenay_Lac-St-Jean. Il a été alors montré que l'insuffisance numérique de la main-

175 J. Paelinck, Croissance régionale et mise en valeur des ressources en Amérique Latine, dans Cahiers de l'ISEA,L-13, octobre 1963, p. 21.

176 P. Moran, op. cit., p. 212.

177 Voir plus haut La démographie régionale. 
d’œuvre procède du phénomène général de la faiblesse de la densité démographique. Il ne fait aucun doute que la faiblesse de la main-d’œuvre peut être un élément de freinage sinon de blocage de la croissance économique. Mais, en ce qui concerne le Saguenay-Lac-St-Jean, cette proposition, à première vue, parait pour le moins paradoxale, compte tenu du taux particulièrement élevé de chômage qui sévit dans la région 178. À la vérité, quand on considère une période relativement longue, soit celle qui s'étend de 1925 à nos jours, on se rend compte que la demande régionale de main-d’œuvre a été en moyenne beaucoup plus forte que l'offre. On en a la preuve dans les afflux importants de main-d'œuvre extérieure qui ont caractérisé toutes les phases principales de l'industrialisation de la région, comme en 1925-26 et, plus tard, en 1943. Cela explique que la population régionale s'est accrue à un rythme relativement plus rapide que celui de l'ensemble de la province du Québec. Mais, précisément, une partie nonnégligeable de la population et de la main-d'œuvre se révèlera plus sensible aux variations de la conjoncture économique régionale. Il en découle un important facteur d'instabilité au niveau de la masse de main-d’œuvre disponible.

Il faut en outre souligner que l'absence de véritables traditions industrielles dans la région n'invitait pas cette main-d'œuvre à une qualification technique poussée. De la même façon, les « élites » de la région n'étaient ni formées ni prédisposées à se lancer dans l'aventure, passionnante pour d'autres, de l'industrialisation. Cette faiblesse de «l'entrepreneurship », qui n'est pas d'ailleurs spécifique à la région 179, ajoutée au manque de qualification de la maind'œuvre, a été un obstacle certain à la diffusion des effets de croissance des pôles potentiels. Mais, s'il est vrai que « exception faite de certains secteurs marginaux, l'instruction a toujours été une préoccupation majeure des habitants du Saguenay-Lac-St-Jean »180, on peut penser que ces obstacles seront résorbés dans le moyen terme, à la faveur du mouvement de refonte et de réorientation du système d'enseignement qui anime le Québec depuis 1960.

178 En 1970 selon l'organe officiel de la Confédération des Syndicats nationaux, le taux moyen de chômage dans le Saguenay-Lac-St-Jean a été de 14 \%.

179 Voir à ce sujet N. W. Taylor, A Study of French Canadians as Industrial Entrepreneurs, Thèse de doctorat, Université Yale, 1957.

180 P.-Y. Pépin, op. cit., p. 139. 
Aujourd'hui, avec son réseau de CEGEP, d'écoles techniques et le nouveau campus universitaire implanté dans la capitale régionale 181, il ne fait pas de doute que le Saguenay_Lac-St-Jean est mieux armé pour faire face au problème de la qualification de sa main-d’œuvre. Du reste, certains observateurs relèvent que dans la région « la fréquentation scolaire au niveau secondaire et post-secondaire est proportionnellement plus forte que pour l'ensemble du Québec » 182. C'est là un indice encourageant pour l'avenir. Il n'en reste pas moins que les lacunes au niveau de la qualification de la main-d'œuvre ont joué historiquement un rôle de frein pour la croissance économique régionale. Certes, le problème majeur de la région est actuellement celui d'une économie incapable d'absorber la main-d’œuvre disponible, ce qui s'explique en grande partie par le fait, déjà souligné, du peu de diversification des activités industrielles régionales. Cette caractéristique de l'économie saguenayenne est à mettre en relation avec la faiblesse du marché régional, qu'il convient maintenant d'analyser de façon plus systématique.

\subsubsection{Le marché de la région du Saguenay-Lac-St-Jean}

Il est presque banal de dire que, dans le cadre du système économique libéral, le marché est à l'activité économique ce que la nécessité est à l'invention. Génératrice possible de profits, la demande solvable, elle-même liée à la demande potentielle, constitue un véritable aiguillon, poussant à entreprendre et à innover. De manière négative, l'étroitesse du marché peut jouer le rôle de facteur prohibitif à l'égard de certains types d'industries. En ce sens, le marché peut être considéré comme l'un des principaux moteurs de l'expansion économique.

Traitant des structures de l'économie du Saguenay_Lac-St-Jean, on a mis en relief le double phénomène du quasi monolithisme de l'appareil de production industrielle et de la dépendance à l'égard des grands marchés internationaux. Cette double caractéristique de l'économie régionale s'explique en partie par la faiblesse du marché saguenayen.

181 CEGEP : Collège d’Enseignement Général et Professionnel. L’Université du Québec à Chicoutimi a commencé ses activités avec l’année académique 1969-1970.

182 P.-Y.Pépin, op. cit., p. 139. 
Il est certes évident que, même estimée à 300000 habitants, la population actuelle de la région ne forme pas un marché potentiel apte à soutenir ou à engendrer une industrie diversifiée et puissante. Cela est d'autant plus vrai que, comme on l'a souligné, cette population est relativement jeune. Cela entraîne une charge particulièrement lourde pour la population active, ou plus exactement, pour la main-d'œuvre effectivement au travail. Ainsi, le taux de dépendance mesuré par le rapport,

\section{population de 0 à 14 ans + population de 65 ans et + population totale}

était de 40,2\%, en 1964 183. Ce premier aspect de l'étroitesse du marché régional saguenayen a certainement joué un rôle de freinage dans l'implantation des entreprises d'aval de l'aluminium. Certes, il est évident que l'influence de ce facteur est médiatisée par les entreprises industrielles existantes ou potentielles. En clair, cela signifie que la première composante du marché du produit primaire que représente l'aluminium est la demande des entreprises régionales de transformation. Mais, l'implantation ou le développement de ces dernières est elle-même conditionnée par la demande finale. Cela amène à considérer le deuxième volet de la faiblesse du marché régional saguenayen.

Il s'agit, on s'en doute, du volume de pouvoir d'achat qui échoit à l'ensemble de la population régionale. En 1966, le revenu total disponible des particuliers s'élevait à près de 390 millions de dollars, soit un revenu per capita de 1380 dollars. Dans ce domaine, le SaguenayLac-St-Jean semble relativement défavorisé, puisque, pour la même année, le revenu disponible par tête, pour l'ensemble de la province du Québec, était de 1705 dollars. De fait, le Saguenay n'occupait que la huitième place parmi les dix régions du Québec. Il conviendrait encore de déterminer la partie de la masse de revenus disponibles, au sein de la région, qui va vers l'achat de produits finis à base d'aluminium. En l'absence de données statistiques, cette tâche s'avère impossible. Quoiqu'il en soit, on peut penser que ces dépenses participent à la faiblesse du marché potentiel et du pouvoir d'achat disponible dans la région.

183 Selon l’Office de Planification et de Développement du Québec, op. cit., p. 10. 
Ainsi, en termes de demande potentielle aussi bien que de demande solvable, le marché régional saguenayen n'offre pas de réels débouchés à l'industrie d'aluminium primaire. Or, la relative excentricité géographique de la région par rapport aux grands centres industriels et aux marchés importants de consommation, tels que Montréal et Toronto, n'est pas contrebalancée, même par la proximité de la source d'approvisionnement en matières premières ou l'abondance de l'énergie hydroélectrique. Il est du reste significatif que la Compagnie Alcan n'ait pas pensé développer dans la région le secteur de transformation de l'aluminium en produits finis. On peut également souligner que d'autres entreprises qui auraient été intéressées à s'implanter dans le Saguenay-Lac-St-Jean ont peut-être été effrayées par la puissance de l'Alcan. Cela conduit à examiner la seconde catégorie d'obstacles à la pleine diffusion des effets de polarisation du complexe électro-métallurgique, inhérents à la nature même de l'Alcan.

\section{Section 2 : Les obstacles provenant de l'Alcan}

\section{$\underline{\text { Retour à la table des matières }}$}

Dans la deuxième partie de la présente étude 184, on a montré que l'Alcan, par la grande dimension de ses activités et la multiplicité des territoires dans lesquels elle opère, est avant tout une grande unité inter-territoriale. À la vérité, si, comme on l'a déjà souligné, la Compagnie n'est pas « une monocoque isolée au sein de la région », il n'en reste pas moins que le Saguenay-Lac-St-Jean appartient à l'Alcan beaucoup plus que l'Alcan n'appartient au Saguenay_Lac-St-Jean. En effet, la présence de cette société au sein de l'économie de la région ne s'explique que par les abondantes ressources hydroélectriques du Saguenay. Même grotesque dans la réalité, l'hypothèse, que l'Alcan pourrait reconvertir ailleurs ses activités régionales, s'avère largement concevable. Jusqu'à un certain point donc, et sans connotation péjorative, on peut dire que la région du Saguenay_Lac-St-Jean a besoin beaucoup plus de l'Alcan que cette dernière n'a besoin d'elle. Dans ce contexte, on est en droit de se demander si 
la stratégie propre de la Compagnie n'a pas pu constituer un frein à la diffusion de ses effets particuliers de croissance.

\subsection{La théorie du comportement de la GUI}

\section{$\underline{\text { Retour à la table des matières }}$}

C'est au Professeur M. Byé que l'on doit l'expression de « grande unité inter-territoriale » par laquelle il désigne « un ensemble organisé de moyens de production soumis à un centre de décision unique, capable d'autonomie à l'égard du marché et contrôlant divers établissements productifs, situés sur plusieurs territoires nationaux »185. De par sa dimension, l'entreprise multinationale établira généralement sa stratégie indépendamment des problèmes spécifiques de tout milieu particulier d'implantation. Du reste, la plupart des établissements de la GUI constituent « des intégrations industrielles originaires des pays (ou régions) développés qui ont cherché en des territoires sous-développés divers leurs sources de matières premières ou d'énergie » 186.

Ces éléments, à eux seuls, contribuent à donner à la grande unité inter-territoriale un comportement spécifique, selon qu'elle se réfère à une région d'implantation ou à son milieu d'origine.

\subsubsection{La GUI et son milieu d'origine}

Dans la mesure où ses capitaux proviennent en quasi totalité de son milieu d'origine, la grande unité inter-territoriale y installera son siège social. C'est là que seront prises toutes les décisions majeures concernant l'évolution de la société, le volume et la nature des investissements qui assureront son expansion, le degré d'exploitation de ses immobilisations ou de ses sources de matières premières, sa politique en matière de personnel et d'embauche, le niveau des prix de ses produits. Non seulement, la politique généralement appliquée dans

185 M. Byé, Relations économiques internationales, 2 édition, Dalloz, 1965, p. 233.

186 M. Byé, Encyclopédie française, tome IX, L’Univers économique et social, p. 9-10-4. 
chaque établissement est élaborée dans le milieu de provenance de la GUI, mais encore, elle est basée sur des données globales et en fonction d'objectifs précis qui débordent, et souvent largement, le cadre des réalités propres de chaque région particulière. Comme le souligne le Professeur Byé, «le centre de gravité de la GUI étant situé en dehors des régions, son comportement sera entièrement subordonné à ses besoins tels qu'ils y sont perçus » 187.

Compte tenu du lieu de provenance des capitaux, les profits réalisés par la grande unité inter-territoriale enrichissent le milieu d'origine plus que les régions d'implantation. Du reste, les profits sont difficilement identifiables, comme provenant de tel établissement. C'est que « le revenu de la grande unité inter-territoriale doit être considéré comme un tout, quel que soit son lieu de provenance. Il dépend donc du plan général de la GUI que les recettes en provenance de tel territoire soient transférées pour être investies sur tel autre territoire » 188. On peut d'ailleurs ajouter que généralement les investissements effectués dans tel établissement particulier de la GUI profitent, souvent, moins au territoire d'implantation qu'au milieu d'origine qui fournit pratiquement tout l'outillage ou les équipements techniques nécessaires. Quel est alors le contenu des rapports entre une grande unité inter-territoriale et ses territoires d'implantation?

\subsubsection{La GUI et les territoires d'implantation}

L'analyse qui précède légitime une hypothèse, du reste, généralement vérifiée dans la réalité, à savoir que l'établissement d'une grande unité inter-territoriale constitue le plus souvent une enclave, un flot, au sein de l'économie du territoire d'implantation. En effet, « secteur de financement clos », la firme membre d'une GUI s'intègre mieux à l'économie dominante du milieu d'origine. C'est que l'essentiel des rapports entre la grande unité inter-territoriale et une région d'implantation s'exerce souvent à travers l'embauche de main-d'œuvre et l'utilisation des matières premières ou de l'énergie que le milieu d'accueil lui fournit, moyennant les salaires et les revenus fiscaux distribués. Encore convient-il de souligner que la majeure partie du

187 M. Byé, Cours de doctorat, Faculté de Droit de Paris, 1954, p. 235.

188 M. Byé, «L'autofinancement de la GUI et les dimensions temporelles de son plan », dans Revue d'Économie Politique, mai-juin 1957, p. 302. 
personnel administratif et des cadres techniques supérieurs de l'établissement de la GUI provient de milieu d'origine.

Par la dimension de ses activités et des moyens mis en oeuvre, faisant souvent contraste avec les entreprises traditionnelles du territoire d'implantation, la grande unité inter-territoriale entraîne un accroissement de la production et des modifications parfois importantes des structures économiques de ce dernier. Mais, la densité et l'orientation des activités de l'entreprise de la GUI échappent complètement à l'influence du milieu d'implantation. À court terme, elles dépendent surtout de la situation du marché du produit qui, le plus souvent, est extérieur à la région d'accueil et relève du pouvoir des économies « étrangères » dominantes. À long terme, le territoire d'implantation n'exerce pas plus un pouvoir de décision quant aux activités de ce type d'entreprise qui « dépend alors de la durée de plan adoptée par la GUI qui peut même être en conflit avec celle du territoire d'établissement » 189.

Il est certes incontestable que le territoire d'implantation retire des avantages significatifs de la présence de la GUI, ne fût-ce parce que cette dernière permet l'exploitation de richesses qui seraient restées autrement stériles. Les revenus créés et distribués peuvent être à l'origine d'un processus multiplicateur au sein de l'économie du territoire d'implantation. Mais, le commerce extérieur de la grande unité inter-territoriale peut contribuer au « vieillissement indu de la structure externe du pays d'implantation, par une balance excédentaire, le placement de capitaux et le paiement de revenus au-dehors, comme une économie développée » 190. En définitive, on peut se demander si la grande unité inter-territoriale, riche contribuable aussi bien qu'espace de plan autonome, peut être un levier de transmission de croissance. À cette question, la théorie ne saurait fournir une réponse absolue et universelle. On ne peut, à ce niveau, que souligner les dangers de déséquilibre ou de dualisme inhérents à l'implantation d'une GUI dans une économie à structures simples et lâches. Seule l'analyse des cas concrets permettra d'apporter des réponses particulières à la question posée. On est ainsi conduit à considérer la situation de la GUI qu'est l'Alcan par rapport à l'économie saguenayenne.

189 M. Byé, L'autofinancement de la GUI, op. cit., p. 299-300.

190 P. Aydalot, op. cit., Cahiers de l'ISEA, p. 140. 


\subsection{L'Alcan - GUI et l'économie saguenayenne}

$\underline{\text { Retour à la table des matières }}$

On a déjà largement analysé les effets positifs de la Société sur l'évolution de l'économie du Saguenay_Lac-St-Jean. Le moment est venu d'examiner si et en quoi la présence de l'Alcan a pu porter préjudice à l'évolution du « Royaume du Saguenay ». Il convient peut-être de préciser au départ que cette démarche ne vise en aucune manière à poser de quelconques jugements de valeur quant aux comportements ou aux stratégies de cette Compagnie. De façon spécifique, on doit admettre avec le professeur Perroux « qu'à l'échelle internationale comme partout ailleurs, l'effet de domination n'a aucun rapport direct avec un jugement de valeur ou une prise de position doctrinale » 191. Cela dit, on analysera dans un premier temps les effets de domination et de monopole exercés par l'Alcan et dans un second temps les déséquilibres structurels et conjoncturels qui en résultent pour l'économie saguenayenne.

\subsubsection{Les effets de domination et de monopole de l'Alcan sur l'économie du Saguenay-Lac-St-Jean}

Depuis que Chamberlin a ouvert la voie par son « entreprise révolutionnaire » 192, toute une littérature s'est développée sur «les structures non-concurrentielles et asymétriques » 193 ou les économies dominantes. À la vérité, le problème n'est pas bien nouveau, même si les économistes, perdus dans la contemplation de leur modèle idéal de concurrence pure et parfaite, n'avaient pas vu que l'économie, comme partout ailleurs dans la nature et la société, offrait un domaine privilégié où s'exercent des forces différentes, avec des motivations souvent divergentes. Qu'est-ce en effet que l'effet de monopole ou de domination ? « Intentionnel ou non », répond le professeur Perroux, ce n'est rien d'autre que «l'influence dissymétrique ou irréversible exercée par une unité sur une autre, en raison de sa

191 F. Perroux, L'économie du XXe siècle, op. cit., p.93.

192 E. H. Chamberlin, The Theory of Monopolistic Competition, Harvard, 1933, PUF, Paris, 1953.

193 M. Byé, Les relations économiques internationales, op. cit., chapitre V, pp. 222-249. 
dimension, de son pouvoir de négociation, de la nature de son activité ou de son appartenance à une zone d'activité dominante » 194.

En ce qui concerne l'Alcan, tous les développements qui précèdent montrent qu'elle présente une profonde dissymétrie par rapport aux autres unités ou secteurs de production de l'économie du Saguenay_Lac-St-Jean. Il n'est peut-être pas inutile de rappeler ici que l'Alcan contrôle l'entière production de l'aluminium primaire de la région, ainsi que la majeure partie de la production hydroélectrique régionale. Il est incontestable que ce sont là deux secteurs clés de l'économie saguenayenne. Par ailleurs, la nature même de l'industrie d'aluminium fait que « la structure la plus économique pour une firme opérant dans ce secteur requiert le contrôle des divers stades de la production »195. En effet, les avantages généraux d'une production intégrée, comme la possibilité d'échelle, l'élimination des gaspillages entre les divers stades de la production et la facilité d'application de méthodes efficaces de fabrication et de gestion, prennent encore plus d'importance dans le cas de l'aluminium. Il est vital pour tout producteur de ce métal de posséder ses propres sources d'énergie et l'on sait que la qualité de l'aluminium dépend en grande partie du type de bauxite utilisée et de la pureté de l'oxyde d'aluminium.

Toutes ces considérations d'ordre technique et de rentabilité économique expliquent comment les sociétés opérant dans ce secteur en sont venues à se tailler de véritables positions de quasi monopole, liées à leur dimension et à leur puissance économique. Il est banal de dire qu'il existe « une certaine relation entre concentration et puissance économique » et que « le comportement de la firme sur son marché est en partie fonction de sa puissance économique » 196. Or, précisément, le « pouvoir de monopole » n'est rien d'autre que « le jeu combiné de la puissance économique et du comportement de la firme sur son marché » 197.

194 F. Perroux, op. cit., pp. 35 et 85.

195 D.H. Wallace, op. cit., p. 202.

196 B. Bonin, L’investissement étranger à long terme au Canada, Presses des HEC, Montréal, 1967, p. 267.

197 J. Houssiaux, Le pouvoir de monopole, Sirey, 1958, p. 64. 
L'effet de domination qu'exerce l'Alcan sur l'économie saguenayenne n'est sans doute « ni général ni total» au sens que F. Perroux donne à cette expression198. Mais, force est de constater, sur un plan plus large, que la Compagnie n'a pas de réels concurrents dans la région et qu'une véritable industrialisation en aval de l'aluminium ne s'y est pas produite. Entre ces deux ordres de faits, il semble qu'il y ait plus qu'un simple rapport de coïncidence.

L'Alcan n'est certes pas au Saguenay-Lac-St-Jean ce que les grandes compagnies internationales de pétrole, par exemple, sont au Moyen-Orient. D'une part, à l'exception de l'énergie hydroélectrique, la région n'offre aucune des matières premières de l'aluminium ; d'autre part, l'Alcan est une compagnie canadienne, dont le siège social est à Montréal. Il n'en reste pas moins qu'en tant que grande unité inter-territoriale l'Alcan a « un plan global d'action à long terme auquel doivent s'intégrer et se subordonner toutes les affaires nationales ou régionales » 199.

L'histoire de l'implantation de l'Alcan au Saguenay_Lac-St-Jean illustre bien comment une firme arrive à bout de ses concurrents potentiels par un pouvoir supérieur de négociation 200. Ce premier obstacle éliminé, la suprématie de la Compagnie était ainsi assurée. En effet, comme le souligne H.D. Wallace, « a complicated process requiring an investment of several millions, limited natural resources, vigorous expansion by the existing firm, uncertainties of obsolescence, desinclination of bankers to provide financial assistance, some or all of these elements have raised effective barriers to entry of a sort with which the antitrust laws as interpreted and administered were not designed to cope » 201.

En ce qui concerne l'absence d'importantes entreprises en aval de l'aluminium au Saguenay_Lac-St-Jean, l'explication semble devoir être moins simple. Il est évident que la Compagnie aurait pu également développer dans la région un secteur de transformation de l'aluminium primaire. On a souligné ailleurs les facteurs qui, d'un strict point de vue de

198 F. Perroux, L'économie du XXe siècle, op. cit., p. 87.

199 Litvak et Maule, «The Multinational Firm and Conflicting National Interests », Journal of World Trade Law, No 3, p. 318.

200 Voir plus haut « L'implantation ».

201 D.H. Wallace, op. cit., p. 152. 
rentabilité économique, ne l'ont pas encouragée en ce sens. Mais, d'un autre côté, une telle politique n'aurait fait que renforcer sa puissance économique et son pouvoir de monopole à l'égard de l'économie régionale. Or, on peut dire, mis à part les obstacles inhérents au milieu lui-même, que la crainte de devoir dépendre d'un monopole pour leur approvisionnement en lingots d'aluminium et en énergie hydroélectrique a pu dissuader nombre d'investisseurs potentiels à s'installer dans la région. Cette importante caractéristique de l'industrie d'aluminium a été mise en nette lumière par l'auteur précité qui souligne : « ... rolling aluminum sheet is a simple operation that any brass or copper mill could easily undertake. Explanation of the hesitancy of independent enterprises to enter this field must be found in a distate for dependence upon monopoly for ingot supply and en the power of integrated monopoly » 202. Ainsi, le milieu n'est pas seul en cause dans ce qui a été déjà souligné de la faiblesse des effets de polarisation technique de l'Alcan.

\subsubsection{Les défauts structurels et les déséquilibres} conjoncturels de l'économie du SLSJ

Il ne semble plus nécessaire de s'étendre sur cette caractéristique de l'économie saguenayenne qui a été largement analysé tout au long de cette étude 203. Cependant, il convient de souligner que l'implantation de l'Alcan au sein de l'économie du Saguenay-LacSt-Jean a entraîné une certaine aggravation des défauts structurels de cette économie. Il est évident que, par la dimension de ses activités, la très forte intensité capitalistique de l'industrie de l'aluminium, son haut degré de productivité et le niveau supérieur des salaires offerts, l'Alcan au Saguenay-Lac-St-Jean fait figure de géant dans un pays de nains. Dans le contexte de libéralisme traditionnel du Québec et du Canada, il ne semble pas sommaire de souligner que l'asymétrie générale présentée par l'Alcan face aux autres unités régionales de production a pu constituer un handicap pour la croissance des autres secteurs de l'économie du Saguenay-LacSt-Jean. Il est certes évident que le personnel au service de l'Alcan forme « l'aristocratie de la main-d’œuvre régionale ». Le prestige de la Compagnie, le niveau élevé des salaires qu'elle peut offrir à ses employés et nombre d'autres facteurs attirent une grande partie des ressources productives. L'Alcan certes n'y peut rien et il est même possible que son comportement de

202 D.H. Wallace, op.cit., p. 152.

203 Voir notamment « Les structures économiques ». 
grande firme ne fait qu'indiquer la voie à suivre aux autres établissements de la région. Mais, en attendant que se produise ce rééquilibre par le haut, il n'en demeure pas moins que les petites et moyennes entreprises régionales se trouvent défavorisées, privées d'une partie des facteurs nécessaires à leur propre croissance. On voit comment le chemin du développement, comme « séquence de déséquilibres », pour reprendre l'expression du Professeur Hirschman, peut être difficile et aléatoire.

D'un autre côté, l'influence dominante exercée par la Compagnie sur l'économie régionale y introduit un élément majeur d'incertitude. De manière explicite, l'Alcan, véritable baromètre économique du Saguenay_Lac-St-Jean, y répercute les moindres variations de la conjoncture mondiale. On a analysé ailleurs deux exemples majeurs de ce type d'action de la Société sur l'économie régionale : le premier, au cours de la grande dépression des années 1930 et le second, pendant la deuxième guerre mondiale.

Compte tenu du poids énorme de l'Alcan dans l'économie régionale, ces fluctuations entraînent d'importants mouvements de main-d'œuvre, les mises à pied brutales constituant le revers de cette médaille. Or, en matière de croissance économique, la continuité du progrès dans la stabilité vaut autant, sinon davantage, que le rythme même de ce progrès. Les déséquilibres conjoncturels que l'Alcan suscite au sein de l'économie saguenayenne et sur lesquels ni elle ni les autorités économiques régionales n'ont et ne peuvent avoir réellement de prise constituent un handicap certain à la mise en oeuvre d'une politique de développement économique de la région. 


\section{CONCLUSION}

$\underline{\text { Retour à la table des matières }}$

Au terme de cette recherche, il importe de dégager le rôle de l'Alcan dans la dynamique de l'évolution de l'économie du Saguenay_Lac-St-Jeanæ. Auparavant, on présentera de façon systématique les éléments théoriques de base qui ont constitué la trame de l'analyse.

Au cours de cette étude, on a essayé d'analyser les effets de l'Alcan sur l'économie saguenayenne, à la lumière de la théorie du développement régional polarisé. Dans cette optique, on a montré que l'action d'une unité de production sur son environnement, s'exerçant par le biais de «ses flux, de ses prix et de ses anticipations », dépend largement de sa dimension propre. Mais, cette action est d'autant plus intense et durable que les liens unissant cette unité au reste de l'économie sont nombreux et solides. Dès lors, quelle que soit l'unité considérée, son influence dépend surtout des caractéristiques du milieu d'implantation et de la nature des relations qu'elle entretient avec lui.

Dans cette perspective, on a établi une distinction relativement sommaire entre la région développée et la région sous-développée. Le «dynamisme d'encadrement » défini comme « une combinaison des trois dynamismes de la croissance (population, innovations et institutions) » 204 est certes différent selon que l'unité de production est implantée dans l'une ou l'autre de ces régions.

204 F. Perroux, « La contribution analytique de la région », dans L'espace et les pôles de croissance, op. cit., p. 70. 
La région développée a été définie comme celle qui permet l'utilisation rationnelle et sur grande échelle de toutes les ressources productives disponibles. Elle fournit de larges possibilités d'économies externes. Ses échanges avec l'extérieur sont importants, mais la majeure partie de l'activité économique se déroule entre les diverses branches et les divers secteurs de l'économie régionale. Véritablement intégré, l'ensemble économique de la région développée constitue le lieu privilégié des mécanismes d'induction. Toute innovation y met en branle les dispositifs d'entraînement caractéristiques et, en termes d'analyse keynésienne, toute dépense autonome y engendre le maximum d'effets multiplicateurs.

L'implantation d'une importante unité de production dans la région développée entraîne ainsi la naissance et le développement de nombreuses activités complémentaires, provoque l'accroissement numérique et la diversification des industries régionales.

À l'inverse, a-t-on souligné, la région sous-développée présente une structure économique inarticulée et tournée vers l'extérieur. Les flux d'échanges intra-régionaux sont restreints, les possibilités d'économies externes, réduites, les structures sociales peu ouvertes aux innovations. Dans l'hypothèse où une firme importante s'y implante, les effets dans le court terme se traduisent par une élévation du niveau des prix, étant donné la faible élasticité de l'offre de production. La faiblesse des liaisons entre les divers secteurs et branches de l'économie et l'inefficacité des mécanismes de diffusion de la croissance expliquent que l'action exercée par la nouvelle firme, en termes d'effets de complémentarités, est minime. L'étroitesse du marché de la région sous-développée et l'absence d'intégration de l'économie font que la majeure partie des effets de la grande firme se produisent à l'extérieur. À cet égard, on ne peut que souscrire à l'idée de M. Penouil, selon laquelle « dans une économie sous-développée, la juxtaposition d'unités de taille moyenne est, sans doute, plus efficace que la création d'une seule grande unité, en raison de l'exiguïté des marchés et du danger d'absence d'intégration d'une grande unité isolée dans l'économie nationale » 205.

205 M. Penouil, « Pôles de développement en régions sous-développés », op. cit., p. 51. 
L'inégalité de l'action d'une grande firme ou d'une quelconque unité de production en fonction du milieu d'implantation et d'autres facteurs porte à considérer avec une certaine prudence la notion générale de développement polarisé. Des degrés sont discernables dans l'action exercée par une entreprise sur une économie. Suivant l'ampleur de cette action, une activité de production peut être simplement motrice, pô1e de croissance ou pôle de développement.

L'activité motrice 206 exerce une action directe sur le taux d'accroissement du produit réel global de la région, provoque des effets indirects, comme l'amélioration des infrastructures, propres à stimuler l'industrialisation de la région. Mais, on ne peut pas encore parler de polarisation, car l'activité motrice est incapable d'entraîner la création d'activités complémentaires, liées à elle, techniquement et économiquement. Cela est le propre du pôle de croissance, complexe industriel qui, souvent, s'édifie autour d'une activité motrice, et dont la moindre initiative déclenche des réactions en chaîne, favorables à la diffusion de la. croissance dans l'espace considéré. Lorsque, de surcroît, à ces effets du pôle de croissance s'ajoutent une mutation profonde des structures socioéconomiques et une modification significative des mentalités et des genres de vie, on dit qu'il s'agit d'un pôle de développement.

À cette classification fondamentale des types d'action que peut exercer une firme sur son milieu d'implantation se greffent des typologies secondaires, dépendant de l'étendue de l'espace géographique ou de l'espace économique où opère le complexe moteur. Si l'espace géographique coïncidant avec la zone d'influence de la firme est restreint, il s'agit d'un point de croissance. Le complexe industriel, dont l'action s'exerce sur un espace géographique étendu, engendre une zone de croissance qui se transforme en axe de croissance en la présence, au sein de cet espace, d'une voie de transport privilégiée le long de laquelle s'implantent l'ensemble des activités économiques. Suivant l'étendue de l'espace économique d'une firme, défini comme étant le réseau de flux réels et financiers émanant d'elle ou convergeant vers elle, l'influence de cette firme peut être régionale, nationale ou internationale.

206 Idem, p. 42 et ss. 
Cette rétrospective sommaire, enrichie au passage, de quelques précisions terminologiques, jette une nouvelle lumière sur le rôle joué par l'Alcan dans l'évolution de l'économie du Saguenay-Lac-St-Jean. On a d'abord tenté de définir la région et d'en présenter les caractéristiques fondamentales avant l'implantation de la Compagnie. La faible industrialisation de cette région, l'étroitesse des marchés internes et le caractère largement primaire de l'économie sont les facteurs principaux qui ont fait considérer l'économie du Saguenay_Lac-St-Jean comme une économie de ressources, située à mi-chemin entre l'économie sous-développée et l'économie développée. Cependant, l'immensité des richesses hydroélectriques de la région, alliée aux facilités naturelles de transport qu'offre une voie d'eau, la rivière Saguenay, devait constituer des contrepoids décisifs pour l'implantation de l'industrie de l'aluminium et l'avenir économique de la région.

On a montré comment le paysage économique régional a en effet complètement changé sous la dynamique impulsion du plus vaste complexe d'aluminium du monde. La présence de l'Alcan, dont la seule usine d'Arvida fournit de l'emploi à plus de 6000 personnes, a suscité la concentration des activités industrielles et tertiaires dans ce qui est devenu la « Conurbation du Haut-Saguenay ». Le contraste naturel entre les comtés agricoles du Lac St-Jean et le comté industriel de Chicoutimi s'est ainsi accentué. Ce processus, qui s'est effectué sur une période relativement longue, a été accompagné d'un développement sans précédent des infrastructures de la région. En témoignent aujourd'hui la moderne ville d'Arvida, création de l'Alcan, les aménagements portuaires de Port-Alfred, les installations et barrages hydroélectriques qui ont fait du Saguenay-Lac-St-Jean l'une des premières régions productrices d'électricité du Canada. Ces réalisations, qualifiées d'effets indirects de l'Alcan, ont constitué de puissants stimulants à la croissance économique générale de la région.

Avec le temps, l'assiette économique de la Société s'étant élargie, ses effets directs sur la région se sont accrus. On a montré, à cet égard, la dépendance dans laquelle se trouve le Saguenay - Lac-St-Jean face aux entreprises de l'Alcan, soit au niveau des emplois soit dans le domaine des revenus et distribués. Si les effets multiplicateurs n'ont pas pu être comptabilisés, ils n'en demeurent pas moins réels et importants. On a tenté de dégager, à cet 
effet, les rapports entre la croissance des activités de l'Alcan et le développement du secteur tertiaire, ainsi que l'urbanisation progressive de la région.

On a cherché pourtant en vain les activités complémentaires, puissantes, que le complexe électro-métallurgique de l'Alcan aurait pu entraîner dans son sillage. Près d'un demi-siècle d'activité de la Compagnie dans la région aurait dû, sans doute, suffire au déclenchement de ces effets sans lesquels on ne peut pas parler réellement de croissance polarisée. À la vérité, les facteurs qui sont allés à l'encontre de la naissance de pareils effets ont été mis en lumière. L'inarticulation des structures économiques régionales, le manque d'ouverture des structures sociales et des mentalités aux innovations et l'exiguïté des marchés locaux, tels ont paru les obstacles majeurs, inhérents au milieu lui-même. Mais, on a souligné également que le caractère de grande unité inter-territoriale de l'Alcan, mieux ancrée aux grands marchés internationaux qu'à l'espace économique saguenayen, n'a pas favorisé la création de ces complémentarités. Or, on sait que «la notion d'intégration est une sorte de complément spatial à la notion de croissance » et que « l'évolution économique harmonieuse suppose un espace intégré » 207. On n'est donc pas surpris que l'énorme «éléphant gris » qu'est l'industrie saguenayenne d'aluminium ait été relativement stérile, son action permissive étant bloquée par son extrapolation et son pouvoir de monopole.

Pourtant, on ne saurait considérer l'Alcan comme une enclave au sein de l'économie du Saguenay_Lac-St-Jean. Certes, on a montré comment les commandes de la Compagnie, notamment en services, entretiennent nombre de petites entreprises de la région. De même, quelques entreprises, modestes il est vrai, consommatrices d'aluminium, ont vu le jour, vivant dans la mouvance de l'Alcan. Cette polarisation, même faible, ajoutée à l'ensemble des effets directs et indirects de la Société, démontre incontestablement le rôle moteur de l'Alcan dans la croissance économique de la région. Mais, dans la mesure où les échanges de l'Alcan avec l'extérieur sont, de loin, plus importants que ceux entretenus avec la région et que la majeure partie de ses effets d'entraînement se produisent au-dehors, le Saguenay_Lac-St-Jean ne constitue pas un véritable espace polarisé, entendu comme «un ensemble de pôles

207 G. Paquet, « Débats sur les orientations du développement économique régional dans la province de Québec », dans L'Actualité économique, avril-juin 1971, p. 125. 
économiques ou d'unités qui entretiennent avec un pôle d'ordre immédiatement supérieur plus d'échanges ou de connexions avec tout autre pôle de même ordre »208.

Dans cette perspective, l'élargissement du marché extérieur de l'aluminium primaire, en dépit de tous les avantages qu'il pourrait procurer à la région, s'avère secondaire. Le problème majeur consiste dans l'intégration de l'entreprise motrice que représente l'Alcan à l'espace économique saguenayen, pour que de pôle potentiel elle devienne un pôle effectif. Certes, compte tenu de la dimension des flux de la Société, on ne saurait s'attendre à ce que ses débouchés en viennent à se situer uniquement ou même principalement à l'échelon du Saguenay-Lac-St-Jean. Mais, la quasi insularité de l'Alcan a duré trop longtemps pour que la priorité soit aujourd'hui accordée au remodelage des structures de l'économie saguenayenne. Il s'agit de mettre en place de solides unités de production qui serviront de relais à la propagation des effets de croissance de cet énorme pôle potentiel. Certes, « la théorie de la polarisation est une théorie conditionnelle de la croissance régionale » 209. Cet effort commun de l'Alcan et de la collectivité régionale, notamment des autorités économiques, est aujourd'hui, non seulement possible, mais nécessaire, dans un Saguenay-Lac-St-Jean peut-être déprimé mais plus sous-développé.

208 J. Boudeville, L’espace et les pôles de croissance, op. cit., p. 27.

209 J. Paelinck, La théorie du développement régional polarisé, Cahiers de l’ISEA, op. cit., p. 47. 


\section{BIBLIOGRAPHIE}

$\underline{\text { Retour à la table des matières }}$

\section{Ouvrages}

ADELMAN, I., Theories of Economic Growth and Development, Standford, 1961.

BAIROCH, P., Révolution industrielle et sous-développement, Société d'Édition d'Enseignement Supérieur, Paris, 1964.

BARAN, P., Économie politique de la croissance, traduction française de L. Mozere, Coll. Économie et Socialisme, Maspero, 1967.

BARRE, R., Économie politique, 2 tomes, Thémis, PUF, 1965.

BEDARD, R., (en collaboration), L'essor économique du Québec, Librairie Beauchemin, Montréal, 1969.

BELL, G., L'énergie et le développement. L'exemple du projet de pôle électro-métallurgique guinéen, Thèse, Grenoble, 1962, Publiée aux Cahiers de l'ISEA, Paris, 1964.

BLANCHARD, Raoul, Le Canada français, PUF, Paris, 1964.

BLANCHARD, Raoul, L'Est du Canada français, Province de Québec, Publication de l'Institut Scientifique Franco-Canadien, Librairie Beauchemin-Montréal et Masson-Paris, 1935.

BONIN, B., L'investissement étranger à long terme au Canada, Presses des Hautes Études Commerciales, Montréal,1967. 
BOUCHARD, Louis-Marie, La conurbation du Saguenay (Québec), Thèse, Université de Strasbourg, 1971.

BOUDEVILLE, J.R., Les espaces économiques, Coll. Que sais- je ?, no 950, PUF, Paris, 1961.

BOUDEVILLE, J.R., Les programmes économiques, Coll. Que sais-je ?, no 1073, PUF, PARIS, 1963.

BOUDEVILLE, J.R., (En collaboration), L'espace et les pôles de croissance, PUF, Paris, 1965.

BYÉ, M., Relations économiques internationales, 2e édition, Dalloz, Paris, 1965.

BYÉ, M., Croissance nationale et économie internationale, Les Cours de Droit, Paris, 19561957.

BYÉ, M., Les principes de la spécialisation internationale, Les Cours de Droit, Paris, 19531954.

CAVES et HOLTON, The Canadian Economy. Prospect and Retrospect, Harvard University Press, 1959.

CHAMBERLIN, E.H., La théorie de la concurrence monopolistique, traduction française de G. Trancart, PUF, Paris, 1953.

CLAVAL, P., Régions. Nations et grands espaces, Éd. M. Th. Génin, Paris, 1968.

CLARK, C., Les conditions du progrès économique, traduction française de A. Morin-Lambert, PUF, Paris, 1960.

COMEAU, R., (en collaboration), Économie québécoise, Cahiers de l'Université du Québec, 1969.

DOBB, M., Croissance économique et sous-développement, traduction française de R. Albeck, Maspero, Paris, 1965

ERBES, R., Essai sur l'intégration économique. De la théorie des espaces économiques à la théorie de l'intégration, Thèse, Paris, 1964.

FREYSSINET, J., Le concept du sous-développement, Mouton \& Cie, Paris-La Haye, 1966.

GADEAU, R., L'aluminium, Édition. A.Colin, Paris, 1958.

GENDARME, R., La pauvreté des nations, Édition. Cujas, Paris, 1963. 
HARVEY, J., La navigation sur le Saguenay, Thèse M.A., Université Laval, Québec, 1963.

HIGGINS, MARTIN et RAYNAULD, Les orientations du développement économique régional dans la province de Québec, Ministère de l'Expansion Économique Régionale, Ottawa, 1970.

HIRSCH, R.D., Les origines et la nature des déséquilibres régionaux du Québec, Planification du Développement Régional, série II, Cahier II/2, COÉQ, Québec, 1967.

HIRSCHMAN, A.O., La stratégie du développement économique, traduction française de F. Pons, Éditions Ouvrières, Paris, 1964.

HOUSSIAUX, J., Le pouvoir de monopole, Sirey, Paris, 1958.

HOWLAND, R.D., Certains aspects régionaux du développement économique du Canada, Commission Royale d'Enquête, Ottawa, 1957.

ISARD, W. et CUMBERLAND, J.H. (Éd.), Planification économique régionale. Techniques d'analyse applicables aux régions sous-développées, publié par l'Agence de Productivité de l'Organisation Européenne de Coopération Économique, Paris, 1961.

ISARD, W. et autres, Methods of Regional Analysis, an Introduction to Regional Science, The Technology Press of the Mass. Institute of Technology et John Wiley \& Sons, Inc., Londres, New-York, 1960.

JOUANDET-BERNADAT, R., Comptes économiques et espaces régionaux, Gautier-Villars, Paris, 1964.

KEYNES, J.M., La théorie générale de l'emploi, de l'intérêt et de la monnaie, traduction française de J. de Largentaye, Payot, Paris, 1955.

KRUEGER, R.R., (Éd.) : (en collaboration), Regional Resource Planning in Canada, Edition révisée, Holt, Rinehart and Winston of Canada Ltd., Montréal-Toronto, 1970.

LAMBERT, D., Les inflations sud-américaines, Institut des Hautes Études d'Amérique Latine, Paris, 1959

LEBEL, G., Horizon 1980, Une étude sur l'évolution de l'économie du Québec de 1946 à 1968 et sur ses perspectives d'avenir, Gouvernement du Québec, MIC, Québec, 1970.

LEBRET, J., Dynamique concrète du développement, Les Éditions ouvrières, Paris, 1963. 
LEWIS, W.A., The Theory of Economic Growth, Homewood, 1955.

MIGUÉ, J.L., (En collaboration), Le Québec d'aujourd'hui. Regards d'universitaires, HMH Hurtubise Ltée, Montréal, 1971.

MONIER, R., Région et économie régionale, Coll. L'Administration Nouvelle, Éd. BergerLevrault, Paris, 1965.

MORAN, P., L'analyse spatiale en science économique, Éd. Cujas, Paris, 1966.

NEMETH, F., L'exode rural et les impératifs de l'aménagement du territoire au Lac St-Jean, Thèse M.A., Université de Montréal, 1968.

PAELINCK, J., DAVIN, L. et DEGEER, L., Dynamique économique de la région liégeoise, Éd. de l'ASLB, Grand Liège, 1959.

PÉPIN, Pierre-Yves, Le royaume du Saguenay en 1968, Ministère de l'Expansion Économique Régionale, Ottawa, 1969.

PERROUX, F., L'économie du XXe siècle, 2e éd., PUF, Paris, 1964.

PERROUX, F., (en collaboration), L'univers économique et social, tome IX de l'Encyclopédie française, Librairie Larousse, Paris, 1960.

PITAVAL, R., Histoire de l'aluminium, métal de la victoire, Publications minières et métallurgiques, Paris, 1946.

RAYNAULD, A., Croissance et structure économique de la Province du Québec, M.I.C., Québec, 1961.

ROSTOW, W.W., Les étapes de la croissance économique, Éd. du Seuil, Paris, 1962.

RYAN, W.F., The Clergy and Economic Growth in Quebec, Presses de l'Université Laval, Québec, 1966.

SCHUMPETER, J., La théorie de l'évolution économique, Paris, 1935.

SMITH, A., Recherches sur la nature et les causes de la richesse des nations, Paris, 1843.

TAYLOR, N.W., A Study of French Canadians as Industrial Entrepreneurs, Thèse, Yale, 1957. 
WALLACE, D.H., Market Control in the Aluminum Industry, Cambridge University Press, 1937.

WEBER, A., Über den Standort der Industrien, Tübingen, 1909.

WONNACOT, R.J., Canadian American Dependence, North Holland Publishing Co., Amsterdam, 1961.

\section{Articles, rapports, divers}

ALCAN : Les précis Alcan.

Idem, Les rapports annuels.

Idem, Publications diverses.

ANGERS, François-Albert, Perspectives économiques de la région du Saguenay-Lac-StJean, Rapport préparé pour le COÉQ, 1952.

AYDALOT, P., Étude sur le processus de polarisation et sur les réactions des industries anciennes à la lumière de l'expérience de Lacq, Cahiers de l'ISEA, L-15, Mars 1965.

AYDALOT, P., Notes sur les économies externes et quelques notions connexes, Revue Économique, no 4, juillet 1965.

BARRE, R., Le développement économique. Analyse et politique, Cahiers de l'ISEA, F-11, avril 1958.

BARRE, R., Les échanges internationaux comme dynamisme de la croissance, Revue d'Économie Politique, mars-avril 1958.

BENOIST, E., « Le trust canadien de l'aluminium voudra-t-il servir le Canada ? », Journal Le Devoir, Montréal, 11 juin 1946.

B.I.T., « Les objectifs d'emploi dans le développement économique », in Études et Documents, Nouvelles Séries, no 62, Genève, 1961.

BLARDONE, G., «La politique de la croissance équilibrée et harmonisée dans le cadre national: pays sous-développés », in L'univers économique et social, t. IX de l'Encyclopédie française, Librairie Larousse, Paris, 1960. 
BOREL, P., «Quelques réflexions sur les modèles de développement », in Développement et Civilisations, no 6, avril-juin 1961.

BOUDEVILLE,J.R., «Croissance économique des pays insuffisamment mis en valeur et peu peuplés », in Les Cahiers économiques, L'observation économique, sociale et financière, juin 1956.

BOUDEVILLE,J.R., Contribution à l'étude des pôles de croissance brésiliens : une industrie motrice, la sidérurgie du Minas Gerais, Cahiers de l'ISEA, F-10, juin 1957.

BOUDEVILLE,J.R., L'économie régionale, espace opérationnel, in Cahiers de l'ISEA, L-3, juin 1958.

BOUDEVILLE,J.R., L'intégration des espaces économiques, in Cahiers de l'ISEA, L-14, septembre 1964.

BROCHU, M., «Les grands objectifs de la navigation d'hiver au Québec », in L'Actualité économique, Montréal, juin-sept. 1967

BROUILLETTE, Benoît, « L'aluminium au Saguenay », in L'Actualité économique, Montréal, octobre 1946.

BROUILLETTE, B., « L'habitat et la population au Saguenay-Lac-St-Jean », in L'Actualité économique, Montréal, janvier 1948.

BROUILLETTE,B. et DAGENAIS,P., « Quelques aspects de l'économie du Saguenay-Lac-StJean, in L'Actualité économique, janv. 1948.

BYÉ, M., La grande unité inter-territoriale dans l'industrie extractive et ses plans, Cahiers de l'ISEA,F-2, sept. 1955.

BYÉ,M., L'autofinancement de la GUI, et les dimensions temporelles de son plan, Revue d'Économie Politique, mai-juin 1957.

BYÉ, M., «La grande unité inter-territoriale », in l'Univers économique et social, t. IX de l'Encyclopédie française, Librairie Larousse, Paris, 1960.

Conseil Économique Régional du Saguenay-Lac St-Jean, Étude économique et financière de la région du Saguenay—Lac-St-Jean, Comité de Finances, Jonquière, 1967-68.

Idem, La forêt - aspect économique et social de la forêt dans la région du Saguenay-Lac-StJean, Comité forestier, août 1967. 
Conseil d’Orientation Économique du Québec, Croissance économique du Québec 1956-1970, Québec, septembre 1965.

DAVUN, L., Phénomènes de polarisation, seuils de croissance et expansion économique en Afrique Centrale, in Cahiers de l'ISEA, V-5, novembre 1962.

DESTANNE de BERNIS, G., «Le rôle du secteur public dans l'industrialisation », in Économie Appliquée, nos 1-2, Paris, 1962.

DUNN, E.S., «Une technique statistique et analytique d'analyse régionale », in Économie Appliquée, octobre-décembre, 1969.

ELLISON, B., « Arvida, a Modern City », in Engineering and Contract Record, july 1945.

ERBES, R., Les trois conditions de l'intégration économique, Revue Économique, no 4, juillet 1965.

FAUCHER, Albert, "Le caractère continental de l'industrialisation au Québec », in Recherches Sociographiques, Vol VI, no 3, Université Laval, Québec, sept.-déc. 1965.

FRUIT, R., «Les effets de croissance d'un pôle sur l'environnement. Analyse du développement du Centre et du Sud du Département du Nord », in Revue Économique, mai 1960.

HARVEY, J., «L'agglomération portuaire de Port-Alfred-Bagotville », Cahiers de Géographie du Québec, no 22, avril 1967.

HOUSSIAUX, J. : Quasi intégration, croissance des firmes et structures industrielles, Revue Économique, no 3, 1957.

INDUSTRIE ET COMMERCE (Ministère), Division du Québec en dix régions et vingt-cinq sous-régions administratives, Bureau de Recherche Économique, Études Régionales, novembre 1966.

Idem, Industrie manufacturière, Région Saguenay_Lac-St-Jean, Publication no 12, décembre 1965.

Idem, La situation actuelle et l'évolution récente de l'industrie manufacturière, Mission de Planification du Saguenay_Lac-St-Jean, tranche industrielle, août 1969.

Idem, Les pôles d'attraction et leurs zones d'influence, Bureau de Recherche Économique, Études Régionales, 1967. 
Idem, Québec, la situation économique, Bureau de Recherche Économique, revue annuelle.

JOUANDET-BERNADAT, R., Notes sur les structures économiques du Saguenay-Lac-StJean, OPDQ, mai 1969.

LE PAS, J., Typologie des économies externes, in Cahiers de l'ISEA, no 6, juin 1968.

LITVAK et MAULE, The Multinational Firm and Conflicting National Interests, Journal of World Trade Law, no 3, mai-juin 1969.

Mc GUIRE et SARRAZIN : Aluminium, histoire de l'Alcan, trad. franç. in Canadian Geographical Journal, 1963.

MISSION DE PLANIFICATION RÉGIONALE SAGUENAY-LAC-ST-JEAN, Esquisse de plan de développement, Rapport synthèse, OPDQ, décembre 1969.

MAILLAT et MATTHEY, Industrialisation et développement régional, Revue Économique et Sociale, Lausanne, juin 1970.

PAELINCK, J., Croissance régionale et mise en valeur des ressources en Amérique Latine, Cahiers de l'ISEA, L-13, oct.1963.

PAELINCK, J., La théorie du développement régional polarisé, in Cahiers de l'ISEA, L-15, mars 1965.

PARENTEAU, Roland, Les problèmes du développement régional dans un État fédératif: l'expérience canadienne, Communication au Congrès des Économistes de Langue Française, mars 1963.

PENOUIL, M., Pôles de développement en régions sous-développées de pays développés et en pays sous-développés, Communication au Colloque du 18 au 23 mai 1969, Genève.

PEPIN, Pierre-Yves, Principaux traits socio-économiques de la région du Saguenay-Lac-St-Jean, Cahiers de Géographie du Québec, Vol. VII, no 13, oct. 1962 - mars 1963.

PERROUX, F., Esquisse d'une théorie de l'économie dominante, Économie Appliquée, Nos 2-3, 1948, reproduite in F. Perroux : L'économie du XXe siècle, PUF, 1964.

Idem, Les espaces économiques, in Économie Appliquée, no 1, 1950, reproduit in F.Perroux : L'économie du XXe siècle, PUF,1964. 
Idem, Note sur le dynamisme de la domination, in Économie Appliquée, no 2, 1950, reproduit in L'économie du XXe siècle.

Idem, Matériaux pour une analyse de la croissance économique, Cahiers de l'ISEA, D-8, avril 1955.

PERROUX, F., « Note sur la notion de pôle de croissance », in Économie Appliquée, nos 1-2, 1955, reproduit in L'Économie du XXe siècle, PUF, 1964.

Idem, La firme motrice dans une région et la région motrice, in Cahiers de l'ISEA, AD-1, mars 1961.

Idem, Les pôles de développement et l'économie internationale, The Challenge of Development, A Symposium, Jérusalem, 1957, reproduit in L'Économie du XXe siècle, PUF, 1964.

RIBET-PETERSEN, CH. De, Ports et pôles : les aspects originaux de la polarisation dans l'Ouest algérien, in Cahiers de l'ISEA, L-16, février 1966.

ROSENFELD, F., Les firmes motrices et la comptabilité régionale, in Cahiers de l'ISEA, L-11, octobre 1962.

SAICANS, A., Éléments d'analyse des principales variables économiques de la région du Saguenay_Lac St-Jean, Office de Planification et de Développement du Québec, août 1969.

ZARKA, C., De la distinction entre la «croissance » et le "développement» dans la littérature scientifique consacrée aux pays sous-développés, Cahiers de l'ISEA, F-12, 1959.

Fin. 